\title{
Synthesis of heterocycles: Indolo[2,1-a]isoquinolines, renewables, and aptamer ligands for cellular imaging \\ by \\ Jonathan Beasley
}

A dissertation submitted to the graduate faculty in partial fulfillment of the requirements for the degree of DOCTOR OF PHILOSOPHY

Major: Organic Chemistry

Program of Study Committee:

George A. Kraus, Major Professor

William S. Jenks

Raj Raman

Klaus Schmidt-Rohr

Yan Zhao

Iowa State University

Ames, Iowa

2013

Copyright (C) Jonathan Beasley, 2013. All rights reserved. 


\section{TABLE OF CONTENTS}

GENERAL INTRODUCTION

CHAPTER 1. A ONE-POT CONVERSION OF ORTHO-ALKYNYL BENZALDEHYDES INTO INDOLO[2,1-a]ISOQUINOLINES

Introduction 2

Results and Discussion $\quad 4$

$\begin{array}{ll}\text { Experimental } & 14\end{array}$

$\begin{array}{ll}\text { References } & 30\end{array}$

CHAPTER 2. BIO-BASED APPROACHES TO MALEIC ACID AND IONIC LIQUIDS

Introduction $\quad 32$

Results and Discussion 34

$\begin{array}{ll}\text { Experimental } & 40\end{array}$

$\begin{array}{ll}\text { References } & 46\end{array}$

CHAPTER 3. REAL-TIME IMAGING OF PROMOTER ACTIVITY TO MONITOR GENE EXPRESSION

$\begin{array}{ll}\text { Introduction } & 48\end{array}$

$\begin{array}{lr}\text { Results and Discussion } & 50\end{array}$

$\begin{array}{lr}\text { Experimental } & 59\end{array}$

$\begin{array}{ll}\text { References } & 69\end{array}$

$\begin{array}{ll}\text { GENERAL CONCLUSION } & 71\end{array}$

$\begin{array}{ll}\text { ACKNOWLEDGEMENTS } & 73\end{array}$ 


\section{GENERAL INTRODUCTION}

The synthesis of biologically active molecules represents an important aspect of organic chemistry. Renewables, and their respective green syntheses, embody another essential aspect of organic chemistry receiving growing interest. Each step toward target molecules, either molecules of biological or renewable interest, should employ optimal reactions from optimal starting materials. Therefore, each reaction step of a synthesis should result in good yield of product with little work.

In this thesis, we explore both total syntheses and methodologies of several aromatic heterocyclic molecules. Chapter 1 describes extensions of the Kraus indole synthesis toward 2substituted and 2,3-disubstituted indoles, as well as biologically attractive indolo[2,1a]isoquinolines. Chapter 2 describes recent renewable efforts directed to commodity maleic acid and the first reported furan-based ionic liquids. Chapter 3 describes our total synthesis of mRNA aptamer ligand PDC-Gly, and its dye coupled forms, plus aminoglycoside dye coupled ligands used in molecular imaging. This work was conducted in collaboration with molecular biologists at Iowa State University. 


\section{CHAPTER 1. A ONE-POT CONVERSION OF ORTHO-ALKYNYL BENZALDEHYDES INTO INDOLO[2,1-a]ISOQUINOLINES}

\section{Introduction}

The indole subunit is present in a wide array of biologically active molecules. ${ }^{1}$ Consequently, many useful synthetic pathways for their syntheses have emerged and been cataloged. ${ }^{2}$ Recently, Taber and Tirunahari published a comprehensive review of indole reactions, categorizing their syntheses based on the last bond formed in the indole subunit into seven types (Figure 1$){ }^{3}$

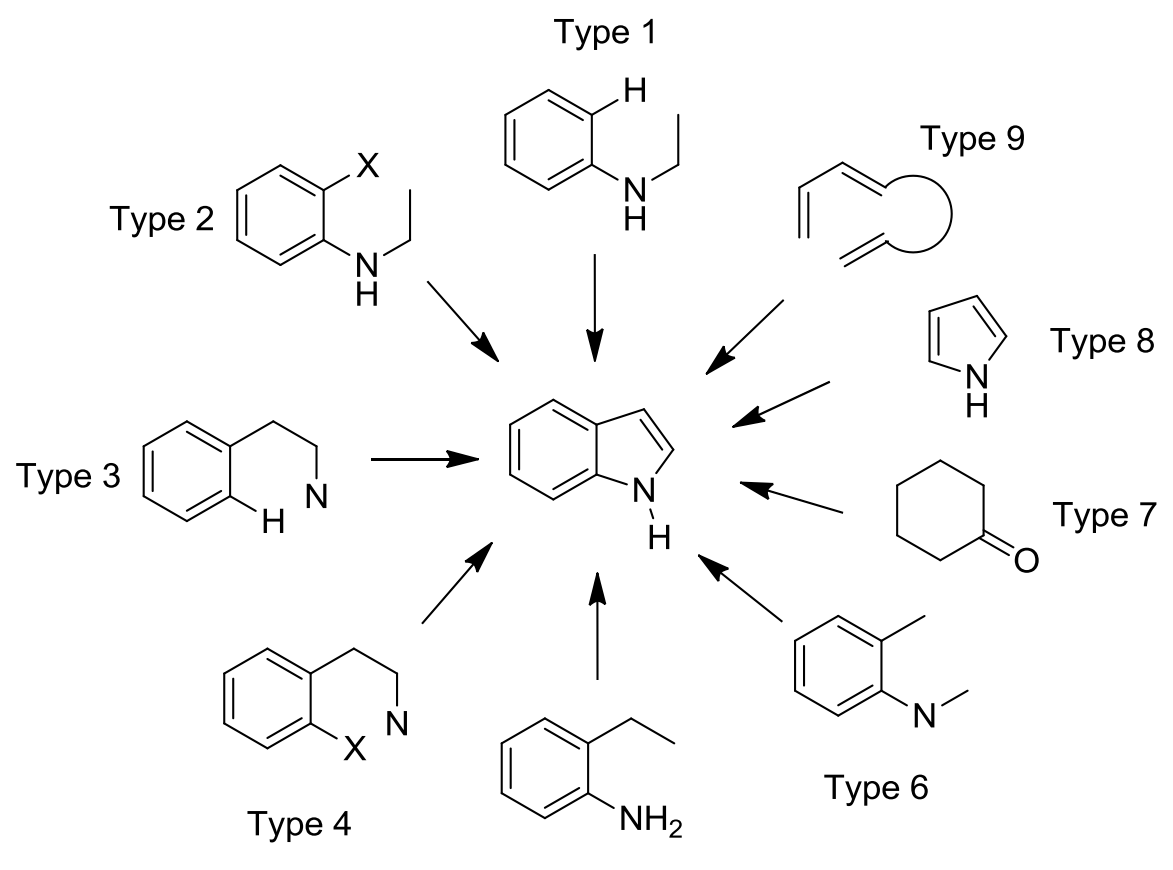

Type 5

Figure 1. Nine types of indole syntheses

For example, type 1 could correspond to the Fischer indole synthesis, ${ }^{4}$ Bartoli indole synthesis, ${ }^{5}$ as well as the Gassman indole synthesis, ${ }^{6}$ where the last bond formed results from electrocyclic ring closure. Hydrazones and anilines are among the most common precursors to 
indoles as seen in the Fischer indole synthesis and Japp-Klingemann indole synthesis. ${ }^{7}{ }^{\text {The }}$ Larock indole synthesis ${ }^{8}$ could be considered a type 5 transformation, where internal alkynes couple with an ortho-haloaniline in the presence of palladium catalyst and cyclize onto the arylamine. Similarly, ortho-haloanilines react with alkenes to generate substituted indoles (Scheme 1). ${ }^{9}$<smiles>[X]c1ccccc1N</smiles>

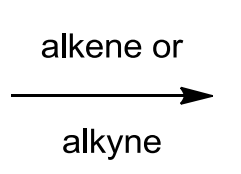<smiles>[R]c1[nH]c2ccccc2c1[R]</smiles>

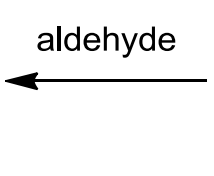<smiles>Nc1ccccc1CCl</smiles>

Scheme 1. Indole synthesis from anilines

ortho-Alkylindoles have been less frequently employed. One example is the Madelung synthesis, in which the dianion of an anilide undergoes cyclization at high temperatures. ${ }^{10}$ Another example is the Bischler-Möhlau indole synthesis, a microwave-assisted method that couples anilines with bromoacetophenones. ${ }^{11}$ Recently, the Kraus group published a novel, flexible, one-pot synthesis of indoles utilizing a type 6 pathway (Scheme 2). ${ }^{12}$<smiles>[R]C=Nc1ccc(C(C)C)cc1CP[Pb](C)(Br)C(C)=O</smiles>
1

Scheme 2. Indole synthesis by Kraus and Guo

In an effort to expand the usefulness of this work, other functional groups were investigated in lieu of the triphenylphosphine group. Further, the flexibility of the Kraus indole synthesis was tested in efforts toward the synthesis of indolo[2,1-a]isoquinolines, a class of 
compounds which exhibit, along with their dihydro analogs, a diverse assortment of biological activities. These compounds are components in tubulin-binding drugs, ${ }^{13}$ estrogen receptor modulators, ${ }^{14}$ and organic semiconductors. ${ }^{15}$ Some members of this class are natural products: (S)-(-)-cryptaustoline (2) and (S)-(-)-cryptowoline (3) (Figure 2). ${ }^{16}$

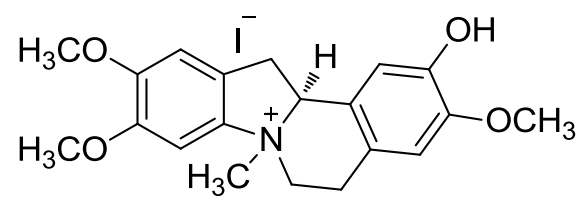

(S)-(-)-cryptaustoline (2)

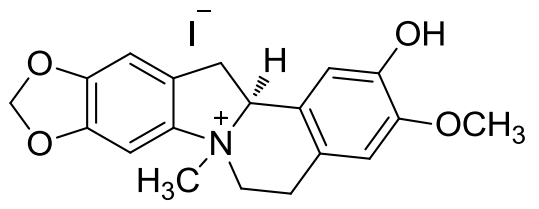

(S)-(-)-cryptowoline (3)

Figure 2. Natural products with indolo[2,1-a]isoquinoline backbones

Because of their biological activity, many approaches to this class of compounds have been reported. The most common approach involves transition metal catalyzed cyclizations such as organopalladium couplings of 2-aryl indoles through both intermolecular ${ }^{17}$ and intramolecular ${ }^{18}$ pathways, copper-mediated cyclizations, ${ }^{19}$ rhodium $^{20}$ and platinum-based cyclizations. $^{21}$ In the process of extending our indole synthesis, we have discovered a novel tandem cyclization to indolo[2,1-a]isoquinolines in the absence of transition metal catalysts.

\section{Results and Discussion}

In further developing the flexibility and breadth of the Kraus indole synthesis, we investigated the effect of sulfides as a group G (Scheme 3). Previous Kraus group members had determined that both triphenylphosphine and arylsulfones were eliminated upon electrocyclic cyclization and aromatization to 2-substituted indoles, while nitrile groups were retained. ${ }^{22}$ My efforts were focused on the effects of sulfides and whether the sulfide group was retained or lost 
during cyclization. Since the desired thioanilines were not commercially available, the project commenced with their synthesis from commercially available starting materials.<smiles>Nc1ccccc1CCl</smiles><smiles>[R]C(C)O[CH]C</smiles><smiles>[R]C=Nc1ccccc1CO</smiles><smiles>CCCCCCC</smiles><smiles>[R]C=[N+]([O-])c1ccccc1CCCC</smiles><smiles>[R]c1[nH]c2ccccc2c1C#N</smiles>

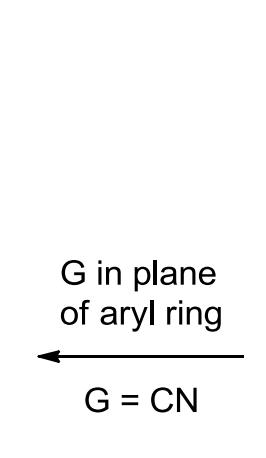<smiles>CI</smiles><smiles>[R]C1Nc2ccccc2C1C</smiles>
$\mathrm{G}$ perpendicular
to aryl ring
$\mathrm{G}=\mathrm{PPh}_{3}{ }^{+}, \mathrm{SO}_{2} \mathrm{Ph}$<smiles>[R]c1cc2ccccc2[nH]1</smiles>

Scheme 3. Proposed Kraus indole synthesis mechanism

To test the effect of sulfides on the Kraus indole synthesis, we initially chose group G to be phenylsulfide, a known leaving group. To generate the desired thioaniline adduct, we invoked known transformation of benzylic alcohols to phenylsulfides (Scheme 4). ${ }^{23}$ The subsequent 2-((phenylthio)methyl)aniline (5) was submitted to standardized Kraus indole conditions to no avail. Reasoning that the benzylic position might have a lower acidity than previously successful systems containing group $\mathrm{G}$ as triphenylphosphine or nitrile, where the 
cyclization was initiated with potassium tert-butoxide, we employed lithium diisopropylamide (LDA) as a stronger base to affect the cyclization. The resulting indole $\mathbf{6}$ was formed in modest yields. Interestingly, the phenylsulfide group proved to be a leaving group during the cyclization step.<smiles>Nc1ccccc1CO</smiles>

4

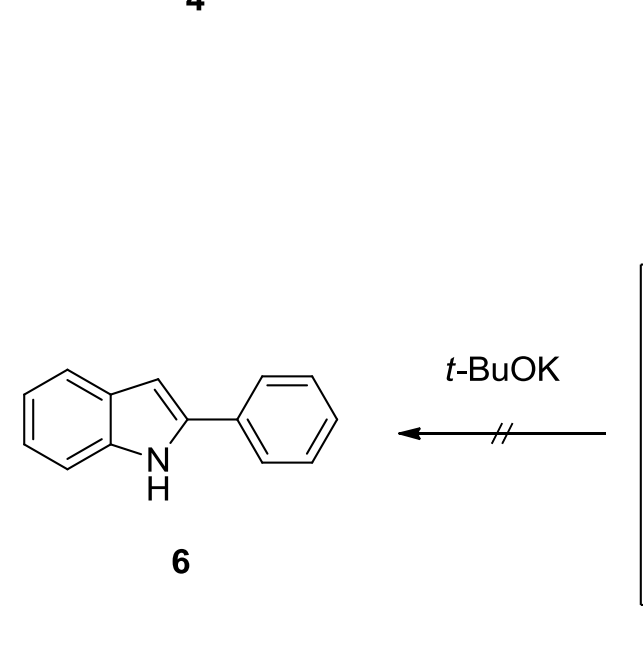

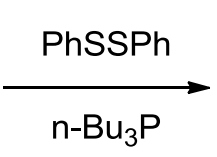

5<smiles>Nc1ccccc1CSc1ccccc1</smiles>

$\mathrm{PhCHO}$, $\mathrm{AcOH}$, microwave

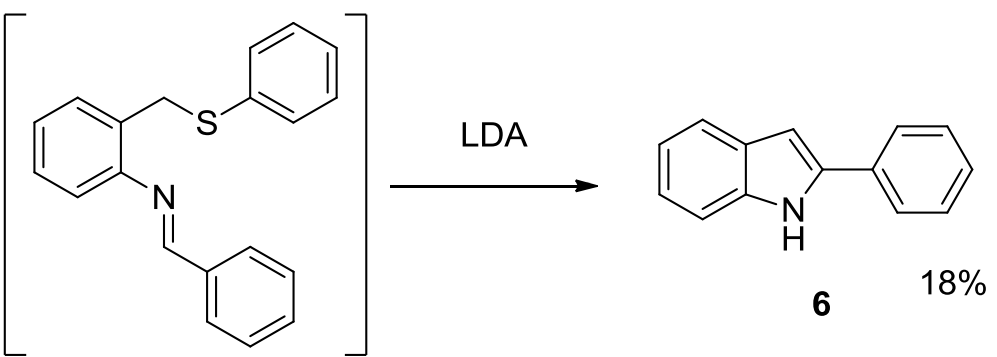

Scheme 4. Synthesis of indole 6

To confirm our result, we set out to synthesize 2-(bis(methylthio)methyl)aniline (7) utilizing a Sommelet-Hauser rearrangement prescribed by Gassman (Scheme 5) ${ }^{24}$ The resulting aniline was subjected to standard Kraus indole conditions to generate 2,3-disubstitued indoles 8 and $\mathbf{9}$ in modest to good yields. In agreement with the phenylsulfide results, we saw loss of one methylsulfide group in aromatization of the indole unit. 


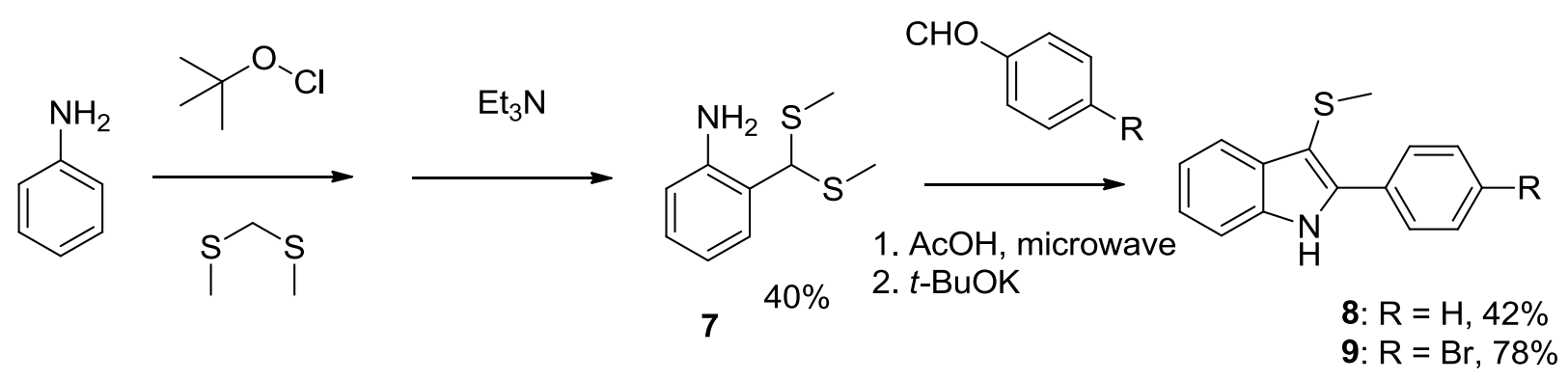

Scheme 5. Synthesis of indoles 8 and 9

To further extend the science of the Kraus indole synthesis, we sought to develop a method to synthesize indolo $[2,1-a]$ isoquinolines from aromatic aldehydes and commercially available 1. The decision to switch from thioethers to $\mathbf{1}$ was two-fold: The phosphonium salts gave higher yields in previous reactions ${ }^{12,22}$ and the phosphonium salt was commercially available while the thioethers were not. With the aniline starting material determined, we focused our attention on developing the aldehyde portion.

The aldehydes used for the Kraus indole reaction were synthesized via Sonogashira coupling of ortho-halobenzaldehydes with terminal alkynes (Table 1). Commercially available 2-bromobenzaldehyde was used directly, while all of the 2-iodobenzaldehydes had to be synthesized from commercially available materials. Commercially available 2-iodobenzyl alcohol was oxidized with manganese(IV) to give 2-iodobenzaldehyde. Electron rich 2-iodo-4,5dimethoxybenzaldehyde was synthesized by electrophilic aromatic substitution with iodine and silver(I) trifluoroacetate. ${ }^{25}$ Lastly, 2-iodo-3-methoxybenzaldehyde was synthesized via Comins $^{26}$ chemistry utilizing ortho-lithiation followed by electrophilic quenching by way of iodine. The Sonogashira coupling was accomplished using standard conditions. Generally, a marked yield increase was seen by changing the halide from bromide to iodide. Sterics also play a role as seen 
in entries 2 and 3 and entries 4 and 11. With compounds 10a-k in hand, we were ready to submit them to standardized Kraus indole synthesis conditions.

Table 1. Alkynyl benzaldehydes from Sonogashira coupling

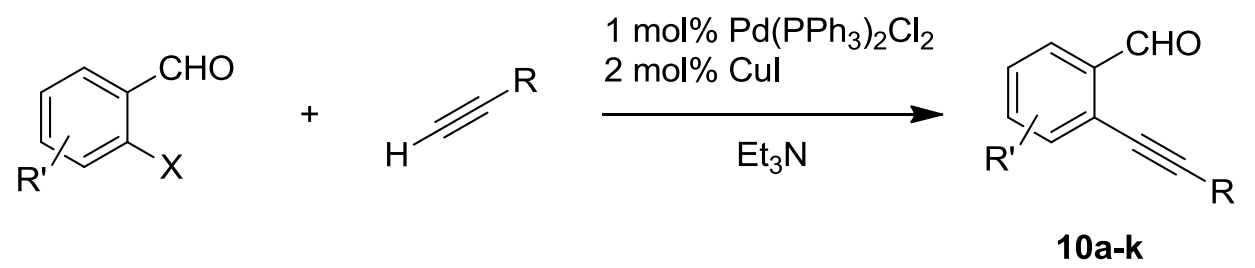

Entry


8<smiles>O=Cc1ccccc1I</smiles>

9<smiles>COc1cc(I)c(C=O)cc1OC</smiles>

10<smiles>O=Cc1cc2c(cc1Br)OCO2</smiles>

11<smiles>COC(C)C#Cc1ccccc1C=O</smiles>

10h $35^{\varepsilon}$<smiles>COCC#Cc1cc(OC)c(OC)cc1C=O</smiles>

$10 \mathrm{i}$

$87^{\gamma}$

10j $39^{\gamma}$<smiles>COCC#Cc1cc2c(cc1C=O)OCO2</smiles>

10k $29^{\beta}$

${ }^{\alpha}$ Isolated yield, not optimized. ${ }^{\beta}$ Reaction ran at $50{ }^{\circ} \mathrm{C} .{ }^{\gamma}$ Reaction ran in THF. ${ }^{\delta}$ Yield based on ${ }^{1}$ H NMR. ${ }^{\varepsilon}$ Alcohol product was unstable. Crude was converted to the methoxymethyl ether. Yield reflects the final yield after the 2-step process.

The first system we tried the indole synthesis on was with benzaldehyde 10a, entry 1 Table 2, which produced 11a in poor yield. Interestingly, the TMS group was also removed during the one-pot reaction, ostensibly due to nucleophilic attack of potassium tert-butoxide on the trimethylsilyl group. Other indoles (11b-e) were prepared in anticipation of cyclizing them to indolo[2,1-a]isoquinolines via Kraus indole conditions (Table 2). 
Table 2. Table of indole products

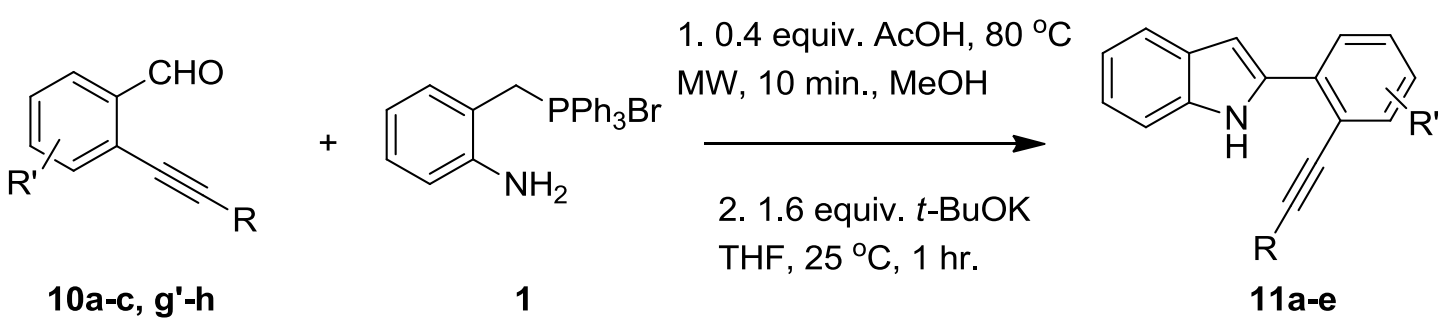

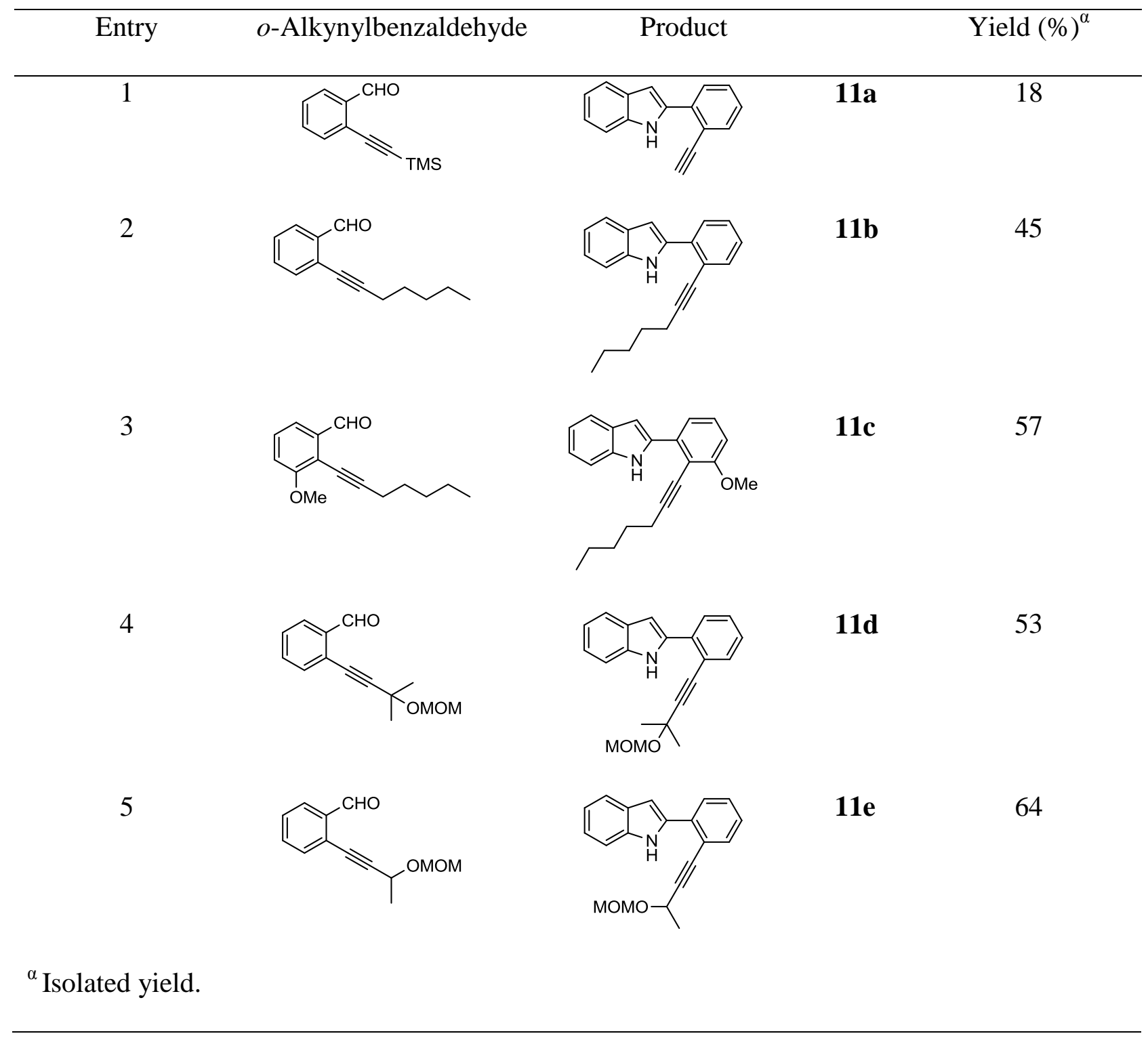

In the midst of synthesizing indoles, we ran across an interesting and desirous result when cyclizing aldehyde 10d. The major organic product lacked the characteristic N-H bond as 
evidenced by ${ }^{1} \mathrm{H}$ NMR in indoles, and the acetylenic resonances were absent in the ${ }^{13} \mathrm{C}$ NMR. Instead of generating the expected indole, indolo[2,1-a]isoquinoline 12a was the only isolable product formed. Building upon this result, other propargyl ethers (10d-f, i-k) were reacted under Kraus indole conditions to generate a small library of indolo[2,1-a]isoquinolines (Table 3). The ${ }^{1} \mathrm{H}$ and ${ }^{13} \mathrm{C}$ NMR data, as well as high resolution mass spectrum, support the assignment of each of the indolo[2,1-a]isoquinolines.

Table 3. Table of indolo[2,1-a]isoquinolines<smiles>[R]C#Cc1c[R]([R])ccc1C=O</smiles>

10d-f, i-k

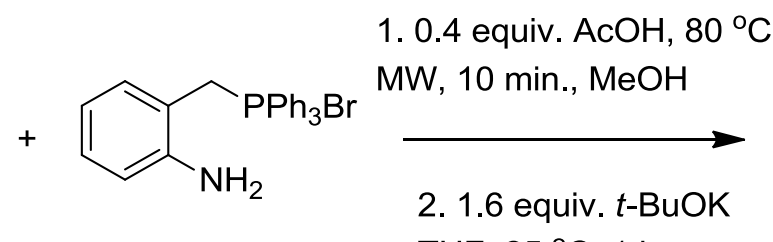

1

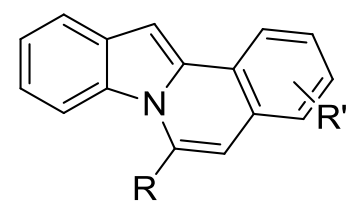

12a-f
Yield $(\%)^{\alpha}$
31<smiles>COCc1cc2ccccc2c2cc3ccccc3n12</smiles><smiles>COCc1cc2ccccc2c2cc3ccccc3n12</smiles>

12b

29<smiles>O=Cc1cc2ccccc2c2cc3ccccc3n12</smiles>

12c

22<smiles>COCc1cc2cc(OC)c(OC)cc2c2cc3ccccc3n12</smiles>

12d

65 
5

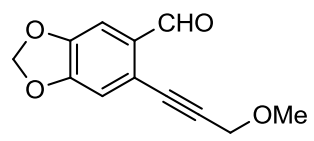

6

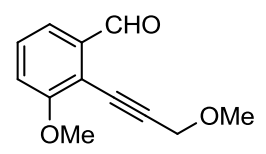

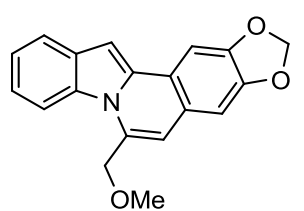

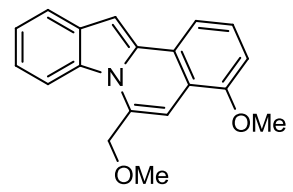

12e

36

$12 f$ 28

${ }^{\alpha}$ Isolated yield.

Entry 3 gave an interesting result as the acetal was also deprotected, an unexpected result of the Kraus indole conditions. It is important to note that the yield of $\mathbf{1 2 c}$ (entry 3), albeit only $22 \%$, is the result of four steps: Imine formation, cyclization to the indole, closure of the alkyne, and hydrolysis. Compounds $\mathbf{1 2 d}$ and $\mathbf{1 2 e}$ (entries 4 and 5) are more representative of natural products in the indolo[2,1-a]isoquinoline family and gave the highest yields.

Presumably, the indolo[2,1-a]isoquinoline products arose from the cyclization of the anion of the indole onto the available alkyne (Scheme 6). Sterics seemingly play a role, as Table 2, entries 4 and 5 indicate sterically encumbered propargyl groups do not cyclize. Further, electronics also play a major role. Table 2, entries $1-3$ show that propargyl groups are necessary to affect the final cyclization to indolo[2,1-a]isoquinolines. We suggest that there may be an equilibrium between the anion of the indole and the vinylic anion produced by cyclization to indolo[2,1-a]isoquinolines and that the methoxymethyl group stabilizes the vinylic anion so that protonation by tert-butanol generates indolo[2,1-a]isoquinolines. 


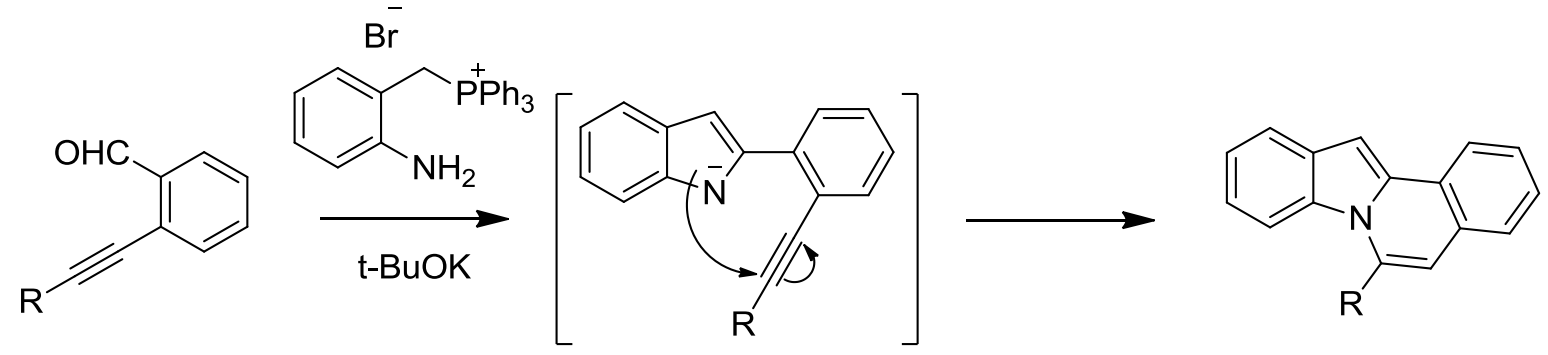

Scheme 6. Proposed indole intermediate to indolo[2,1-a]isoquinolines

\section{Conclusions}

The synthesis of indoles using aminobenzyl sulfides or phosphonium salts is a direct and flexible strategy. The use of sulfides expands the scope and limitations of the Kraus indole synthesis outside of previously established routes utilizing phosphonium salts, arylsulfones, and nitriles. Further, the cyclization and loss of sulfide allows for molecular flexibility that phosphonium salts and nitriles might not offer, while still directing the substitution at the 2- and 3-positions of the formed indole. The Kraus indole cyclization proceeds at room temperature and is compatible with sulfide functional groups.

Further, the conversion of 2-halobenzaldehydes into indolo[2,1-a]isoquinolines by a Sonogashira reaction followed by an indole forming reaction represents a convenient route to this useful class of molecules. Similar to the sulfide series, the indole forming reaction proceeds at room temperature and is compatible with several functional groups. No transition metal catalysts are required while complex ring systems are stitched together in $2-3$ steps. This synthesis expands the scope of the Kraus indole synthesis and offers attractive, new pathways to indolo[2,1-a]isoquinolines. 


\section{Experimental Section}

All NMR spectra were obtained on a Varian VXR spectrometer, operating at 300 or 400

$\mathrm{MHz}$ for ${ }^{1} \mathrm{H}$ NMR and 75 or $100 \mathrm{MHz}$ for ${ }^{13} \mathrm{C}$ NMR instrument. Chemical shifts in $\mathrm{CDCl}_{3}$ were reported downfield from TMS (=0 ppm) for ${ }^{1} \mathrm{H}$ NMR. For ${ }^{13} \mathrm{C}$ NMR, chemical shifts were reported relative to the solvent signal $\left[\mathrm{CDCl}_{3}(77.15 \mathrm{ppm})\right]$. All reactions were carried out under argon unless otherwise noted. Microwave-assisted reactions were performed in a capped vial using a CEM Discover System. Thin-layer chromatography was performed using commercially available 250 micron silica gel plates (Analtech). Preparative thin-layer chromatography was performed using commercially available 1000 micron silica gel plates (Analtech). Visualization of TLC plates was effected with short wavelength ultra violet light $(254 \mathrm{~nm})$. High resolution mass spectra were recorded on an Agilent 6540 QTOF using EI, ESI, or ACPI. All reagents were used directly as obtained commercially unless otherwise noted.

General procedure for the synthesis of 2-alkynyl benzaldehydes. To a solution of 2halobenzaldehyde $(1 \mathrm{mmol})$ and alkyne $(1.2 \mathrm{mmol})$ in triethylamine $(10 \mathrm{~mL})$ was added bis(triphenylphosphine)palladium(II) chloride $(0.01 \mathrm{mmol})$. In cases where the benzaldehyde was insoluble in triethylamine, tetrahydrofuran (THF) was added to help solubilize the aldehyde. After 5 minutes of stirring, copper(I) iodide $(0.02 \mathrm{mmol})$ was added. The reaction was stirred overnight, resulting in a brown suspension. The solid was filtered off through Celite ${ }^{\circledR}$ and the filtrate was concentrated under vacuum. The crude residue was purified by silica gel column chromatography using a mixture of ethyl acetate and hexanes as eluent.

General procedure for the synthesis of 2-substituted indoles. In a $10 \mathrm{~mL}$ microwave reaction vial (CEM Discover System) equipped with a magnetic stir bar, aniline (0.5 mmol), aldehyde $(0.5 \mathrm{mmol})$, and glacial acetic acid $(0.01 \mathrm{~mL}, 0.17 \mathrm{mmol})$ were added to dry methanol 
(3.0 mL). The vial was capped and placed into the microwave, which was then run at 300 Watts, to $80{ }^{\circ} \mathrm{C}$ for 10 minutes. After cooling to room temperature the solution was transferred to a round bottom flask and the methanol was removed under vacuum. Tetrahydrofuran $(10.0 \mathrm{~mL})$ was added to the mixture, followed by a $1 \mathrm{M}$ solution of $\mathrm{t}-\mathrm{BuOK}$ in $\mathrm{THF}(0.8 \mathrm{~mL}, 0.8 \mathrm{mmol})$ slowly at room temperature. The resulting mixture was stirred for 2 hours at room temperature. The reaction was quenched with a saturated solution of $\mathrm{NH}_{4} \mathrm{Cl}$ and extracted three times with ethyl acetate. The combined organic layers were dried over anhydrous $\mathrm{MgSO}_{4}$ and filtered. The filtrate was concentrated under vacuum, and the crude residue was purified by silica gel column chromatography or preparative TLC using a mixture of ethyl acetate and hexanes as eluent.

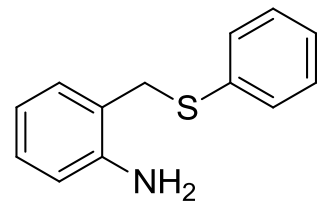

2-((Phenylthio)methyl)aniline $5:^{23}$ To a solution of phenyl disulfide $(1,375 \mathrm{mg}, 6.3$ $\mathrm{mmol})$ and (2-aminophenyl)methanol $(730 \mathrm{mg}, 5.9 \mathrm{mmol})$ in dichloromethane $(40 \mathrm{~mL})$ was added n-tributylphosphine $(1.56 \mathrm{~mL}, 6.3 \mathrm{mmol})$ dropwise over 15 - 20 minutes. The reaction was heated to reflux overnight, and then cooled to room temperature. Water was added and the product was extracted twice with dichloromethane. The combined organic layers were washed with brine, dried over anhydrous $\mathrm{MgSO}_{4}$ and filtered. The residue was purified by silica gel column chromatography using a mixture of ethyl acetate and hexanes as eluent. Yellow solid (243 mg, 19\%). ${ }^{1} \mathrm{H}$ NMR (300 MHz, $\left.\mathrm{CDCl}_{3}\right):$ 7.17-7.36 (m, 5H), $7.08(\mathrm{td}, \mathrm{J}=7.8,1.5 \mathrm{~Hz}, 1 \mathrm{H})$, $6.97(\mathrm{~d}, \mathrm{~J}=7.5 \mathrm{~Hz}, 1 \mathrm{H}), 6.64-6.70(\mathrm{~m}, 2 \mathrm{H}), 4.08(\mathrm{~s}, 2 \mathrm{H}), 4.01(\mathrm{br} \mathrm{s}, 2 \mathrm{H}) .{ }^{13} \mathrm{C} \mathrm{NMR}(75 \mathrm{MHz}$, $\left.\mathrm{CDCl}_{3}\right): 145.1,135.7,130.7,130.3,128.8,128.7,126.6,120.9,118.7,116.4,36.8$. 


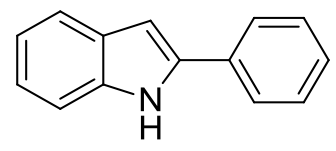

2-Phenyl-1H-indole 6: $\mathrm{In}$ a $10 \mathrm{~mL}$ microwave reaction vial (CEM Discover System) equipped with a magnetic stir bar, aniline $5(0.5 \mathrm{mmol})$, benzaldehyde $(0.5 \mathrm{mmol})$, and glacial acetic acid $(0.01 \mathrm{~mL}, 0.17 \mathrm{mmol})$ were added to dry methanol $(3.0 \mathrm{~mL})$. The vial was capped and placed into the microwave, which was then run at $300 \mathrm{Watts}$, to $80^{\circ} \mathrm{C}$ for 10 minutes. After cooling to room temperature the solution was transferred to a round bottom flask and the methanol was removed under vacuum. Tetrahydrofuran $(10.0 \mathrm{~mL})$ was added to the mixture, which was cooled to $-78{ }^{\circ} \mathrm{C}$. To a solution of recently distilled $\mathrm{N}, \mathrm{N}$-diisopropylamine $(0.03 \mathrm{~mL}$, $0.21 \mathrm{mmol})$ in $\mathrm{THF}(10 \mathrm{~mL})$ at $0{ }^{\circ} \mathrm{C}$ was added a $2.5 \mathrm{M}$ solution of n-butyllithium in hexanes $(0.08 \mathrm{~mL}, 0.2 \mathrm{mmol})$ with stirring. After 10 minutes at $0{ }^{\circ} \mathrm{C}$, the reaction was cooled to $-78{ }^{\circ} \mathrm{C}$, and then added to the crude imine solution dropwise. The resulting brown solution was warmed to $-15{ }^{\circ} \mathrm{C}$ for 30 minutes, then to $0{ }^{\circ} \mathrm{C}$ for 1 hour. The reaction was warmed to room temperature and stirred overnight. The reaction was quenched with saturated $\mathrm{NH}_{4} \mathrm{Cl}$, and extracted three times with ethyl acetate. The combined organic layers were washed with brine, dried over anhydrous $\mathrm{MgSO}_{4}$ and filtered. The filtrate was concentrated under vacuum, and the crude residue was purified by preparative thin-layer chromatography using a mixture of ethyl acetate and hexanes as eluent. Brown solid (8 mg, 18\%). ${ }^{1} \mathrm{H}$ NMR (300 MHz, $\left.\mathrm{CDCl}_{3}\right): 8.34$ (br s, 1H), 7.62-7.70 (m, 3H), $7.44(\mathrm{q}, \mathrm{J}=6.0 \mathrm{~Hz}, 3 \mathrm{H}), 7.33(\mathrm{t}, \mathrm{J}=6.0 \mathrm{~Hz}, 1 \mathrm{H}), 7.20(\mathrm{td}, \mathrm{J}=8.2,1.2 \mathrm{~Hz}$, $1 \mathrm{H}), 7.12(\mathrm{td}, \mathrm{J}=8.2,1.2 \mathrm{~Hz}, 1 \mathrm{H}), 6.84(\mathrm{~d}, \mathrm{~J}=1.2 \mathrm{~Hz}, 1 \mathrm{H}) .{ }^{13} \mathrm{C} \mathrm{NMR}\left(75 \mathrm{MHz}, \mathrm{CDCl}_{3}\right): 137.8$, 136.7, 132.3, 129.2, 129.0, 127.7, 125.1, 122.3, 120.6, 120.2, 117.4, 110.8, 100.0. 


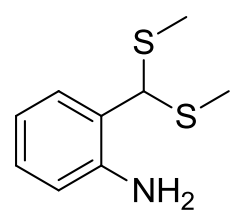

2-(Bis(methylthio)methyl)aniline $7:{ }^{27}$ To a solution of freshly distilled aniline $(0.81$

$\mathrm{mL}, 8.9 \mathrm{mmol})$ and bis(methylthio)methane $(0.93 \mathrm{~mL}, 9.1 \mathrm{mmol})$ in dry dichloromethane $(50$ $\mathrm{mL}$ ) at $-78{ }^{\circ} \mathrm{C}$ was added a solution of tert-butyl hypochlorite $(990 \mathrm{mg}, 9.1 \mathrm{mmol})$ in dichloromethane $(10 \mathrm{~mL})$ dropwise with stirring. The reaction solution was allowed to stir for 1 hour at $-78^{\circ} \mathrm{C}$, and then triethylamine $(5.0 \mathrm{~mL}, 36 \mathrm{mmol})$ was added. The reaction solution was warmed to $0{ }^{\circ} \mathrm{C}$ and stirred for 4 hours at $0{ }^{\circ} \mathrm{C}$. The reaction was allowed to warm to room temperature and quenched with water. The product was extracted with dichloromethane, and the combined organic layers were washed with brine, and then dried over anhydrous $\mathrm{MgSO}_{4}$. The slurry was filtered and the solvent was removed under vacuum. The residue was purified by silica gel column chromatography using a mixture of ethyl acetate and hexanes as eluent. Yellow oil (710 mg, 40\%). ${ }^{1} \mathrm{H}$ NMR (300 MHz, $\left.\mathrm{CDCl}_{3}\right): 7.32(\mathrm{~d}, \mathrm{~J}=7.5 \mathrm{~Hz}, 1 \mathrm{H}), 7.12$ (t, J = $7.2 \mathrm{~Hz}, 1 \mathrm{H}), 6.77(\mathrm{t}, \mathrm{J}=7.5 \mathrm{~Hz}, 1 \mathrm{H}), 6.71(\mathrm{~d}, \mathrm{~J}=7.8 \mathrm{~Hz}, 1 \mathrm{H}), 4.12(\mathrm{br} \mathrm{s}, 2 \mathrm{H}), 2.10(\mathrm{~d}, \mathrm{~J}=0.9$ $\mathrm{Hz}, 6 \mathrm{H})$.

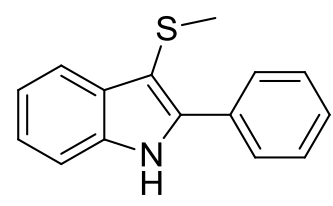

3-(Methylthio)-2-phenyl-1H-indole 8: Prepared using the previously prescribed indole synthesis. Brown solid (103 mg, 42\%). ${ }^{1} \mathrm{H}$ NMR (300 MHz, $\left.\mathrm{CDCl}_{3}\right): 8.30$ (br s, 1H), 7.81-7.85 $(\mathrm{m}, 3 \mathrm{H}), 7.48(\mathrm{t}, \mathrm{J}=6.0 \mathrm{~Hz}, 2 \mathrm{H}), 7.38-7.41(\mathrm{~m}, 2 \mathrm{H}), 7.21-7.30(\mathrm{~m}, 2 \mathrm{H}), 2.29(\mathrm{~s}, 3 \mathrm{H}) .{ }^{13} \mathrm{C}$ NMR 
(100 MHz, $\left.\mathrm{CDCl}_{3}\right):$ 139.7, 135.6, 131.9, 130.9, 128.6, 128.3, 128.1, 123.0, 120.6, 119.6, 111.1,

104.9, 19.72, 19.70. HRMS EI (m/z): calcd. for $\mathrm{C}_{15} \mathrm{H}_{13} \mathrm{NS}$ [ $\left.\mathrm{M}^{+}\right]$, 239.0769; found, 239.0772 .

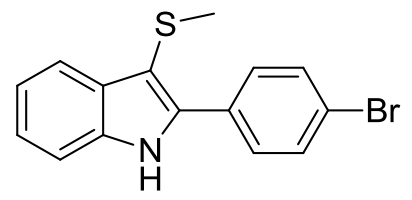

2-(4-Bromophenyl)-3-(methylthio)-1H-indole 9: Prepared using the previously prescribed indole synthesis. Brown solid (240 mg, 78\%). ${ }^{1} \mathrm{H}$ NMR $\left(300 \mathrm{MHz}, \mathrm{CDCl}_{3}\right): 8.31$ (br s, 1H), $7.80(\mathrm{dd}, \mathrm{J}=6.5,2.2 \mathrm{~Hz}, 1 \mathrm{H}), 7.71(\mathrm{dt}, \mathrm{J}=8.4,2.4 \mathrm{~Hz}, 2 \mathrm{H}), 7.59(\mathrm{dt}, \mathrm{J}=8.4,1.8 \mathrm{~Hz}$, 2H), $7.37(\mathrm{dd}, \mathrm{J}=6.9,2.4 \mathrm{~Hz}, 1 \mathrm{H}), 7.19-7.29(\mathrm{~m}, 2 \mathrm{H}), 2.28(\mathrm{~s}, 3 \mathrm{H}) .{ }^{13} \mathrm{C} \mathrm{NMR}(100 \mathrm{MHz}$, $\left.\mathrm{CDCl}_{3}\right): 138.4,135.6,131.8,130.82,130.76,129.5,123.3,122.4,120.8,119.7,111.1,105.5$, 19.7, 19.6. HRMS EI (m/z): calcd. for $\mathrm{C}_{15} \mathrm{H}_{12} \mathrm{BrNS}$ [ $\left.\mathrm{M}^{+}\right]$, 316.9874; found, 316.9878 .<smiles>O=Cc1ccccc1I</smiles>

2-Iodobenzaldehyde: To a solution of 2-iodobenzyl alcohol (2.0 g, $8.5 \mathrm{mmol})$ in dry dichloromethane $(40.0 \mathrm{~mL})$ was added $\geq 90 \%$ activated manganese(IV) oxide ( $7.5 \mathrm{~g}, 9$ equiv.). The reaction slurry was stirred overnight at room temperature then filtered through a sintered filter funnel and the pad was washed with dichloromethane. The filtrate was concentrated under vacuum, and the resulting solid was pure as indicated by TLC and ${ }^{1} \mathrm{H}$ NMR. Yellow solid (1.9 g, 93\%). ${ }^{1} \mathrm{H}$ NMR (300 MHz, $\mathrm{CDCl}_{3}$ ): 10.06 (s, 1H), 7.95 (d, J = 7.8 Hz, 1H), 7.88 (dd, J = 7.8, 1.7 $\mathrm{Hz}, 1 \mathrm{H}), 7.46(\mathrm{t}, \mathrm{J}=7.8,0.6 \mathrm{~Hz}, 1 \mathrm{H}), 7.28(\mathrm{td}, \mathrm{J}=7.8,1.8 \mathrm{~Hz}, 1 \mathrm{H})$.<smiles>COc1cc(I)c(C=O)cc1OC</smiles> 
2-Iodo-4,5-dimethoxybenzaldehyde: ${ }^{25}$ To a suspension of 3,4-dimethoxybenzaldehyde (358 mg, $2.15 \mathrm{mmol}$ ) and silver(I) triflouroacetate $(527 \mathrm{mg}, 2.39 \mathrm{mmol})$ in dry dichloromethane (15 mL) was slowly added a solution of iodine $(603 \mathrm{mg}, 2.38 \mathrm{mmol})$ in dry dichloromethane (35 $\mathrm{mL}$ ) via cannula over 4 hours. The reaction suspension was heated to reflux overnight, cooled and filtered through Celite ${ }^{\circledR}$, and the filtrate was washed with saturated sodium thiosulfate. The organic layer was dried over anhydrous $\mathrm{MgSO}_{4}$ and filtered. The filtrate was concentrated under vacuum, and the residue was purified by silica gel column chromatography using a mixture of ethyl acetate and hexanes as eluent. Yellow solid (480 mg, 76\%). ${ }^{1} \mathrm{H} \mathrm{NMR}\left(300 \mathrm{MHz}, \mathrm{CDCl}_{3}\right)$ : $9.86(\mathrm{~s}, 1 \mathrm{H}), 7.41(\mathrm{~s}, 1 \mathrm{H}), 7.30(\mathrm{~s}, 1 \mathrm{H}), 3.96(\mathrm{~s}, 3 \mathrm{H}), 3.92(\mathrm{~s}, 3 \mathrm{H})$.<smiles>COc1cccc(C=O)c1I</smiles>

2-Iodo-3-methoxybenzaldehyde: ${ }^{26}$ To a solution of $N, N, N^{\prime}$-trimethylethylenediamine $(0.42 \mathrm{~mL}, 3.3 \mathrm{mmol})$ in $\mathrm{THF}(8.0 \mathrm{~mL})$ at $-10^{\circ} \mathrm{C}$ was added a $2.5 \mathrm{M}$ solution of n-butyllithium in hexane $(1.3 \mathrm{~mL}, 3.3 \mathrm{mmol})$ dropwise. After 15 minutes of stirring at $-10{ }^{\circ} \mathrm{C}$, meta-anisaldehyde $(0.36 \mathrm{~mL}, 2.95 \mathrm{mmol})$ was added slowly to the reaction solution. After 15 minutes of stirring at $10{ }^{\circ} \mathrm{C}$, a $2.5 \mathrm{M}$ solution of n-butyllithium in hexane $(3.5 \mathrm{~mL}, 8.7 \mathrm{mmol})$ was added dropwise to the reaction solution. The orange reaction solution was stirred for $1 \frac{1 / 2}{2}$ hours at $-10^{\circ} \mathrm{C}$, then cooled to $-78^{\circ} \mathrm{C}$. To the reaction solution was added a solution of iodine $(2.34 \mathrm{~g}, 9.22 \mathrm{mmol})$ in THF (10 mL) slowly. The reaction was allowed to warm to room temperature and stirred for 2 hours. The reaction was quenched with $1 \mathrm{~N} \mathrm{HCl}$, and extracted three times with diethyl ether. The combined ether layers were washed with saturated sodium thiosulfate, dried over anhydrous $\mathrm{MgSO}_{4}$ and filtered. The solvent was removed under vacuum, and the residue was purified by 
silica gel column chromatography using a mixture of ethyl acetate and hexanes as eluent.

Yellow solid (303.1 mg, 39\%). ${ }^{1} \mathrm{H}$ NMR (300 MHz, $\left.\mathrm{CDCl}_{3}\right): 10.17$ (s, 1H), 7.47 (dd, J = 7.5, 1.2 Hz, 1H), 7.37 (t, J = 7.8 Hz, 1H), 7.04 (dd, J = 7.8, 0.9 Hz, 1H), 3.94 (s, 3H).

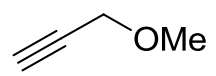

Methyl propargyl ether: ${ }^{28}$ To a mixture of propargyl alcohol $(20.0 \mathrm{~mL})$, and water $(15.0 \mathrm{~mL})$ in air was added a $50 \%$ solution of sodium hydroxide in water $(76 \mathrm{~mL})$ slowly while maintaining a temperature $<60^{\circ} \mathrm{C}$. To the slurry was added dimethylsulfate $(19.5 \mathrm{~mL})$ slowly while maintaining a temperature $<60{ }^{\circ} \mathrm{C}$. The reaction slurry was carefully heated to $50-55^{\circ} \mathrm{C}$ for 2 hours. The crude reaction slurry was distilled at $65{ }^{\circ} \mathrm{C}$. Colorless liquid $(10.8 \mathrm{~g}, 45 \%)$. ${ }^{1} \mathrm{H}$ $\operatorname{NMR}\left(400 \mathrm{MHz}, \mathrm{CDCl}_{3}\right): 4.10(\mathrm{~d}, \mathrm{~J}=2.4 \mathrm{~Hz}, 2 \mathrm{H}), 3.40(\mathrm{~s}, 3 \mathrm{H}), 2.47$ (t, J = 2.4 Hz, 1H).

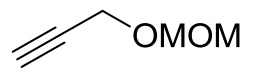

Methoxymethyl propargyl ether: ${ }^{29}$ To a solution of propargyl alcohol $(0.52 \mathrm{~mL}, 8.93$ mmol) and N,N-diisopropylethylamine $(4.7 \mathrm{~mL}, 27 \mathrm{mmol})$ in dichloromethane $(40.0 \mathrm{~mL})$ at -15 ${ }^{\circ} \mathrm{C}$ was added a solution of 2.1 $\mathrm{M}$ solution of $\mathrm{MOMCl}$ in toluene $(6.4 \mathrm{~mL}, 13.4 \mathrm{mmol})$ in dichloromethane $(10.0 \mathrm{~mL})$ slowly. The reaction was allowed to warm to room temperature and stirred overnight. The mixture was diluted with dichloromethane and washed with water, $1 \mathrm{~N}$ $\mathrm{HCl}$, saturated $\mathrm{NaHCO}_{3}$, water, and brine respectively. The organic layer was dried over anhydrous $\mathrm{MgSO}_{4}$, filtered, and concentrated under vacuum. Approximately a $20 \%$ solution in toluene $(1.1 \mathrm{~g}, \sim 25 \%) .{ }^{1} \mathrm{H}$ NMR $\left(400 \mathrm{MHz}, \mathrm{CDCl}_{3}\right): 4.71(\mathrm{~s}, 2 \mathrm{H}), 4.21(\mathrm{~d}, \mathrm{~J}=2.8 \mathrm{~Hz}, 2 \mathrm{H})$, $3.38(\mathrm{~s}, 3 \mathrm{H}), 2.41(\mathrm{t}, \mathrm{J}=2.8 \mathrm{~Hz}, 1 \mathrm{H})$. 


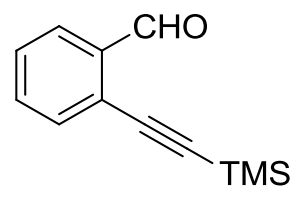

2-((Trimethylsilyl)ethynyl)benzaldehyde 10a: ${ }^{30}$ Prepared using the previously prescribed Sonogashira coupling conditions, at $50{ }^{\circ} \mathrm{C}$, with 2-bromobenzaldehyde. Brown solid (156 mg, 69\%). ${ }^{1} \mathrm{H}$ NMR (300 MHz, $\left.\mathrm{CDCl}_{3}\right): 10.56(\mathrm{~s}, 1 \mathrm{H}), 7.91(\mathrm{~d}, \mathrm{~J}=6.0 \mathrm{~Hz}, 1 \mathrm{H}), 7.51-7.59$ (m, 2H), $7.43(\mathrm{t}, \mathrm{J}=8.4 \mathrm{~Hz}, 1 \mathrm{H}), 0.27(\mathrm{~s}, 9 \mathrm{H})$.

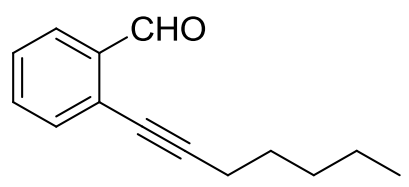

2-(Hept-1-yn-1-yl)benzaldehyde 10b: ${ }^{31}$ Prepared using the previously prescribed Sonogashira coupling conditions with 2-iodobenzaldehyde. Green oil (1.28 g, 96\%). ${ }^{1} \mathrm{H}$ NMR $\left(300 \mathrm{MHz}, \mathrm{CDCl}_{3}\right): 10.54(\mathrm{~d}, \mathrm{~J}=0.3 \mathrm{~Hz}, 1 \mathrm{H}), 7.88(\mathrm{~d}, \mathrm{~J}=7.8 \mathrm{~Hz}, 1 \mathrm{H}), 7.48-7.52(\mathrm{~m}, 2 \mathrm{H}), 7.34-$ $7.40(\mathrm{~m}, 1 \mathrm{H}), 2.47(\mathrm{t}, \mathrm{J}=7.2 \mathrm{~Hz}, 2 \mathrm{H}), 1.38-1.64(\mathrm{~m}, 6 \mathrm{H}), 0.93(\mathrm{t}, \mathrm{J}=7.2 \mathrm{~Hz}, 3 \mathrm{H})$.

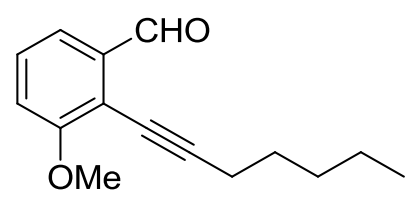

2-(Hept-1-yn-1-yl)-3-methoxybenzaldehyde 10c: ${ }^{31}$ Prepared using the previously prescribed Sonogashira coupling conditions, at $50{ }^{\circ} \mathrm{C}$, with 2-iodo-3-methoxybenzaldehyde. Yellow oil (140 mg, 53\%). ${ }^{1} \mathrm{H}$ NMR (300 MHz, $\left.\mathrm{CDCl}_{3}\right)$ : $10.55(\mathrm{~d}, \mathrm{~J}=0.9 \mathrm{~Hz}, 1 \mathrm{H}), 7.50$ (dd, J = 7.8, $0.9 \mathrm{~Hz}, 1 \mathrm{H}), 7.33(\mathrm{t}, \mathrm{J}=8.1 \mathrm{~Hz}, 1 \mathrm{H}), 7.09(\mathrm{~d}, \mathrm{~J}=8.1 \mathrm{~Hz}, 1 \mathrm{H}), 3.92(\mathrm{~s}, 3 \mathrm{H}), 2.55(\mathrm{t}, \mathrm{J}=7.2$ $\mathrm{Hz}, 2 \mathrm{H}), 1.36-1.70(\mathrm{~m}, 6 \mathrm{H}), 0.93(\mathrm{t}, \mathrm{J}=7.2 \mathrm{~Hz}, 3 \mathrm{H})$. 


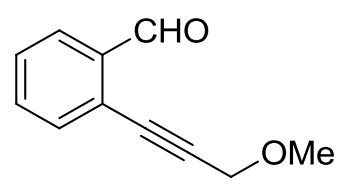

2-(3-Methoxyprop-1-yn-1-yl)benzaldehyde 10d: ${ }^{32}$ Prepared in THF using the previously prescribed Sonogashira coupling conditions with 2-bromobenzaldehyde. Yellow oil (141 mg, 52\%). ${ }^{1} \mathrm{H}$ NMR (300 MHz, $\left.\mathrm{CDCl}_{3}\right): 10.53(\mathrm{~s}, 1 \mathrm{H}), 7.92(\mathrm{~d}, \mathrm{~J}=7.5 \mathrm{~Hz}, 1 \mathrm{H}), 7.52-7.59$ (m, 2H), 7.41-7.48 (m, 1H), 4.39 (s, 2H), $3.48(\mathrm{~s}, 3 \mathrm{H})$.

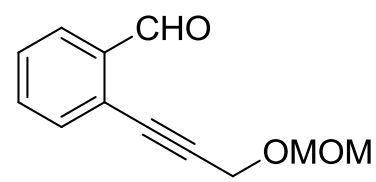

2-(3-(Methoxymethoxy)prop-1-yn-1-yl)benzaldehyde 10e: ${ }^{32}$ Prepared in THF using the previously prescribed Sonogashira coupling conditions with 2-bromobenzaldehyde. Product was not isolable from the alkynyl starting material. Brown oil $(85 \mathrm{mg}, \sim 40 \%) .{ }^{1} \mathrm{H}$ NMR $(300$ $\left.\mathrm{MHz}, \mathrm{CDCl}_{3}\right): 10.52(\mathrm{~s}, 1 \mathrm{H}), 7.92(\mathrm{~d}, \mathrm{~J}=7.5 \mathrm{~Hz}, 1 \mathrm{H}), 7.52-7.59(\mathrm{~m}, 2 \mathrm{H}), 7.42-7.48(\mathrm{~m}, 1 \mathrm{H})$, 4.79 (s, 2H), 4.52 (s, 2H), 3.43 (s, 3H). ${ }^{13} \mathrm{C}$ NMR (75 MHz, CDCl3): 191.6, 136.2, 133.8, 133.6, $129.0,127.3,126.2,95.2,95.0,92.1,55.8,54.9$.<smiles>CCOC(C#Cc1ccccc1C=O)OCC</smiles>

2-(3,3-Diethoxyprop-1-yn-1-yl)benzaldehyde $10 \mathbf{f :}^{32}$ Prepared in THF using the previously prescribed Sonogashira coupling conditions with 2-bromobenzaldehyde. Yellow oil (120 mg, 30\%). ${ }^{1} \mathrm{H}$ NMR (300 MHz, $\mathrm{CDCl}_{3}$ ): 10.52 (s, 1H), 7.93 (d, J = $\left.7.5 \mathrm{~Hz}, 1 \mathrm{H}\right)$, 7.54-7.63 (m, 2H), 7.45-7.50 (m, 1H), $5.55(\mathrm{~s}, 1 \mathrm{H}), 3.64-3.90(\mathrm{~m}, 4 \mathrm{H}), 1.29(\mathrm{~s}, 6 \mathrm{H})$. 


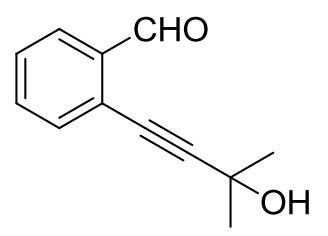

2-(3-Hydroxy-3-methylbut-1-yn-1-yl)benzaldehyde 10g: ${ }^{31}$ Prepared using the previously prescribed Sonogashira coupling conditions with 2-iodobenzaldehyde. Orange oil (214 mg, 88\%). ${ }^{1} \mathrm{H}$ NMR (300 MHz, $\left.\mathrm{CDCl}_{3}\right): 10.48(\mathrm{~s}, 1 \mathrm{H}), 7.88$ (d, J = 8.1 Hz, 1H), 7.49-7.53 (m, 2H), 7.38-7.43 (m, 1H), 3.10 (br s, 1H), $1.66(\mathrm{~s}, 6 \mathrm{H})$.

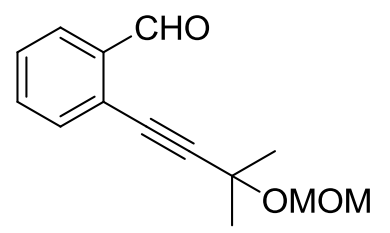

2-(3-(Methoxymethoxy)-3-methylbut-1-yn-1-yl)benzaldehyde 10g': ${ }^{33}$ To a solution of 2.1 M MOMCl in toluene $(1.1 \mathrm{~mL}, 2.3 \mathrm{mmol})$ was added $\mathbf{1 0 g}(207 \mathrm{mg}, 1.1 \mathrm{mmol})$ followed by $\mathrm{N}, \mathrm{N}$-diisopropylethylamine $(0.24 \mathrm{~mL}, 1.4 \mathrm{mmol})$. The reaction was stirred overnight, then partitioned between ethyl acetate and saturated $\mathrm{NH}_{4} \mathrm{Cl}$ and stirred for 10 minutes. The organic layer was removed and washed with saturated $\mathrm{NaHCO}_{3}$, brine, and dried over anhydrous $\mathrm{MgSO}_{4}$. The slurry was filtered and concentrated under vacuum. Orange oil $(231 \mathrm{mg}, 91 \%) .{ }^{1} \mathrm{H}$ NMR $\left(300 \mathrm{MHz}, \mathrm{CDCl}_{3}\right): 10.51(\mathrm{~d}, \mathrm{~J}=0.6 \mathrm{~Hz}, 1 \mathrm{H}), 7.92(\mathrm{dt}, \mathrm{J}=7.8,0.9 \mathrm{~Hz}, 1 \mathrm{H}), 7.53-7.56(\mathrm{~m}, 2 \mathrm{H})$, 7.41-7.47 (m, 1H), $4.97(\mathrm{~s}, 2 \mathrm{H}), 3.43(\mathrm{~s}, 3 \mathrm{H}), 1.65(\mathrm{~s}, 6 \mathrm{H})$.<smiles>COC(C)C#Cc1ccccc1C=O</smiles> 
2-(3-(Methoxymethoxy)but-1-yn-1-yl)benzaldehyde 10h: ${ }^{31}$ The unprotected alcohol, 2-(3-hydroxybut-1-yn-1-yl)benzaldehyde, was prepared using the previously prescribed Sonogashira coupling conditions with 2-iodobenzaldehyde and 3-butyn-2-ol, but proved to be unstable and was quickly MOM protected as follows: ${ }^{33}$ To a solution of $2.1 \mathrm{M} \mathrm{MOMCl}$ in toluene (0.95 mL, $2.0 \mathrm{mmol})$ was added 2-(3-hydroxybut-1-yn-1-yl)benzaldehyde ( $1.0 \mathrm{mmol})$ followed by $\mathrm{N}, \mathrm{N}$-diisopropylethylamine $(0.23 \mathrm{~mL}, 1.3 \mathrm{mmol})$. The reaction was stirred overnight, then partitioned between ethyl acetate and saturated $\mathrm{NH}_{4} \mathrm{Cl}$ and stirred for 10 minutes. The organic layer was removed and washed with saturated $\mathrm{NaHCO}_{3}$, brine, and dried over anhydrous $\mathrm{MgSO}_{4}$. The slurry was filtered and concentrated under vacuum. Orange/yellow oil (79 mg, 35\%). ${ }^{1} \mathrm{H}$ NMR (300 MHz, $\left.\mathrm{CDCl}_{3}\right): 10.52(\mathrm{~s}, 1 \mathrm{H}), 7.92(\mathrm{~d}, \mathrm{~J}=7.8 \mathrm{~Hz}, 1 \mathrm{H}), 7.52-7.57$ (m, 2H), 7.41-7.48 (m, 1H), 4.99, $4.68(\mathrm{ABq}, \mathrm{J}=6.6 \mathrm{~Hz}, 2 \mathrm{H}), 4.74(\mathrm{q}, \mathrm{J}=6.6 \mathrm{~Hz}, 1 \mathrm{H}), 3.43(\mathrm{~s}$, $3 \mathrm{H}), 1.59(\mathrm{~d}, \mathrm{~J}=6.6 \mathrm{~Hz}, 3 \mathrm{H})$.

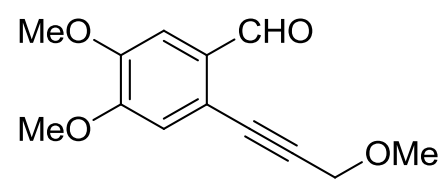

4,5-Dimethoxy-2-(3-methoxyprop-1-yn-1-yl)benzaldehyde 10i: ${ }^{31}$ Prepared in THF using the previously prescribed Sonogashira coupling conditions with 2-iodo-4,5dimethoxybenzaldehyde. Yellow solid (132 mg, 87\%). ${ }^{1} \mathrm{H}$ NMR (300 MHz, $\left.\mathrm{CDCl}_{3}\right): 10.38$ (s, 1H), 7.39 (s, 1H), $6.99(\mathrm{~s}, 1 \mathrm{H}), 4.38(\mathrm{~s}, 2 \mathrm{H}), 3.96(\mathrm{~s}, 3 \mathrm{H}), 3.95(\mathrm{~s}, 3 \mathrm{H}), 3.48(\mathrm{~s}, 3 \mathrm{H})$.

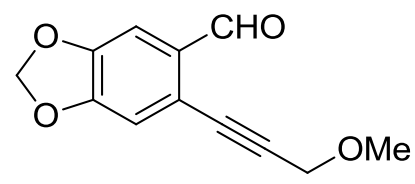


6-(3-Methoxyprop-1-yn-1-yl)benzo[d][1,3]dioxole-5-carbaldehyde $10 \mathrm{j}:{ }^{34}$ Prepared in THF at reflux using the previously prescribed Sonogashira coupling conditions with 6-bromo1,3-benzodioxole-5-carboxaldehyde. Tan solid (99 mg, 39\%). ${ }^{1} \mathrm{H}$ NMR (300 MHz, $\mathrm{CDCl}_{3}$ ): $10.36(\mathrm{~s}, 1 \mathrm{H}), 7.32(\mathrm{~s}, 1 \mathrm{H}), 6.94(\mathrm{~s}, 1 \mathrm{H}), 6.08(\mathrm{~s}, 2 \mathrm{H}), 4.36(\mathrm{~s}, 2 \mathrm{H}), 3.46(\mathrm{~s}, 3 \mathrm{H})$.<smiles>COCC#Cc1c(C=O)cccc1OC</smiles>

3-Methoxy-2-(3-methoxyprop-1-yn-1-yl)benzaldehyde 10k: ${ }^{31}$ Prepared using the previously prescribed Sonogashira coupling conditions, at $50{ }^{\circ} \mathrm{C}$, with 2 -iodo-3methoxybenzaldehyde. Orange solid (38 mg, 29\%). ${ }^{1} \mathrm{H} \mathrm{NMR}\left(300 \mathrm{MHz}, \mathrm{CDCl}_{3}\right): 10.54$ (s, $\left.1 \mathrm{H}\right)$, $7.53(\mathrm{dd}, \mathrm{J}=7.8,0.9 \mathrm{~Hz}, 1 \mathrm{H}), 7.40(\mathrm{t}, \mathrm{J}=7.8 \mathrm{~Hz}, 1 \mathrm{H}), 7.12(\mathrm{~d}, \mathrm{~J}=8.4 \mathrm{~Hz}, 1 \mathrm{H}), 4.45$ (s, 2H), $3.94(\mathrm{~s}, 3 \mathrm{H}), 3.49(\mathrm{~s}, 3 \mathrm{H})$.

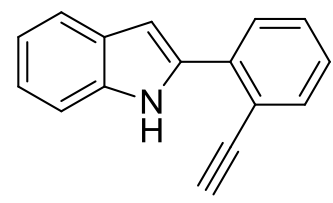

2-(2-Ethynylphenyl)-1H-indole 11a: Prepared using the previously prescribed indole synthesis. Brown solid (38 mg, 18\%). ${ }^{1} \mathrm{H}$ NMR (300 MHz, $\left.\mathrm{CDCl}_{3}\right): 9.52$ (br s, 1H), $7.75(\mathrm{~d}, \mathrm{~J}=$ 8.1 Hz, 1H), 7.59-7.66 (m, 2H), 7.39-7.43 (m, 2H), 7.19-7.28 (m, 2H), 7.12 (t, J = 7.8 Hz, 1H), $6.96(\mathrm{~d}, \mathrm{~J}=1.5 \mathrm{~Hz}, 1 \mathrm{H}), 3.44(\mathrm{~s}, 1 \mathrm{H})$.

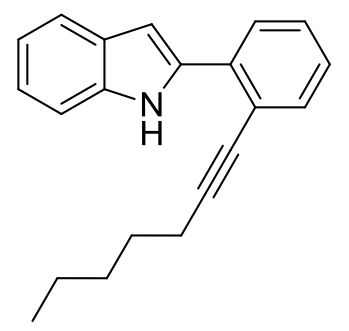


2-(2-(Hept-1-yn-1-yl)phenyl)-1H-indole 11b: Prepared using the previously prescribed indole synthesis. Yellow solid (68 mg, 45\%). ${ }^{1} \mathrm{H}$ NMR (300 MHz, $\mathrm{CDCl}_{3}$ ): 9.80 (br s, 1H), 7.76 $(\mathrm{d}, \mathrm{J}=8.1 \mathrm{~Hz}, 1 \mathrm{H}), 7.64(\mathrm{~d}, \mathrm{~J}=7.8 \mathrm{~Hz}, 1 \mathrm{H}), 7.49(\mathrm{~d}, \mathrm{~J}=7.5 \mathrm{~Hz}, 1 \mathrm{H}), 7.29-7.37(\mathrm{~m}, 2 \mathrm{H}), 7.17-$ $7.23(\mathrm{~m}, 2 \mathrm{H}), 7.11(\mathrm{t}, \mathrm{J}=7.5 \mathrm{~Hz}, 1 \mathrm{H}), 6.93(\mathrm{~d}, \mathrm{~J}=1.8 \mathrm{~Hz}, 1 \mathrm{H}), 2.52(\mathrm{t}, \mathrm{J}=7.2 \mathrm{~Hz}, 2 \mathrm{H}), 1.35-$ $1.73(\mathrm{~m}, 6 \mathrm{H}), 0.90(\mathrm{t}, \mathrm{J}=7.2 \mathrm{~Hz}, 3 \mathrm{H})$.

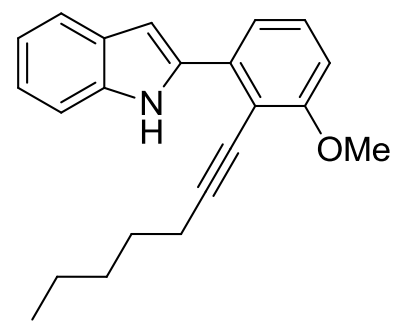

2-(2-(Hept-1-yn-1-yl)-3-methoxyphenyl)-1H-indole 11c: Prepared using the previously prescribed indole synthesis. Yellow solid (80 mg, 57\%). ${ }^{1} \mathrm{H}$ NMR (300 MHz, $\mathrm{CDCl}_{3}$ ): 9.85 (br s, 1H), $7.64(\mathrm{~d}, \mathrm{~J}=7.8 \mathrm{~Hz}, 1 \mathrm{H}), 7.35-7.39(\mathrm{~m}, 2 \mathrm{H}), 7.27(\mathrm{t}, \mathrm{J}=8.1 \mathrm{~Hz}, 1 \mathrm{H}), 7.19(\mathrm{td}, \mathrm{J}=8.1,1.2$ $\mathrm{Hz}, 1 \mathrm{H}), 7.11(\mathrm{td}, \mathrm{J}=7.8,0.9 \mathrm{~Hz}, 1 \mathrm{H}), 6.93(\mathrm{dd}, \mathrm{J}=2.1,0.9 \mathrm{~Hz}, 1 \mathrm{H}), 6.80(\mathrm{~d}, \mathrm{~J}=8.1 \mathrm{~Hz}, 1 \mathrm{H})$, $3.91(\mathrm{~s}, 3 \mathrm{H}), 2.57(\mathrm{t}, \mathrm{J}=7.2 \mathrm{~Hz}, 2 \mathrm{H}), 1.34-1.75(\mathrm{~m}, 6 \mathrm{H}), 0.89(\mathrm{t}, \mathrm{J}=7.2 \mathrm{~Hz}, 3 \mathrm{H})$.

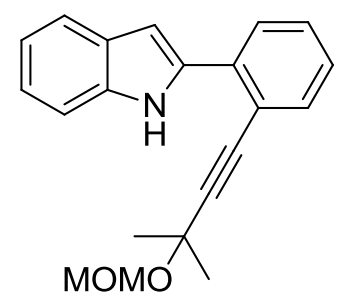

2-(2-(3-(Methoxymethoxy)-3-methylbut-1-yn-1-yl)phenyl)-1H-indole 11d: Prepared using the previously prescribed indole synthesis. Yellow oil (89 mg, 53\%). ${ }^{1} \mathrm{H}$ NMR (300 MHz, $\left.\mathrm{CDCl}_{3}\right): 10.07$ (br s, $\left.1 \mathrm{H}\right), 7.78(\mathrm{~d}, \mathrm{~J}=8.1 \mathrm{~Hz}, 1 \mathrm{H}), 7.64(\mathrm{~d}, \mathrm{~J}=7.8 \mathrm{~Hz}, 1 \mathrm{H}), 7.52(\mathrm{dd}, \mathrm{J}=7.5,1.2$ $\mathrm{Hz}, 1 \mathrm{H}), 7.43(\mathrm{~d}, \mathrm{~J}=8.1 \mathrm{~Hz}, 1 \mathrm{H}), 7.37(\mathrm{td}, \mathrm{J}=7.5,1.5 \mathrm{~Hz}, 1 \mathrm{H}), 7.16-7.26(\mathrm{~m}, 2 \mathrm{H}), 7.11(\mathrm{t}, \mathrm{J}=$ $7.8 \mathrm{~Hz}, 1 \mathrm{H}), 6.89(\mathrm{~d}, \mathrm{~J}=2.1 \mathrm{~Hz}, 1 \mathrm{H}), 5.03(\mathrm{~s}, 2 \mathrm{H}), 3.49(\mathrm{~s}, 3 \mathrm{H}), 1.64(\mathrm{~s}, 6 \mathrm{H})$. 


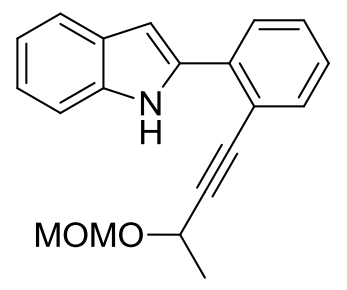

2-(2-(3-(Methoxymethoxy)but-1-yn-1-yl)phenyl)-1H-indole 11e: Prepared using the previously prescribed indole synthesis. Red oil (71 mg, 64\%). ${ }^{1} \mathrm{H}$ NMR (300 MHz, $\left.\mathrm{CDCl}_{3}\right)$ : 9.91 (br s, 1H), $7.78(\mathrm{~d}, \mathrm{~J}=8.1 \mathrm{~Hz}, 1 \mathrm{H}), 7.64(\mathrm{~d}, \mathrm{~J}=7.8 \mathrm{~Hz}, 1 \mathrm{H}), 7.52$ (d, J = 7.8 Hz, 1H), 7.35$7.44(\mathrm{~m}, 2 \mathrm{H}), 7.17-7.26(\mathrm{~m}, 2 \mathrm{H}), 7.11(\mathrm{t}, \mathrm{J}=7.8 \mathrm{~Hz}, 1 \mathrm{H}), 6.92(\mathrm{~d}, \mathrm{~J}=1.5 \mathrm{~Hz}, 1 \mathrm{H}), 5.00,4.79$ $(\mathrm{ABq}, \mathrm{J}=6.9 \mathrm{~Hz}, 2 \mathrm{H}), 4.69(\mathrm{q}, \mathrm{J}=6.6,1 \mathrm{H}), 3.46(\mathrm{~s}, 3 \mathrm{H}), 1.61(\mathrm{~d}, \mathrm{~J}=6.6 \mathrm{~Hz}, 3 \mathrm{H})$.

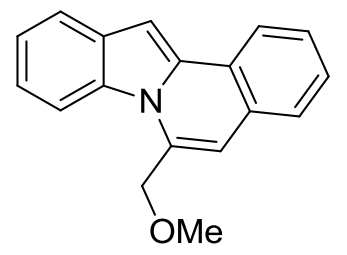

6-(Methoxymethyl)indolo[2,1-a]isoquinoline 12a: Prepared using the previously prescribed indole synthesis. Brown solid (66 mg, 31\%). ${ }^{1} \mathrm{H} \mathrm{NMR}\left(300 \mathrm{MHz}, \mathrm{CDCl}_{3}\right): 8.14$ (d, J $=7.8 \mathrm{~Hz}, 1 \mathrm{H}), 8.06(\mathrm{dd}, \mathrm{J}=7.2,2.1 \mathrm{~Hz}, 1 \mathrm{H}), 7.81(\mathrm{dd}, \mathrm{J}=6.6,2.1 \mathrm{~Hz}, 1 \mathrm{H}), 7.41-7.54(\mathrm{~m}, 3 \mathrm{H})$, 7.30-7.35 (m, 3H), 6.67 (s, 1H), 4.94 (s, 2H), 3.51 (s, 3H). ${ }^{13} \mathrm{C} \mathrm{NMR}\left(75 \mathrm{MHz}, \mathrm{CDCl}_{3}\right)$ : 136.7, 134.6, 132.5, 130.0, 128.7, 128.0, 127.0, 126.4, 123.8, 122.4, 121.7, 120.9, 115.3, 112.0, 95.3, 72.8, 58.1. HRMS ACPI (m/z): calcd for $\mathrm{C}_{18} \mathrm{H}_{16} \mathrm{NO}\left[\mathrm{M}^{+}+\mathrm{H}\right], 262.1226$; found, 262.1228 .<smiles>COCc1cc2ccccc2c2cc3ccccc3n12</smiles> 
6-((Methoxymethoxy)methyl)indolo[2,1-a]isoquinoline 12b: Prepared using the previously prescribed indole synthesis. Brown solid (137 mg, 29\%). ${ }^{1} \mathrm{H}$ NMR (300 MHz, $\left.\mathrm{CDCl}_{3}\right):$ 8.17-8.22 (m, 2H), $7.84(\mathrm{dd}, \mathrm{J}=6.3,2.7 \mathrm{~Hz}, 1 \mathrm{H}), 7.47-7.56(\mathrm{~m}, 3 \mathrm{H}), 7.35-7.38(\mathrm{~m}$, 3H), $6.77(\mathrm{~s}, 1 \mathrm{H}), 5.17(\mathrm{~s}, 2 \mathrm{H}), 4.86(\mathrm{~s}, 2 \mathrm{H}), 3.50(\mathrm{~s}, 3 \mathrm{H}) .{ }^{13} \mathrm{C} \mathrm{NMR}\left(75 \mathrm{MHz}, \mathrm{CDCl}_{3}\right): 136.2$, $133.9,131.9,129.4,128.2,127.5,126.5,125.9,123.3,121.9,121.1,120.4,114.7,111.5,105.4$, 94.72, 94.70, 66.2, 55.8. HRMS ACPI (m/z): calcd for $\mathrm{C}_{19} \mathrm{H}_{18} \mathrm{NO}_{2}\left[\mathrm{M}^{+}+\mathrm{H}\right]$, 292.1332; found, 292.1335.

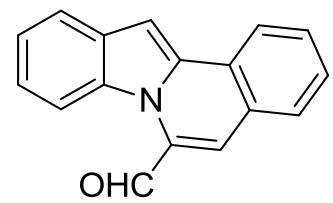

Indolo[2,1-a]isoquinoline-6-carbaldehyde 12c: Prepared using the previously prescribed indole synthesis. Yellow solid (127 mg, 22\%). ${ }^{1} \mathrm{H}$ NMR (300 $\left.\mathrm{MHz}, \mathrm{CDCl}_{3}\right): 10.59$ $(\mathrm{d}, \mathrm{J}=8.1 \mathrm{~Hz}, 1 \mathrm{H}), 8.04(\mathrm{~d}, \mathrm{~J}=8.1 \mathrm{~Hz}, 1 \mathrm{H}), 7.47-7.60(\mathrm{~m}, 4 \mathrm{H}), 7.14-7.36(\mathrm{~m}, 3 \mathrm{H}), 6.66(\mathrm{~s}, 1 \mathrm{H})$, $6.58(\mathrm{~d}, \mathrm{~J}=7.8 \mathrm{~Hz}, 1 \mathrm{H}) .{ }^{13} \mathrm{C} \mathrm{NMR}\left(75 \mathrm{MHz}, \mathrm{CDCl}_{3}\right): 187.9,149.3,141.4,136.1,135.3,134.0$, 132.9, 131.7, 128.5, 127.0, 125.4, 123.3, 123.0, 121.4, 112.5, 109.2, 105.4, 100.8. HRMS ESI (m/z): calcd for $\mathrm{C}_{17} \mathrm{H}_{12} \mathrm{NO}\left[\mathrm{M}^{+}+\mathrm{H}\right], 246.0913$; found, 246.0915 .

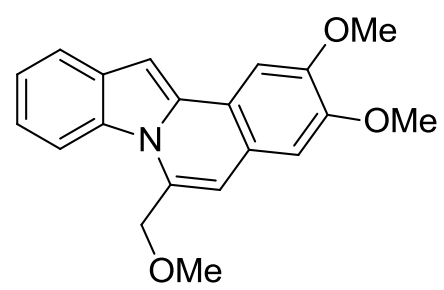

2,3-Dimethoxy-6-(methoxymethyl)indolo[2,1-a]isoquinoline 12d: Prepared using the previously prescribed indole synthesis. Yellow solid (117 mg, 65\%). ${ }^{1} \mathrm{H}$ NMR (300 MHz, $\left.\mathrm{CDCl}_{3}\right): 8.08(\mathrm{~d}, \mathrm{~J}=8.4 \mathrm{~Hz}, 1 \mathrm{H}), 7.80(\mathrm{~d}, \mathrm{~J}=6.6 \mathrm{~Hz}, 1 \mathrm{H}), 7.52(\mathrm{~s}, 1 \mathrm{H}), 7.24-7.35(\mathrm{~m}, 2 \mathrm{H}), 7.15$ 
(s, 1H), $6.96(\mathrm{~s}, 1 \mathrm{H}), 6.63(\mathrm{~s}, 1 \mathrm{H}), 4.95(\mathrm{~s}, 2 \mathrm{H}), 4.04(\mathrm{~s}, 3 \mathrm{H}), 3.97(\mathrm{~s}, 3 \mathrm{H}), 3.51(\mathrm{~s}, 3 \mathrm{H}) .{ }^{13} \mathrm{C}$ NMR (75 MHz, $\left.\mathrm{CDCl}_{3}\right):$ 149.7, 149.6, 136.3, 132.8, 132.7, 131.8, 129.7, 128.7, 128.6, 122.4, 121.9, 120.6, 120.1, 119.8, 114.9, 111.2, 107.6, 104.7, 92.7, 72.3, 57.6, 56.2, 56.1. HRMS ESI (m/z): calcd for $\mathrm{C}_{20} \mathrm{H}_{20} \mathrm{NO}_{3}\left[\mathrm{M}^{+}+\mathrm{H}\right], 322.1438$; found, 322.1437 .

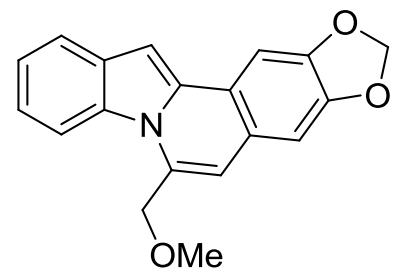

6-(Methoxymethyl)-[1,3]dioxolo[4,5-g]indolo[2,1-a]isoquinoline 12e: Prepared using the previously prescribed indole synthesis. Yellow solid (49 mg, 36\%). ${ }^{1} \mathrm{H}$ NMR (300 MHz, $\left.\mathrm{CDCl}_{3}\right): 8.08(\mathrm{~d}, \mathrm{~J}=8.4 \mathrm{~Hz}, 1 \mathrm{H}), 7.80(\mathrm{~d}, \mathrm{~J}=7.5 \mathrm{~Hz}, 1 \mathrm{H}), 7.56(\mathrm{~s}, 1 \mathrm{H}), 7.26-7.36(\mathrm{~m}, 2 \mathrm{H}), 7.13$ (s, 1H), $6.96(\mathrm{~s}, 1 \mathrm{H}), 6.62(\mathrm{~s}, 1 \mathrm{H}), 6.06(\mathrm{~s}, 2 \mathrm{H}), 4.97(\mathrm{~s}, 2 \mathrm{H}), 3.53(\mathrm{~s}, 3 \mathrm{H}) .{ }^{13} \mathrm{C} \mathrm{NMR}(75 \mathrm{MHz}$, $\left.\mathrm{CDCl}_{3}\right): 148.12,148.06,136.5,132.7,131.7,129.6,123.8,122.0,121.2,120.7,120.2,114.9$, 111.5, 105.1, 102.5, 101.5, 93.1, 72.3, 57.7. HRMS ESI (m/z): calcd for $\mathrm{C}_{19} \mathrm{H}_{16} \mathrm{NO}_{3}\left[\mathrm{M}^{+}+\mathrm{H}\right]$, 306.1125; found, 306.1127.

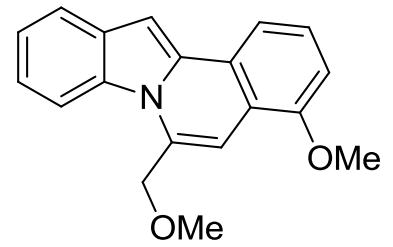

4-Methoxy-6-(methoxymethyl)indolo[2,1-a]isoquinoline 12f: Prepared using the previously prescribed indole synthesis. Tan solid (15 mg, 28\%). ${ }^{1} \mathrm{H} \mathrm{NMR}\left(300 \mathrm{MHz}, \mathrm{CDCl}_{3}\right)$ : $8.15(\mathrm{~d}, \mathrm{~J}=8.7 \mathrm{~Hz}, 1 \mathrm{H}), 7.76-7.84(\mathrm{~m}, 2 \mathrm{H}), 7.43(\mathrm{t}, \mathrm{J}=8.1 \mathrm{~Hz}, 1 \mathrm{H}), 7.31-7.35(\mathrm{~m}, 3 \mathrm{H}), 7.13(\mathrm{~s}$, 1H), $6.90(\mathrm{~d}, \mathrm{~J}=8.1 \mathrm{~Hz}, 1 \mathrm{H}), 5.01(\mathrm{~s}, 2 \mathrm{H}), 3.98(\mathrm{~s}, 3 \mathrm{H}), 3.52(\mathrm{~s}, 3 \mathrm{H}) .{ }^{13} \mathrm{C} \mathrm{NMR}(75 \mathrm{MHz}$, $\left.\mathrm{CDCl}_{3}\right):$ 155.0, 136.0, 133.2, 131.9, 129.4, 128.0, 127.0, 121.8, 121.1, 120.3, 118.5, 115.6, 
114.9, 107.4, 106.0, 105.4, 94.8, 72.5, 57.3, 55.6. HRMS ESI (m/z): calcd for $\mathrm{C}_{19} \mathrm{H}_{18} \mathrm{NO}_{2}\left[\mathrm{M}^{+}+\right.$ H], 292.1332; found, 292.1332 .

\section{References}

1. Gribble, G. W. Comprehensive Heterocyclic Chemistry II, Vol. 2.; Katritzky, A. R.; Rees, C. W.; Scriven, E. F. V., Eds.; Pergamon Press: Oxford, 1996, 207.

2. (a) Sundberg, R. J. Indoles; Academic Press: New York, 1996. (b) Gribble, G. W. J. Chem. Soc., Perkin Trans. I 2000, 1045-1075.

3. Taber, D. F.; Tirunahari, P. K. Tetrahedron, 2011, 67, 7195-7210.

4. Robinson, B. Chem. Rev. 1969, 69, 227.

5. Bartoli, G.; Palmieri, G. Tetrahedron Lett. 1989, 30, 2129-2132.

6. Gassman, P. G.; Van Bergen, T. J. J. Am. Chem. Soc. 1973, 95, 590.

7. Philips, R. R. Org. React. 1959, 10, 143.

8. Larock, R. C.; Yum, E. K.; Refvik, M. D. J. Org. Chem. 1998, 63, 7652-7662.

9. (a) Bressy, C.; Alberico, D.; Lautens, M. J. Am. Chem. Soc. 2005, 127, 13148. (b) Grimster, N. P.; Gauntlett, C.; Godfrey, C. R. A.; Gaunt, M. J. Angew. Chem. Int. Ed. 2005, 44, 3125. (c) Deprez, N. R.; Kalyani, D.; Krause, A.; Sanford, M. S. J. Am. Chem. Soc. 2006, 128, 4972.

10. Houlihan, W. J.; Uike, Y.; Parrino, V. A. J. Org. Chem. 1981, 46, 4511-4515.

11. Sridharan, V.; Parumal, S.; Avendaño, C.; Menéndez, J. C. Synlett 2006, 91-95.

12. (a) Kraus, G. A.; Guo, H. J. Org. Chem. 2009, 74, 5337-5341. (b) Kraus, G. A.; Guo, H. Org. Lett. 2008, 10(14), 3061-3063.

13. Goldbrunner, M.; Loidl, G.; Polossek, T.; Mannschreck, A.; von Angerer, E. J. Med. Chem. 1997, 40, 3524-3533.

14. Ogawa, M.; Morita, T.; Iibuchi, N.; Suzuki, H.; Tsutsui, H.; Kano, A.; Kato, S. PCT Int. Appl. 1999, WO 9954325 A1 19991028.

15. Ahmed, E.; Briseno, A. L.; Xia, Y.; Jenekhe, S. A. J. Am. Chem. Soc. 2008, 130, 11181119.

16. Elliott, I. W. Dibenzopyrrocoline Alkaloids Alkaloids (Academic Press) 1987, 31, 101116. 
17. (a) Bressy, C.; Alberico, D.; Lautens, M. J. Am. Chem. Soc. 2005, 127, 13148-13149. (b) Loetter, A. N. C.; Pathak, R.; Sello, T. S.; Fernandes, M. A.; van Otterlo, W. A. L.; de Koning, C. B. Tetrahedron 2007, 63, 2263-2274. (c) Sun, L. L.; Liao, Z. Y., Tang, R. Y.; Deng, C. L.; Zhang, X. G. J. Org. Chem. 2012, 77, 2850-2856.

18. Harada, R.; Nishida, N.; Uchiito, S.; Onozaki, Y.; Kurono, N.; Senboku, H.; Masao, T.; Ohkuma, T.; Orito, K. Eur. J. Org. Chem. 2012, 366-379.

19. (a) Verna, A. K.; Kesharwani, T.; Singh, J.; Tandon, V.; Larock, R. C. Angew. Chem. Int. Ed. 2009, 48, 1138-1143. (b) Verma, A. K.; Jha, R. R.; Chaudhary, R.; Tiwari, R. K.; Reddy, K. S. K.; Danodia, A. J. Org. Chem. 2012, 77, 8191-8205.

20. (a) Morimoto, K.; Hirano, K.; Satoh, T.; Miura, M. Org. Lett. 2010, 12, 2068-2071. (b) Li, Y.; Zhu, J.; Xie, H.; Li, S.; Peng, D.; Li, Z.; Wu, Y.; Gong, Y. Chem. Commun. 2012, 48, 3136-3138.

21. Mamane, V.; Hannen, P.; Fürstner, A. Chem.-A Eur. J. 2004, 10, 4556-4575.

22. Kraus, G. A.; Guo, H.; Kumar, G.; Pollock III, G.; Carruthers, H.; Chaudhary, D.; Beasley, J. Synthesis, 2010, 8, 1386-1393.

23. Hanessian, S.; Tyler, P. C.; Demailly, G.; Chapleur, Y. J. Am. Chem. Soc. 1981, 103, 6243-6246.

24. Gassman, P. G.; Gruetzmacher, G. J. Am. Chem. Soc. 1973, 95, 588-589.

25. Ridley, C. P.; Reddy, M. V. R.; Rocha, G.; Bushman, F. D.; Faulkner, D. J. Bioorg. Med. Chem. 2002, 10, 3285-3290.

26. Comins, D. L. and Brown, J. D. J. Org. Chem. 1984, 49, 1078-1083.

27. Claus, P. K.; Jaeger, E.; Setzer, A. Monatsh. Chem. 1985, 116, 1017-1026.

28. Perez, M.; Canoa, P.; Gómez, G.; Teijeira, M.; Fall, Y. Synthesis 2005, 3, 411-414.

29. Manes, A.; Stecko, S.; Mikołajczyk, P.; Soluch, M.; Furman, B.; Chmielewski, M. J. Org. Chem. 2010, 75, 7580-7587.

30. Park, J. H.; Bhilare, S. V.; Youn, S. W. Org. Lett. 2011, 13, 2228-2231.

31. Shu, X.-Z.; Zhao, S.-C.; Ji, K.-G.; Zheng, Z.-J.; Liu, X.-Y.; Liang, Y.-M. Eur. J. Org. Chem. 2009, 117-122.

32. Patti, R. K.; Duan, S.; Wang, Z.; Herndon, J. W. Tetrahedron Lett. 2011, 52, 4182-4185.

33. Berliner, M. A.; Belecki, K. J. Org. Chem. 2005, 70, 9618-9621.

34. Zhou, F.; Han, X.; Lu, X. J. Org. Chem. 2011, 76, 1491-1494. 
Chapter 2. BIO-BASED APPROACHES TO MALEIC ACID AND IONIC LIQUIDS

\section{Introduction}

The need for bio-based syntheses of commodity chemicals looms large as traditional fossil fuel supplies increasingly diminish. Further increases in energy demand exacerbate petroleum sources and highlight the growing concern for sustainability. Renewable feedstocks offer a sustainable solution to dwindling petroleum reserves and have been shown in the past decade to lead to possible replacements toward specialty chemicals. ${ }^{1}$ Recent advances in biorenewable chemistry demonstrate platform chemicals such as 5-hydroxymethylfurfural (HMF) to be likely solutions (Scheme 1). ${ }^{2}$ Our interest in biorenewables stemmed from a desire to build upon platform chemicals toward commodity and specialty chemicals. This approach was partitioned into two areas: 5-carbon sugars and 6-carbon sugars.

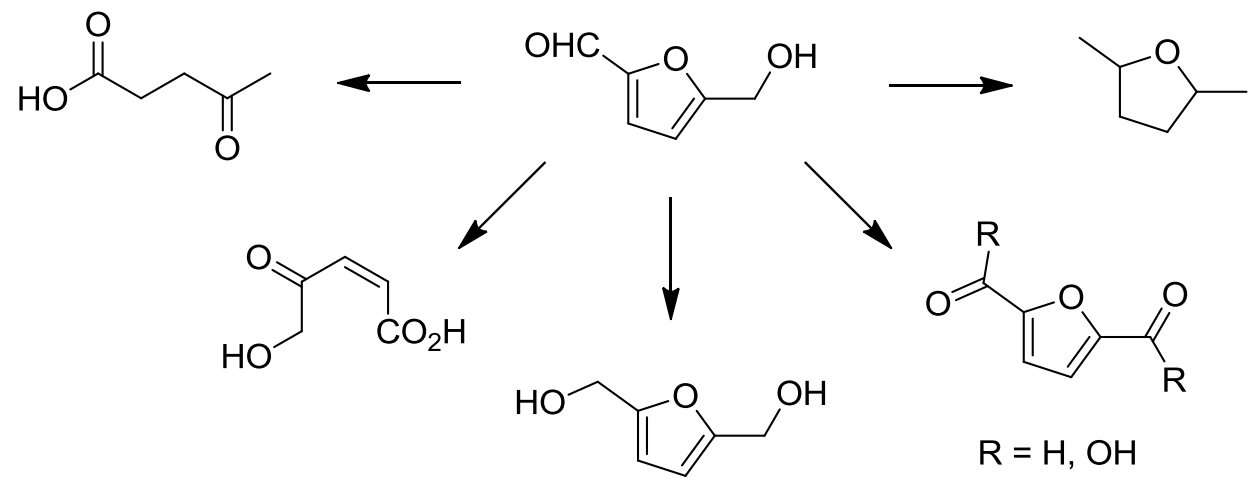

Scheme 1. Synthetic utility of HMF

Our initial interest in pentoses developed from a desire to synthesize commodity maleic acid and/or anhydride by bio-based means (Scheme 2). Maleic acid (1) and its anhydride are interconvertable: maleic acid is easily dehydrated to its anhydride through heating, while the anhydride can be hydrolyzed to the acid. Maleic acid is used in the production of fumaric acid through isomerization, while maleic anhydride is used as a copolymer in many polymerization 
processes. ${ }^{3}$ Both can also serve as dienophiles in Diels-Alder reactions as well as many other industrial processes. ${ }^{4}$ Therefore, a synthesis of one can readily lead to the other. Currently, maleic acid is industrially produced from the more commercially useful maleic anhydride which is synthesized by catalytic oxidation of hydrocarbons, of which benzene is the major precursor. ${ }^{3}$ We sought to start from bio-based platform chemical furfural (2), a possible product from pentoses, instead of petroleum based benzene. Further, we wanted to closely follow as many of the principles of green chemistry as possible. ${ }^{5}$ Specifically we sought to use catalysts in place of stoichiometric reagents, utilize green sources, and limit toxic byproducts.

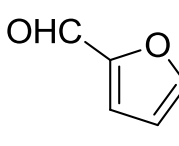

2

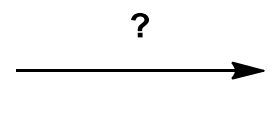

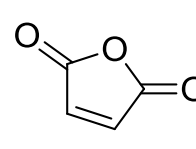
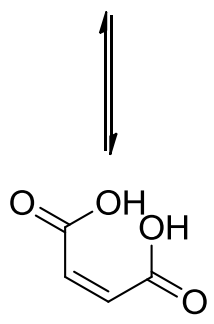

1

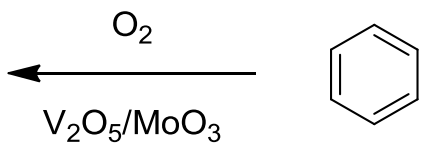

Scheme 2. Industrial synthesis of maleic anhydride and the conversion to its acid (1)

Our interest in hexoses emerged from a desire to synthesize specialty chemicals.

Specifically, we wanted to synthesize the first furan-based ionic liquids. Ionic liquids are a growing area of solvent chemistry but have been known since 1914 when ethylammonium nitrate was synthesized and characterized by Sugden and Wilkins. ${ }^{6}$ Ionic liquids are classified as liquids that consist of ions with melting points lower than $100{ }^{\circ} \mathrm{C} .^{7}$ Their synthetic utility results from high thermal stability, little to no vapor pressure, and tendency to form biphasic systems with organic solvents allowing for ease of recovery. ${ }^{8}$ Ionic liquids have been termed "designer solvents", referring to their versatile physical and chemical properties. Further, ionic liquids 
have been designed to participate in some syntheses, resulting in task-specific ionic liquids. ${ }^{9}$ We sought to design an ionic liquid with a furan scaffold that would allow for further functionalization at the formyl position (Scheme 3).<smiles>Cc1ccc(C=O)o1</smiles>

Scheme 3. Proposed transformation of hexoses to ionic liquids

\section{Results and Discussion}

Our investigation first began by implementing a "green" oxidation of commercially available furfural (2). Singlet oxygen offered an attractive oxidative method that could be catalyzed by a dye sensitizer such as methylene blue. Further, we found reaction conditions that removed the formyl group, resulting in a four carbon scaffold one step away from maleic anhydride (Scheme 4). ${ }^{10}$

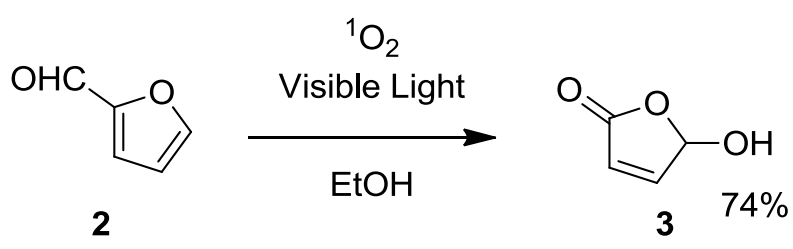

Scheme 4. Oxidation of furfural (2) via singlet oxygen

Intriguingly, $\mathbf{3}$ seemed impervious to further oxidation under singlet or triplet oxygen atmospheres (Table 1, entries 1 and 2). We then attempted manganese(IV) oxide oxidation at 85 ${ }^{\circ} \mathrm{C}$, which resulted in no reaction (Table 1 , entry 3 ). Believing that a stronger oxidant might be needed, and wanting to avoid environmentally unfriendly chromium salts, we attempted the oxidation with 2-iodoxybenzoic acid (IBX). IBX also resulted in returned starting material $\mathbf{3}$ (Table 1, entry 4). Looking into the literature, we found a palladium catalyzed oxidation under 
oxygen atmosphere that looked promising. ${ }^{11}$ In our hands the oxidation resulted in complex reaction mixtures with no evidence of maleic acid (1) or anhydride (Table 1, entry 5).

Table 1. Attempted oxidation of $\mathbf{3}$

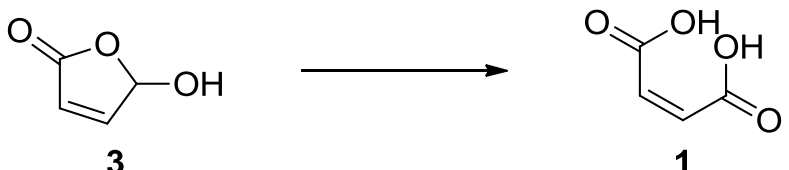

\begin{tabular}{cccc}
\hline Entry & Catalyst & Oxidant & Result \\
1 & Methylene Blue & ${ }^{1} \mathrm{O}_{2}$ & No Rxn \\
2 & None & $\mathrm{O}_{2}$ & No Rxn \\
3 & None & $\mathrm{MnO}_{2}$ & No Rxn \\
4 & None & $\mathrm{IBX}$ & No Rxn \\
5 & ${\mathrm{Pd}(\mathrm{OAc})_{2}}_{6}$ & $\mathrm{O}_{2}$ & Complex Mixtures \\
7 & $\left(\mathrm{NPr}_{4}\right) \mathrm{RuO}_{4}$ & $\mathrm{NMMO}$ & Complex Mixtures \\
& $\mathrm{RuCl}_{3}$ & $\mathrm{NaBrO}_{3}$ & Maleic Acid \\
\hline
\end{tabular}

Returning to the literature, we found references to multiple ruthenium catalyzed oxidations of alcohols to ketones. One such procedure used ruthenium(VII), a powerful oxidant, in the presence of re-oxidant $\mathrm{N}$-methylmorpholine N-oxide (NMMO). ${ }^{12}$ Attempting the oxidation of $\mathbf{3}$ under their conditions gave complex mixtures and again no hint of maleic acid or anhydride (Table 1, entry 6). Undeterred, we tried the oxidation under ruthenium(III) with reoxidant sodium bromate. ${ }^{13}$ Gratifyingly, the oxidation succeeded to give mostly maleic acid (1) with small amounts of its regioisomer, fumaric acid (Table 1, entry 7). To confirm our success, we esterified the acids under standard Fischer esterification conditions and isolated dimethyl maleate and dimethyl fumarate (Scheme 5). 


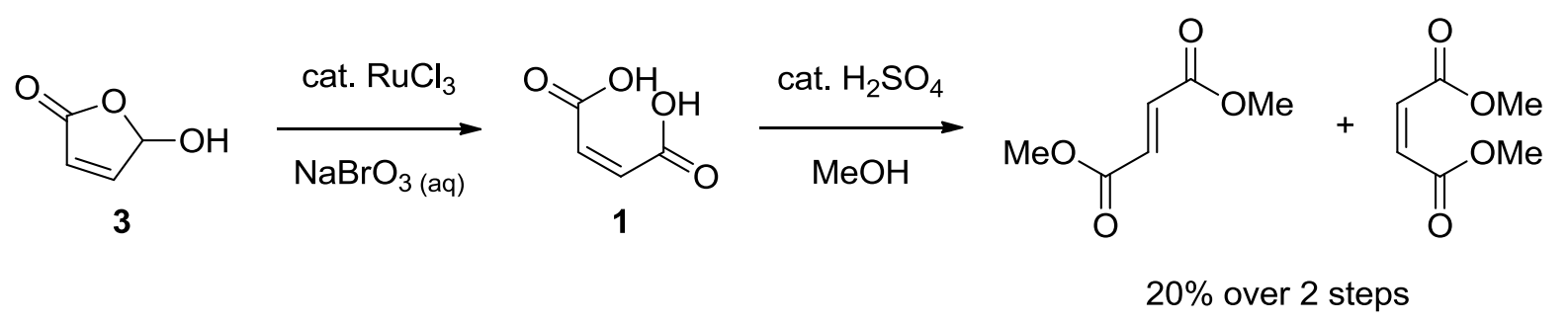

Scheme 5. Conversion of $\mathbf{3}$ to dimethyl maleate/fumarate

With the successful conversion of furfural (2) to maleic acid (1), we turned our attention toward ionic liquids. Building on the concept of platform chemicals, we devised our synthesis based on a compound structurally very similar to HMF: 5-(chloromethyl)furan-2-carbaldehyde, CMF (4). CMF, a possible product from HMF, ${ }^{14}$ is a well known precursor to biofuels (Scheme 6) and has seen emerging utility in the literature. ${ }^{15,16,17}$

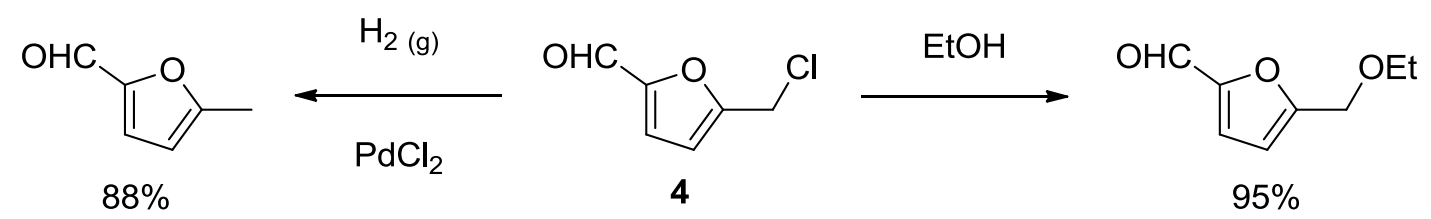

Scheme 6. Conversion of CMF (4) into biofuels ${ }^{15}$

Initially, we successfully formed $\mathbf{4}$ from fructose via a biphasic system of hydrochloric acid and 1,2-dichloroethane prescribed by Mascal and Nikitin. ${ }^{17}$ The reaction proved to be awkward without a continuous extractor, and the work-up was hindersome due to difficulties in separating CMF (4) from the humic byproduct. We found that adding magnesium chloride in a biphasic system cleaned the reaction mixture substantially, as well as increased the yield, and removed the need for continuous extraction (Scheme 7). ${ }^{18}$ With a reliable method of generating 4, we planned to use the electrophilic furfuryl position to react with tertiary amines to synthesize ionic liquids. 

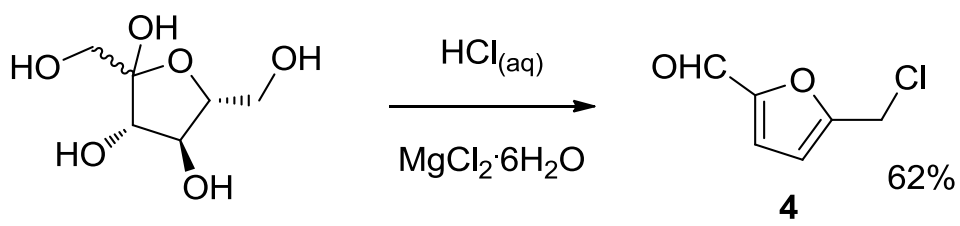

Scheme 7. Conversion of fructose to 4

The reaction of $\mathbf{4}$ with tertiary amines proved to be somewhat problematic. Though we tried various tri-substituted amines, only a handful went in good yields selectively (Table 2). Further, every product proved to be exceedingly hygroscopic, making their handling limited to dry conditions. Of the five successful tertiary amines, only two were liquids at room temperature (Table 2, entries 2 and 3). Entries 4 and 5 have melting points above $200{ }^{\circ} \mathrm{C}$ and therefore technically would not be considered ionic liquids. With two room temperature ionic liquids in hand, we were ready to experiment with the formyl group.

Table 2. Conversion of 4 to ionic liquids 5a-e

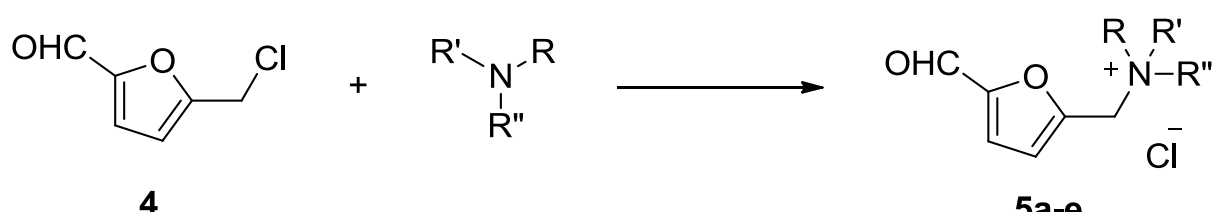

\begin{tabular}{|c|c|c|c|c|c|c|}
\hline Entry & $\mathbf{R}$ & $\mathbf{R}^{\prime}$ & R" & & $\%$ Yield & Mp \\
\hline 1 & Ethyl & Ethyl & Ethyl & $5 \mathbf{a}$ & 96 & $125-134^{\circ} \mathrm{C}$ \\
\hline 2 & Methyl & Methyl & Hexyl & $5 \mathbf{b}$ & 90 & - \\
\hline 3 & Methyl & Methyl & Octyl & $5 c$ & 90 & - \\
\hline 4 & Methyl & \multicolumn{2}{|c|}{$\left(\mathrm{CH}_{2} \mathrm{CH}_{2} \mathrm{CH}_{2} \mathrm{CH}_{2} \mathrm{CH}_{2}\right)$} & $5 d$ & 84 & $>200{ }^{\circ} \mathrm{C}$ \\
\hline 5 & Methyl & \multicolumn{2}{|c|}{$\left(\mathrm{CH}_{2} \mathrm{CH}_{2} \mathrm{OCH}_{2} \mathrm{CH}_{2}\right)$} & $5 e$ & 75 & $>200{ }^{\circ} \mathrm{C}$ \\
\hline
\end{tabular}


During our synthetic efforts toward 5a (Table 2, entry 1), we unexpectedly found an interesting result when our product was analyzed in a methanolic solution. In ${ }^{1} \mathrm{H} \mathrm{NMR}$, the aldehyde peak normally at 9.67 ppm was half the anticipated integration, and two sets of doublets of equal integration appeared alongside the expected set of doublets in the aromatic region. Intrigued, we submitted the sample for low resolution mass spectrum analysis, resulting in peaks at 155, 210, 242, and 256 (Figure 1). The peak at 210 corresponded to the expected product 5a, but the other three dominant peaks were suspect. It dawned on us that the sample had spontaneously reacted with methanol resulting in the dimethyl acetal $6 \mathbf{a}$. The peak at 256 corresponded to the acetal 6a, with 155 being fragmentation via the loss of triethylamine. The peak at 242 corresponded to the hemiacetal. To test our result, we directly reacted $\mathbf{5 a}$ with methanol to form $\mathbf{6 a}$ (Scheme 8). In forming the dimethyl acetal, the ionic liquid also became a liquid at room temperature. This result clearly showed an affinity of the formyl group toward nucleophiles, while offering an explanation to the observed hygroscopicity of compounds 5a-e to form hydrates.

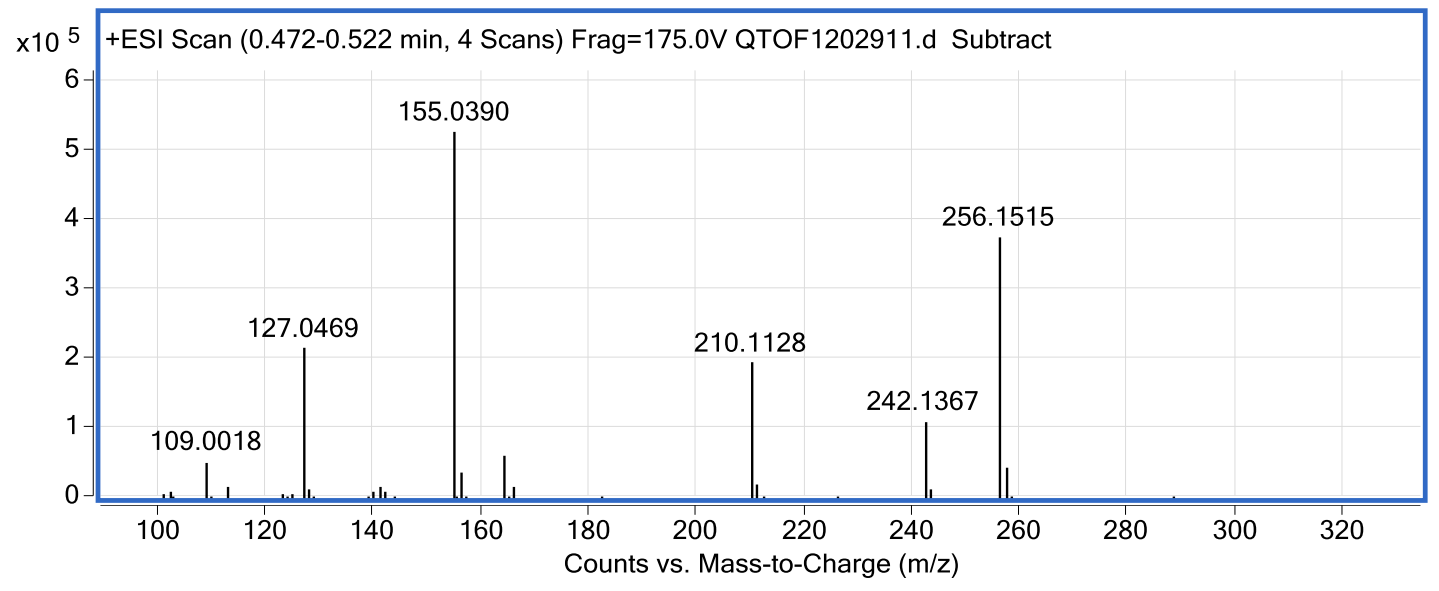

Figure 1. Mass spectrum of 5a in methanol 


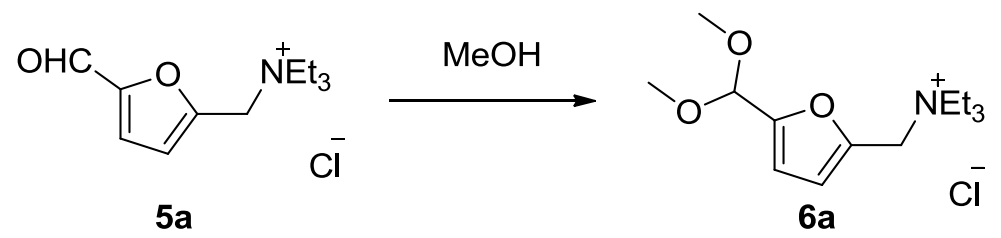

Scheme 8. Reaction of 5a with methanol

To expand on this result, we sought to pair two ionic liquid molecules together through their aldehyde functional groups. We believed molecules such as 7 might lead to ion traps or might be useful surfactants (Scheme 9). Under our reaction conditions we only formed a small portion of 7, while returning some starting 5c. Nevertheless, this result opened the door for further opportunities toward ionic liquid applications and uses.

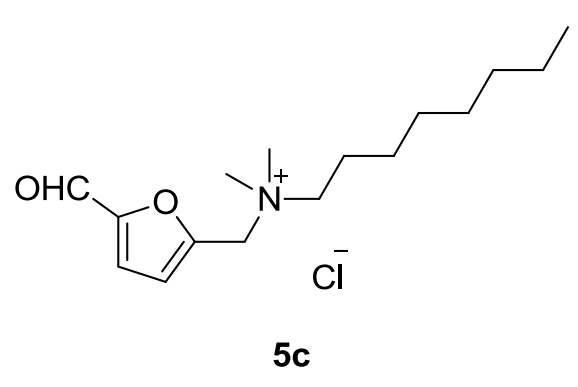

$5 c$
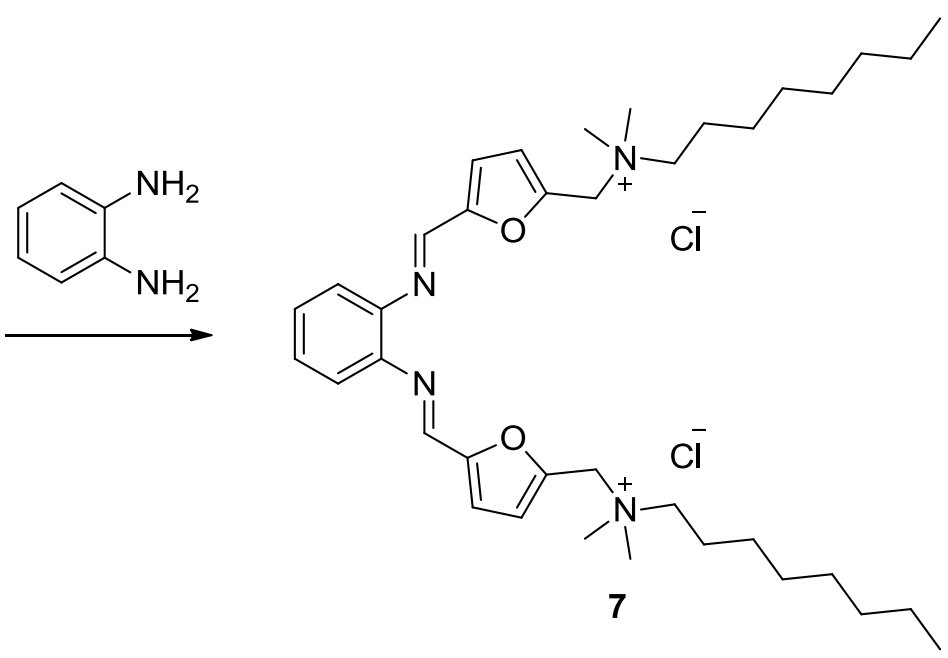

Scheme 9. Formation of 7 from $\mathbf{5 c}$

\section{Conclusion}

The synthesis of maleic acid via biomass was successfully accomplished. Utilizing freely available and environmentally benign singlet oxygen, furfural (2) was easily converted into $\mathbf{3}$ as a stable intermediate toward our goal. Many conditions for the oxidation of $\mathbf{3}$ were attempted, 
but only catalytic ruthenium(III) with stoichiometric sodium bromate proved to successfully give maleic acid (1). Esterification of crude $\mathbf{1}$ gave isolable dimethyl maleate and dimethyl fumarate in modest yields over two steps. We have successfully shown that maleic acid, and ultimately its anhydride, are possible products from "green" transformations. Further research in this area can potentially lead to higher yields of maleic acid or anhydride under optimized oxidative conditions.

We also successfully synthesized the first furan-based ionic liquids. Or synthesis started from renewable fructose and after two steps resulted in two room temperature ionic liquids in good yields. Further, three other ammonium salts were made in good yields over two steps. We have begun preliminary testing of the formyl moiety within our ionic liquids. The formyl group ostensibly is electrophilic enough to condense with alcohols and anilines, as seen in $\mathbf{6 a}$ and $\mathbf{7}$, opening the door to many synthetic possibilities as well as applications. The successful synthesis of bio-based furan ionic liquids presents a renewable method toward specialty chemicals and emerging green chemistries.

\section{Experimental Section}

All NMR spectra were obtained on a Varian VXR spectrometer, operating at 300 or 400 $\mathrm{MHz}$ for ${ }^{1} \mathrm{H} \mathrm{NMR}$ and 75 or $100 \mathrm{MHz}$ for ${ }^{13} \mathrm{C}$ NMR instrument. Chemical shifts in $\mathrm{CDCl}_{3}$ were reported downfield from TMS $(=0 \mathrm{ppm})$ for ${ }^{1} \mathrm{H}$ NMR. Chemical shifts in $\left(\mathrm{CD}_{3}\right)_{2} \mathrm{SO}$ were reported relative to the solvent signal $\left[\left(\mathrm{CD}_{3}\right)_{2} \mathrm{SO}(2.50 \mathrm{ppm})\right]$. Chemical shifts in $\left(\mathrm{CD}_{3}\right)_{2} \mathrm{CO}$ were reported relative to the solvent signal $\left[\left(\mathrm{CD}_{3}\right)_{2} \mathrm{CO}(2.05 \mathrm{ppm})\right]$. All melting points are uncorrected. All reactions were carried out under argon unless otherwise noted. Thin-layer chromatography was performed using commercially available 250 micron silica gel plates 
(Analtech). Preparative thin-layer chromatography was performed using commercially available 1000 micron silica gel plates (Analtech). Visualization of TLC plates was effected with short wavelength ultra violet light $(254 \mathrm{~nm})$. High resolution mass spectra were recorded on an Agilent 6540 QTOF using ESI. All reagents were used directly as obtained commercially unless otherwise noted.

General procedure for the oxidation of furans using singlet oxygen. To a solution of furan $(1 \mathrm{mmol})$ in dry ethanol or 1,2-dichloroethane $(5 \mathrm{~mL})$ was added 10 mole percent of methylene blue. The reaction flask was saturated with oxygen gas for $5-10$ minutes by bubbling the gas through the solution. The reaction flask was placed in a Dewar filled with room temperature water to help keep the solution cool. A 300 Watt incandescent light bulb irradiated the reaction flask for $3-3 \frac{1}{2}$ hours while a continuous stream of oxygen was slowly bubbled through the solution. While irradiating, aluminum foil covered the system from light bulb to Dewar. After every $1 \frac{1 / 2}{2}$ hours, the water in the Dewar was replaced with fresh room temperature water. After TLC showed near completion of the reaction, the reaction was checked for peroxides via acidified starch iodide paper. If positive, dimethylsulfide $(1-2 \mathrm{~mL})$ was added and the reaction was stirred overnight under argon to quench the peroxides. The reaction was tested again for peroxides, which always resulted negative. The solvent was removed under vacuum and the residue was purified via silica gel column chromatography or preparative TLC.

General procedure for the formation of ionic liquids. To a solution of 4 (1 equiv.) in 1,2-dichloroethane $(10 \mathrm{~mL})$ was added tertiary amine $\left(1\right.$ equiv.) at $0{ }^{\circ} \mathrm{C}$. The reaction solution was allowed to warm to room temperature, and then heated to $80{ }^{\circ} \mathrm{C}$ for 3 days. After cooling to room temperature, the solvent was removed under vacuum, and the residue was triturated three 
times with diethyl ether or 1,2-dichloroethane. Very, very hygroscopic! The solid ionic liquids were stored under copious amounts of argon.

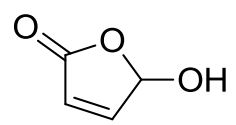

5-Hydroxyfuran-2(5H)-one $3:^{10}$ Prepared using the previously described singlet oxygen procedure in ethanol. Purified via silica gel column chromatography using a mixture of ethyl acetate and hexanes. Yellow solid $(949 \mathrm{mg}, 79 \%) .{ }^{1} \mathrm{H}$ NMR $\left(300 \mathrm{MHz},\left(\mathrm{CD}_{3}\right)_{2} \mathrm{CO}\right): 7.44,(\mathrm{~d}, \mathrm{~J}$ $=6.0 \mathrm{~Hz}, 1 \mathrm{H}), 6.80(\mathrm{br} \mathrm{s}, 1 \mathrm{H}), 6.28(\mathrm{~s}, 1 \mathrm{H}), 6.21(\mathrm{~d}, \mathrm{~J}=6.0 \mathrm{~Hz}, 1 \mathrm{H}) .{ }^{1} \mathrm{H}$ NMR $(400 \mathrm{MHz}$, $\left.\mathrm{CDCl}_{3}\right): 7.33(\mathrm{~d}, \mathrm{~J}=5.6 \mathrm{~Hz}, 1 \mathrm{H}), 6.26(\mathrm{~s}, 1 \mathrm{H}), 6.23(\mathrm{~d}, \mathrm{~J}=6.0 \mathrm{~Hz}, 1 \mathrm{H})$.<smiles>COC(=O)/C=C\C(=O)OC</smiles>

Dimethyl maleate/dimethyl fumarate: ${ }^{13}$ To a solution of $3(159 \mathrm{mg}, 1.6 \mathrm{mmol})$ in acetonitrile $(10.6 \mathrm{~mL})$ was added anhydrous ruthenium(III) chloride $(10 \mathrm{mg}, 0.05 \mathrm{mmol})$, followed by a $0.13 \mathrm{M}$ solution of sodium bromate in water $(16 \mathrm{~mL}, 2.1 \mathrm{mmol})$. The reaction suspension was stirred at room temperature for 24 hours, and then filtered through a silica gel plug. The plug was washed with pure ethanol and the filtrate was concentrated under vacuum to give a crude white/yellow solid. The crude acid (1) was taken into methanol (10 mL) and concentrated sulfuric acid $(0.1 \mathrm{~mL}, 1.8 \mathrm{mmol})$ was added dropwise. The reaction was heated to reflux overnight. After cooling to room temperature, the solvent was removed under vacuum. The oil was dissolved in ethyl acetate and washed twice with saturated $\mathrm{NaHCO}_{3}$, dried over anhydrous $\mathrm{MgSO}_{4}$ and filtered. The solvent was removed under vacuum. The residue was 
purified via silica gel column chromatography using a mixture of ethyl acetate and hexanes. Mixture of cis and trans isomers, colorless oil (45 mg, 20\%). ${ }^{1} \mathrm{H}$ NMR (300 MHz, $\mathrm{CDCl}_{3}$ ): 6.87 (s, 2H), $6.28(\mathrm{~s}, 2 \mathrm{H}), 3.82(\mathrm{~s}, 6 \mathrm{H}), 3.80(\mathrm{~s}, 6 \mathrm{H})$.

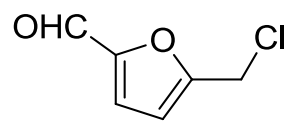

5-(Chloromethyl)furan-2-carbaldehyde (CMF) 4: ${ }^{17,18}$ To a suspension of fructose $(1.4 \mathrm{~g}, 7.6 \mathrm{mmol})$ in toluene $(19 \mathrm{~mL})$ and water $(0.16 \mathrm{~mL})$ was added magnesium chloride hexahydrate $(1.6 \mathrm{~g}, 7.9 \mathrm{mmol})$. The suspension was heated to $75^{\circ} \mathrm{C}$ in air for 30 minutes. Concentrated hydrochloric acid $(2 \mathrm{~mL}, 65 \mathrm{mmol})$ was added dropwise at $75^{\circ} \mathrm{C}$, and the reaction was stirred for 1 hour at $75^{\circ} \mathrm{C}$. After cooling to room temperature, the orange solution was decanted off and the black humic material was washed four times with toluene. The combined organic layers were dried over anhydrous $\mathrm{MgSO}_{4}$, filtered, and concentrated under vacuum. The crude oil was purified via column chromatography using a mixture of ethyl acetate and hexanes. Silica gel contact time was restricted to 20 minutes or less to prevent hydrolysis to HMF. Yellow oil (688 mg, 62\%). ${ }^{1} \mathrm{H}$ NMR (300 MHz, $\left.\mathrm{CDCl}_{3}\right): 9.65(\mathrm{~s}, 1 \mathrm{H}), 7.21(\mathrm{~d}, \mathrm{~J}=3.6 \mathrm{~Hz}, 1 \mathrm{H})$, $6.60(\mathrm{~d}, \mathrm{~J}=3.6 \mathrm{~Hz}, 1 \mathrm{H}), 4.62(\mathrm{~s}, 2 \mathrm{H})$.

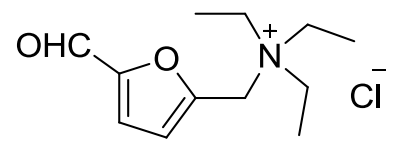

N,N-Diethyl-N-((5-formylfuran-2-yl)methyl)ethanaminium chloride 5a: Prepared using the previously described ionic liquid formation conditions with triethylamine. Triturated with diethyl ether. Brown solid (235 mg, 96\%). Mp: 125-134 ${ }^{\circ} \mathrm{C} .{ }^{1} \mathrm{H}$ NMR (300 MHz, $\mathrm{CDCl}_{3}$ ): $9.68(\mathrm{~s}, 1 \mathrm{H}), 7.46(\mathrm{~d}, \mathrm{~J}=3.6 \mathrm{~Hz}, 1 \mathrm{H}), 7.31(\mathrm{~d}, \mathrm{~J}=3.6 \mathrm{~Hz}, 1 \mathrm{H}), 5.30(\mathrm{~s}, 2 \mathrm{H}), 3.56(\mathrm{q}, \mathrm{J}=7.2 \mathrm{~Hz}$, 
$6 \mathrm{H}), 1.51(\mathrm{t}, \mathrm{J}=7.2 \mathrm{~Hz}, 9 \mathrm{H})$. HRMS ESI $(\mathrm{m} / \mathrm{z})$ : calcd for $\mathrm{C}_{12} \mathrm{H}_{20} \mathrm{NO}_{2}{ }^{+}\left[\mathrm{M}^{+}\right], 210.1489$; found, 210.1488 .

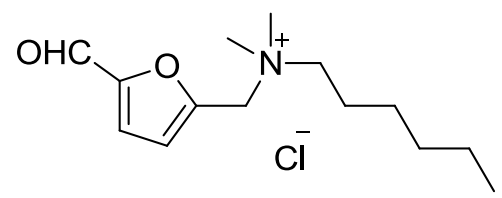

\section{N-((5-Formylfuran-2-yl)methyl)-N,N-dimethylhexan-1-aminium chloride 5b:}

Prepared using the previously described ionic liquid formation conditions with N,N-

dimethylhexan-1-amine. Triturated with diethyl ether. Brown oil (237 mg, 89\%). ${ }^{1} \mathrm{H}$ NMR (300 MHz, $\left.\mathrm{CDCl}_{3}\right): 9.68(\mathrm{~s}, 1 \mathrm{H}), 7.40(\mathrm{~d}, \mathrm{~J}=3.6 \mathrm{~Hz}, 1 \mathrm{H}), 7.31(\mathrm{~d}, \mathrm{~J}=3.6 \mathrm{~Hz}, 1 \mathrm{H}), 5.43(\mathrm{~s}, 2 \mathrm{H})$, 3.50-3.54 (m, 2H), $3.43(\mathrm{~s}, 6 \mathrm{H}), 1.32-1.40(\mathrm{~m}, 8 \mathrm{H}), 0.89-0.92(\mathrm{~m}, 3 \mathrm{H})$. HRMS ESI (m/z): calcd for $\mathrm{C}_{14} \mathrm{H}_{24} \mathrm{NO}_{2}^{+}\left[\mathrm{M}^{+}\right]$, 238.1802; found, 238.1786.

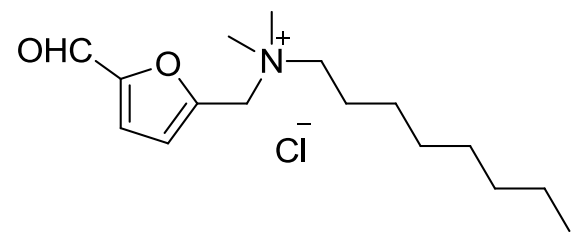

$\mathrm{N}$-((5-Formylfuran-2-yl)methyl)-N,N-dimethyloctan-1-aminium chloride 5c:

Prepared using the previously described ionic liquid formation conditions with $\mathrm{N}, \mathrm{N}$ -

dimethyloctan-1-amine. Triturated with diethyl ether. Brown oil (272 mg, 90\%). ${ }^{1} \mathrm{H}$ NMR (300 $\left.\mathrm{MHz}, \mathrm{CDCl}_{3}\right): 9.68(\mathrm{~s}, 1 \mathrm{H}), 7.39(\mathrm{~d}, \mathrm{~J}=3.6 \mathrm{~Hz}, 1 \mathrm{H}), 7.31(\mathrm{~d}, \mathrm{~J}=3.6 \mathrm{~Hz}, 1 \mathrm{H}), 5.43(\mathrm{~s}, 2 \mathrm{H}), 3.50-$ $3.54(\mathrm{~m}, 2 \mathrm{H}), 3.44(\mathrm{~s}, 6 \mathrm{H}), 1.25-1.36(\mathrm{~m}, 12 \mathrm{H}), 0.86-0.90(\mathrm{~m}, 3 \mathrm{H})$. HRMS ESI (m/z): calcd for $\mathrm{C}_{16} \mathrm{H}_{28} \mathrm{NO}_{2}^{+}\left[\mathrm{M}^{+}\right], 266.2115$; found, 266.2099.

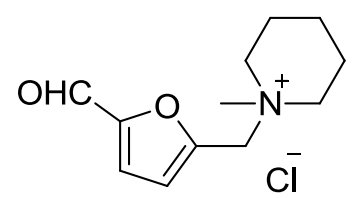


1-((5-formylfuran-2-yl)methyl)-1-methylpiperidin-1-ium chloride 5d: Prepared using the previously described ionic liquid formation conditions with $\mathrm{N}$-methylpiperidine. Triturated with 1,2-dichloroethane. Light brown solid (202 mg, 84\%). Mp: > $200{ }^{\circ} \mathrm{C} .{ }^{1} \mathrm{H}$ NMR (300 $\left.\mathrm{MHz}, \mathrm{CDCl}_{3}\right): 9.68(\mathrm{~s}, 1 \mathrm{H}), 7.42(\mathrm{~d}, \mathrm{~J}=3.6 \mathrm{~Hz}, 1 \mathrm{H}), 7.30(\mathrm{~d}, \mathrm{~J}=3.6 \mathrm{~Hz}, 1 \mathrm{H}), 5.51(\mathrm{~s}, 2 \mathrm{H}), 3.80-$ $3.88(\mathrm{~m}, 4 \mathrm{H}), 3.39(\mathrm{~s}, 3 \mathrm{H}), 1.74-2.01(\mathrm{~m}, 6 \mathrm{H})$. HRMS ESI $(\mathrm{m} / \mathrm{z})$ : calcd for $\mathrm{C}_{12} \mathrm{H}_{18} \mathrm{NO}_{2}^{+}\left[\mathrm{M}^{+}\right]$, 208.1332; found, 208.1324.

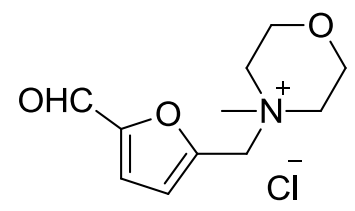

4-((5-Formylfuran-2-yl)methyl)-4-methylmorpholin-4-ium chloride 5e: Prepared using the previously described ionic liquid formation conditions with N-methylmorpholine. Triturated with 1,2-dichloroethane. Light brown solid (185 mg, 75\%). Mp: $>200{ }^{\circ} \mathrm{C}$. ${ }^{1} \mathrm{H}$ NMR (300 MHz, $\left.\left(\mathrm{CD}_{3}\right)_{2} \mathrm{SO}\right): 9.69(\mathrm{~s}, 1 \mathrm{H}), 7.64(\mathrm{~d}, \mathrm{~J}=3.3 \mathrm{~Hz}, 1 \mathrm{H}), 7.15(\mathrm{~d}, \mathrm{~J}=3.6 \mathrm{~Hz}, 1 \mathrm{H}), 5.04(\mathrm{~s}$, 2H), 3.90-4.01 (m, 4H), 3.42-3.56 (m, 4H), 3.19 (s, 3H). HRMS ESI (m/z): calcd for $\mathrm{C}_{11} \mathrm{H}_{16} \mathrm{NO}_{3}^{+}\left[\mathrm{M}^{+}\right], 210.1125$; found, 210.1119 .

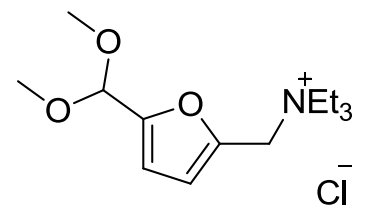

N-((5-(dimethoxymethyl)furan-2-yl)methyl)-N,N-diethylethanaminium chloride 6a: To 5a (103.3 mg, $0.42 \mathrm{mmol})$ was added methanol $(0.07 \mathrm{~mL}, 1.7 \mathrm{mmol})$. The reaction was heated to $40{ }^{\circ} \mathrm{C}$ and stirred overnight. The excess solvent was removed under vacuum, yielding 6a as a crude brown oil. MS ESI (m/z): 155.0, 210.1 (5a), 242.1, 256.2 (6a). 


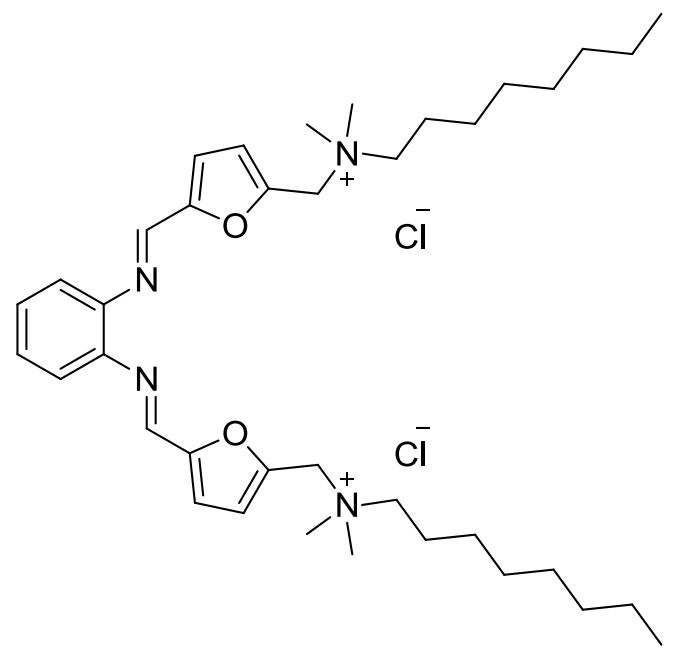

N,N'-((5,5'-((1E,1'E)-(1,2-phenylenebis(azanylylidene))bis(methanylylidene)) bis(furan-5,2-diyl))bis(methylene) $)$ bis(N,N-dimethyloctan-1-aminium) chloride 7: ${ }^{19}$ To a solution of 5c (311 mg, $1.0 \mathrm{mmol})$ in 1,2-dichloroethane $(10 \mathrm{~mL})$ was added phenylenediamine (56 mg, $0.52 \mathrm{mmol}$ ). The reaction was stirred overnight at room temperature, and analyzed directly afterword. MS ESI (m/z): 266.2 (5c), 302.2 (7).

\section{References}

1. (a) Rosatella, A. A.; Simeonov, S. P.; Frade, R. F. M.; Afonso, C. A. M. Green Chem. 2011, 13, 754-793. (b) Serrano-Ruiz, J. C.; West, R. M.; Dumesic, J. A. Annu. Rev. Chem. Biomol. Eng. 2010, 1, 79-100.

2. (a) Crisci, A. J.; Tucker, M. H., Dumesic, J. A.; Scott, S. L. Top. Catal. 2010, 53, 11851192. (b) Zhao, H.; Holladay, J. E.; Brown, H.; Zhang, Z. C. Science, 2007, 316, $1597-$ 1600. (c) Bicker, M.; Kaiser, D.; Ott, L.; Vogel, H. J. Supercrit. Fluids 2005, 36, 118126.

3. Lohbeck, K.; Haferkorn, H.; Fuhrmann, W.; Fedtke, N. Maleic and Fumaric Acids Ullman's Encyclopedia of Industrial Chemistry 2000, 22, 145-155.

4. (a) Diels, O.; Alder, K. Liebigs Ann. Chem. 1931, 490, 267-276. (b) Kloetzel, M. C. The Diels-Alder Reaction with Maleic Anhydride. Organic Reactions, 2011, 1-59.

5. Anastas, P. T.; Warner, J. C. Green Chemistry: Theory and Practice, (Oxford University Press: New York) 1998, 30.

6. Sugden, S.; Wilkins, H. J. Chem. Soc. 1929, 1291-1298. 
7. Wasserscheid, P.; Keim, W. Angew. Chem. Int. Ed. 2000, 39, 3772-3789.

8. Werner, S.; Haumann, M.; Wasserscheid, P. Annu. Rev. Chem. Biomol. Eng. 2010, 1, 203-230.

9. (a) Cole, A. C.; Jensen, J. L.; Ntai, I.; Tran, L. T.; Weaver, K. J.; Forbes, D. C.; Davis Jr.; J. H. J. Am. Chem. Soc. 2002, 124, 5962-5963. (b) Amarasekara, A. S.; Owereh, O. S. Ind. Eng. Chem. Res. 2009, 48, 10152-10155. (c) Kraus, G. A.; Guney, T. Green Chemistry 2012, 14, 1593-1596.

10. Cottier, L.; Descotes, G.; Nigay, H.; Parron, J.-C.; Grégoire, V. Bull. Soc. Chim. Fr. 1986, 844-850.

11. Nishimura, T.; Onoue, T.; Ohe, K.; Uemura, S. J. Org. Chem. 1999, 64, 6750-6755.

12. Maier, C. A.; Wunsch, B. J. Med. Chem. 2002, 45, 438-448.

13. Chih-Yu Chen, A.; Laliberte, S.; Larouche, G.; Han, Y.; McKay, D. PCT Int. Appl. 2011, WO 2011020193 A1 20110224.

14. Sanda, K.; Rigal, L.; Gaset, A. Carbohydr. Res. 1989, 187, 15-23.

15. Mascal, M.; Nikitin, E. B. Angew. Chem. 2008, 120, 8042-8044; Angew. Chem. Int. Ed. 2008, 47, 7924-7926.

16. Mascal, M.; Nikitin, E. B. ChemSusChem 2009, 2, 423-426.

17. Mascal, M.; Nikitin, E. B. ChemSusChem 2009, 2, 859-861.

18. (a) Quiroz-Florentino, H.; Hernández-Benitez, R. I.; Aviña, J. A.; Burgueño-Tapia, E.; Tamariz, J. Synthesis, 2011, 1106-1112. (b) Hamada, K.; Yoshihara, H.; Suzukamo, G. Chem. Lett. 1982, 617-618.

19. Mukhopadhyay, M.; Reddy, M. M.; Maikap, G. C.; Iqbal, J. J. Org. Chem. 1995, 60, 2670-2676. 


\section{Chapter 3. REAL-TIME IMAGING OF PROMOTER ACTIVITY TO}

\section{MONITOR GENE EXPRESSION}

\section{Introduction}

Tyrosine protein kinases play central roles in communication within and between cells and have been implicated in cancer proliferation. ${ }^{1}$ Drugs that can control tyrosine kinases and prevent proliferation offer attractive solutions for cancer patients. ${ }^{2}$ One such drug, Imatinib (Figure 1), historically has been used to treat chronic myelogenous leukemia and other cancers. ${ }^{3}$ Unfortunately, recent studies show resistance in cancer patients, so new drugs are needed to actively inhibit these kinases. ${ }^{4}$

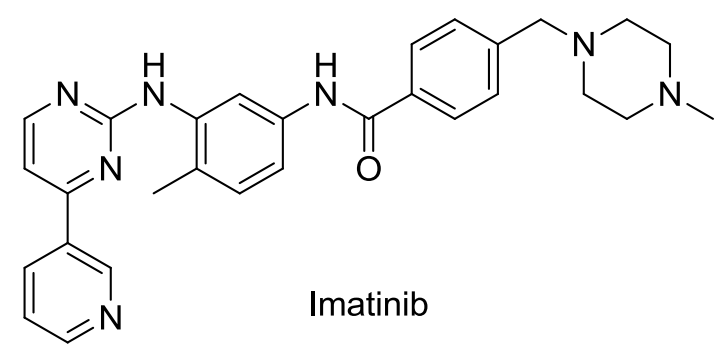

Figure 1. Structure of Imatinib

The Parke-Davis Company developed a class of novel inhibiters of Abelson kinase, which is often up-regulated in cancer. ${ }^{5}$ One such inhibitor, PD173955 (Figure 2), contains a substituted pyrido[2,3- $d]$ pyrimidine system, which is known to be present in potent tyrosine kinase inhibitors. ${ }^{6}$ Although PD173955 showed excellent inhibition of cancer proliferation, ${ }^{3,}$, 8 it has poor water solubility, complicating its use as a therapeutic. Further, it also showed toxicity to normal cells via its ability to inhibit other kinases other than Abelson tyrosine kinase. ${ }^{9}$ To circumvent the solubility issue present in PD173955, as well as its excessive inhibition, the Kraus and Nilsen-Hamilton groups sought to alter its structure while maintaining the key 
pyrido[2,3- $d]$ pyrimidine feature. ${ }^{10}$ They found that by introducing an extra carbon unit within the structure reduced kinase inhibition by a factor of 200. Also, they noted that introduction of an amino group where a thiomethyl group was in PD173955 increased water solubility. The new compound, 1, noted as amino-PDC (Figure 2), was then slated for imaging of gene expression.

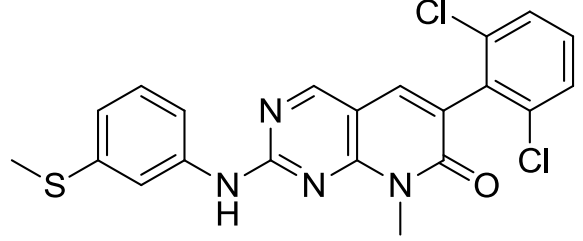

PD173955<smiles>Cn1c(=O)c(-c2c(Cl)cccc2Cl)cc2cnc(NCc3cccc(N)c3)nc21</smiles>

amino-PDC (1)

Figure 2. PD173955 and amino-PDC

Changes in gene expression are central to most alterations in cellular functions, yet our current means of measuring transcriptional changes in real time are limited. ${ }^{11}$ Currently all reporter systems inherently exhibit time-lag between transcription and observation. ${ }^{12}$ RNA reporter systems will aid in decreasing the time between transcription and observation. One type of RNA reporter system that has seen some success in living cells is the green fluorescent protein. ${ }^{13}$ The fluorophore encased in green fluorescent protein that enables fluorescence is 4hydroxybenzylidene imidazolinone (HBI) (Figure 3). ${ }^{14}$ Recently, Jaffrey and coworkers generated RNA apatmers that bind various green fluorescent protein mimics of HBI. ${ }^{15}$ The RNA-fluorophore complex, denoted as Spinach RNA, creates a signal by promoting fluorescence in the specific green fluorescent protein mimic DFHBI (Figure 3). We sought to test this green fluorescent protein mimic against our own developed dye labeled ligands, which incorporate amino-PDC or aminoglycoside. Unlike Jaffrey and coworkers, we sought to visualize gene expression through FRET on much less abundant mRNA. Our system utilized a 
string of repeating RNA aptamers that bind our fluorescent ligands and visualize recognition via FRET.<smiles>[Y]C1=N/C(=C\c2ccc(O)cc2)C(=O)N1[Y]</smiles>

HBI<smiles>CC1=N/C(=C\c2cc(F)c(O)c(F)c2)C(=O)N1C</smiles>

DFHBI (2)

Figure 3. $\mathrm{HBI}$ and DFHBI

FRET, Förster resonance energy transfer, also known as fluorescence resonance energy transfer, is a mechanism of energy transfer from one fluorophore to another. ${ }^{16}$ The mechanism of transfer occurs when the donor, in its excited state, transfers energy non-radiatively to a neighboring molecule, the acceptor, in its ground state via dipole-dipole interactions. ${ }^{17}$ FRET is advantageous because it only occurs over $1-10 \mathrm{~nm}$ distances, allowing scientists to discover whether two molecules are in close proximity. ${ }^{17,} 18$ We planned to implement FRET in our system by exciting the donor which subsequently would transfer energy to the neighboring acceptor chemically bond to the ligand on the aptamer chain. Successful aptamer synthesis would be indicated by FRET visualization.

\section{Results and Discussion}

As chemists, our main task was to provide dye labeled ligands for biological testing. Our group had already published a synthesis of $\mathbf{1},{ }^{10}$ yet some hurdles needed to be solved. First, nucleophilic addition of 3-aminobenzylamine to the pyrimidine sulfone needed to be optimized. Second, the addition of the glycine group needed reworking to ensure reproducibility. Finally, we needed to attach dyes to the ligand to allow FRET within the engineered yeast cells. We tackled the nucleophilic addition first. 
The synthesis of the core backbone of amino-PDC began from commercially available 3 (Scheme 1). The synthesis proceeded smoothly until the potassium fluoride (KF) cyclization step. Compound $\mathbf{8}$ proved to be insoluble in most organic solvents, and could not be purified by silica gel column or TLC. In my hands the prescribed recrystallization worked modestly, and further crystallizations from the mother liquor could certainly give even greater yields. Nevertheless, with $\mathbf{8}$ in hand, we were ready to attempt the oxidation and nucleophilic aromatic substitution to produce amino-PDC (1).
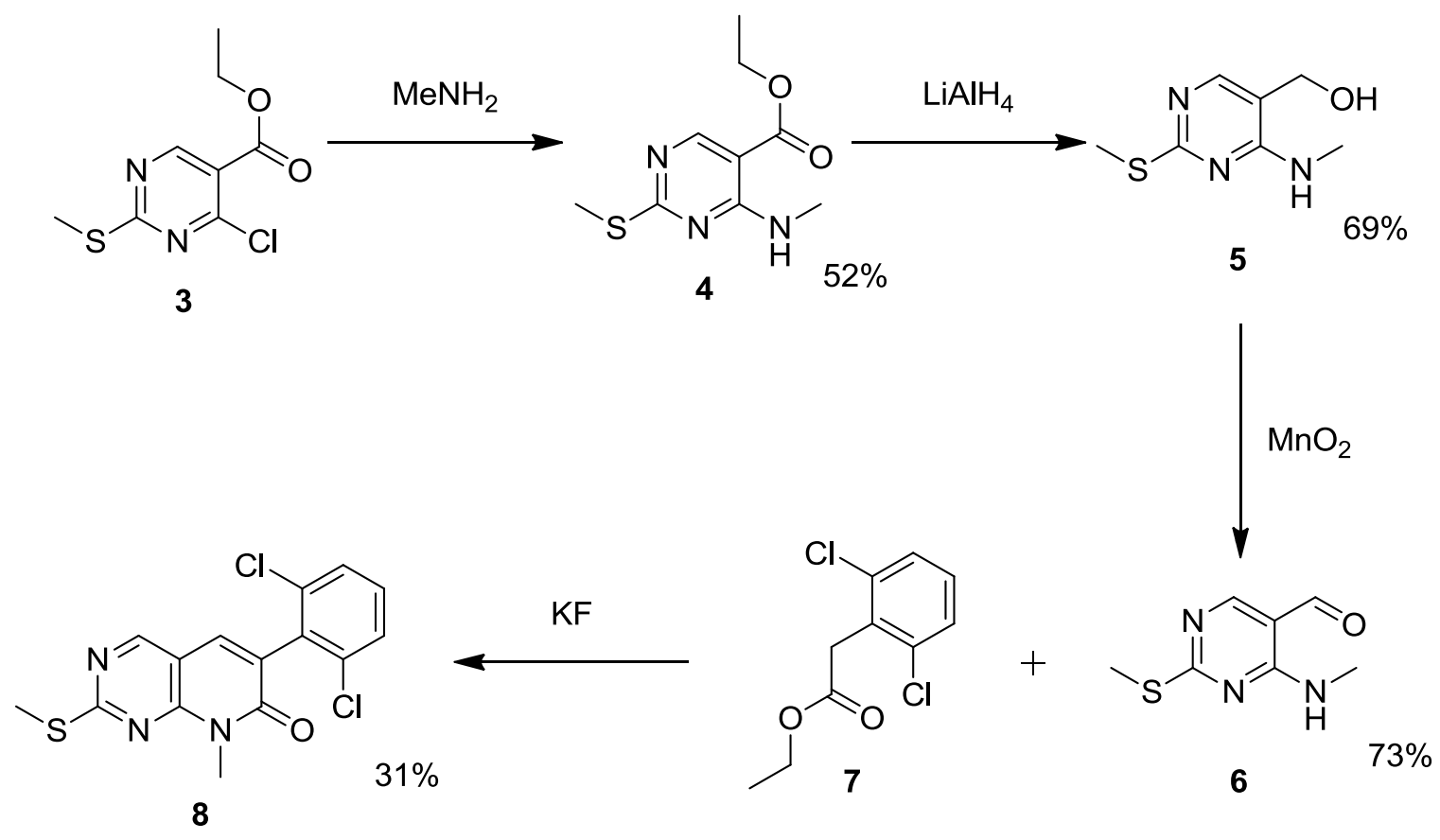

Scheme 1. Synthesis of $\mathbf{8}$ from commercially available $\mathbf{3}$

Interestingly, the oxidation of $\mathbf{8}$ with meta-chloroperbenzoic acid (mCPBA) gave quantitative yields of 9 compared to $87 \%$ as reported (Scheme 2). ${ }^{10}$ However, the nucleophilic aromatic substitution step gave complex mixtures. After extensive purification and various attempted reaction conditions, we found the published reaction conditions of heating N,Ndimethylformamide (DMF) to reflux overnight actually yielded two compounds of similar $\mathrm{R}_{\mathrm{f}}$ : 
Amino-PDC (1) and a slightly more polar substrate with a similar ${ }^{1} \mathrm{H}$ NMR. Upon obtaining a low resolution mass spectrum of the unknown compound, we realized it was a formylated form of $1(\mathrm{~m} / \mathrm{z}=454)$. We hypothesized this reaction to occur via nucleophilic attack of formed amino-PDC on excess DMF to yield formylated amino-PDC and N,N-dimethylamine. To prevent this formylation reaction from occurring, we lowered the temperature of the reaction from reflux to $150{ }^{\circ} \mathrm{C}$, just below the boiling point of DMF. This resulted in far less formylated amino-PDC and increased the yield of amino-PDC (1) to $82 \%$, compared to the published $61 \% .^{10}$ We also noticed that 3-aminobenzylamine supplied by Sigma-Aldrich ${ }^{\circledR}$ was tantamount for success. Trials of 3-aminobenzylamine supplied by TCI America ${ }^{\circledR}$ resulted in very complex mixtures and exceedingly low yields of amino-PDC.

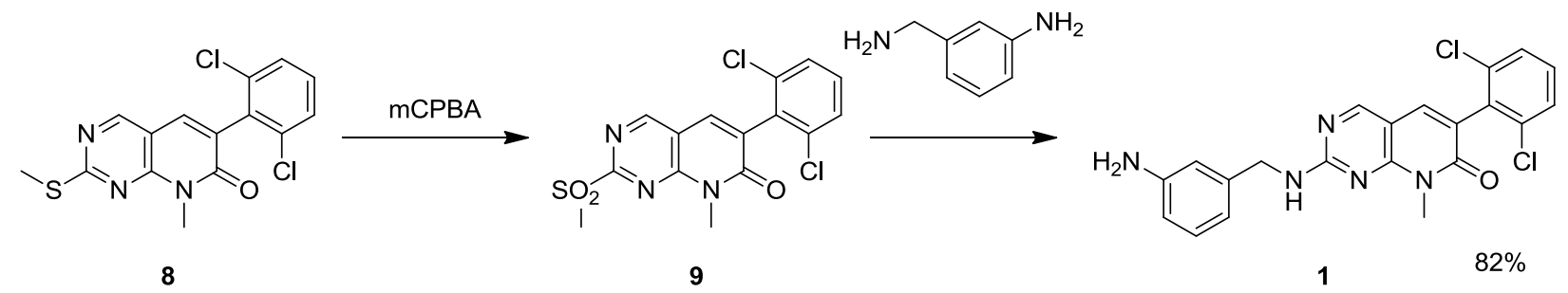

Scheme 2. Completed synthesis of amino-PDC (1)

With the synthesis of amino-PDC (1) streamlined, we were prepared to tackle the addition of glycine. We reasoned the coupling of N-hydroxysuccinimidyl esters (NHS esters) would be selective toward unhindered, alkyl primary amines over hindered, aryl, and/or secondary amines as found in $\mathbf{1}$. The glycine subunit was needed to provide an unhindered, alkyl primary amine extender for successful coupling with dyes in their NHS ester form. Further, we wanted an extender that would have minimal interactions with the aptamer binding site while keeping the dye portion relatively close to the ligand to ensure FRET across aptamer loops. Without the glycine linker, we found the dye coupling reactions with amino-PDC to be low yielding and gave complex mixtures. 
Previously, PDC-Gly (10) was synthesized from bromoacetyl bromide, followed by amination with ammonia gas (Scheme 3). ${ }^{10}$ Any attempts at PDC-Gly (10) through this method failed, so a new, reproducible method was sought. Reasoning that bromoacetyl bromide was too reactive and unstable during storage, we sought to use a milder amidation procedure (Scheme 4). ${ }^{19}$ This method was also advantageous because N-Boc-glycine proved to be more stable than bromoacetyl bromide. Also, deprotection of the Boc group was easily accomplished using $3 \mathrm{~N}$ $\mathrm{HCl},{ }^{19}$ which inherently was easier to handle than gaseous ammonia. Further, only an $8 \%$ difference in yield was noticed between employing Steglich amidation over bromoacetyl bromide acylation.<smiles>Cn1c(=O)c(-c2c(Cl)cccc2Cl)cc2cnc(NCc3cccc(N)c3)nc21</smiles>

1

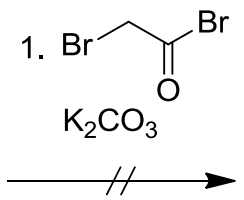

2. $\mathrm{NH}_{3}$<smiles>Cn1c(=O)c(-c2c(Cl)cccc2Cl)cc2cnc(NCc3cccc(NC(=O)CN)c3)nc21</smiles>

10

Scheme 3. Initial synthesis of PDC-Gly (10)

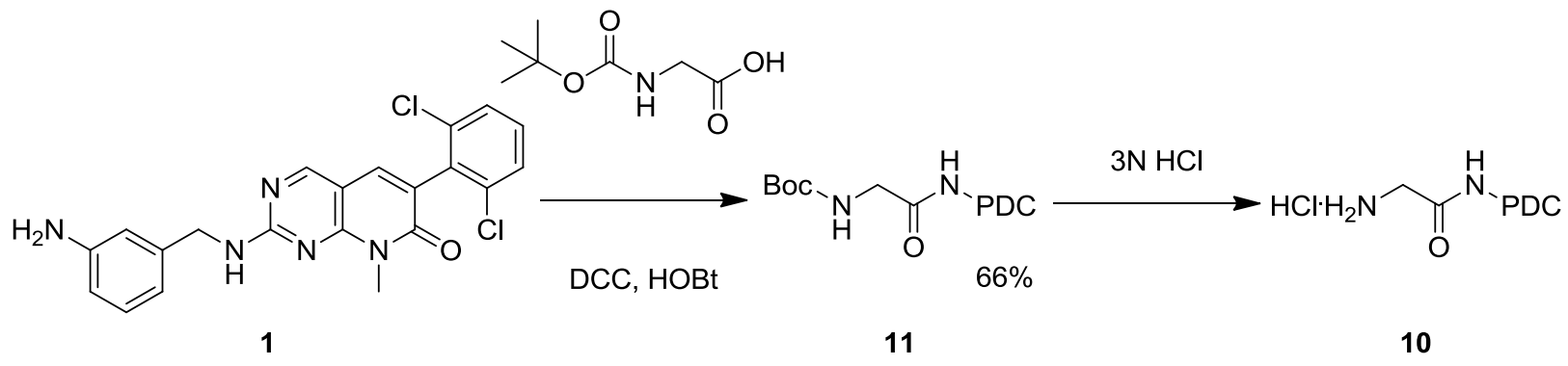

Scheme 4. Alternative synthesis of PDC-Gly (10) as its $\mathrm{HCl}$ salt

Once we successfully developed a reproducible procedure for the synthesis of PDC-

Gly $\mathrm{HCl}$, we were then ready to attach cyanine dyes to generate the desired FRET pair.

Employing the synthetic utility of NHS esters and their preferential affinity toward unhindered, 
primary alkyl amines, we developed a procedure that allowed for simple coupling of PDC-Gly to cyanine dyes. Our collaborators determined that Cy3 and Cy5 (Figure 4) formed a suitable FRET pair; where $\mathrm{Cy} 3$ would act as the donor and Cy5 as the accepter. Additionally, both were commercially available as their respective NHS esters. Coupling PDC-Gly with Cy5 and Cy3 gave dye bound ligands 12 and $\mathbf{1 3}$ for use in cellular imaging (Scheme 5).

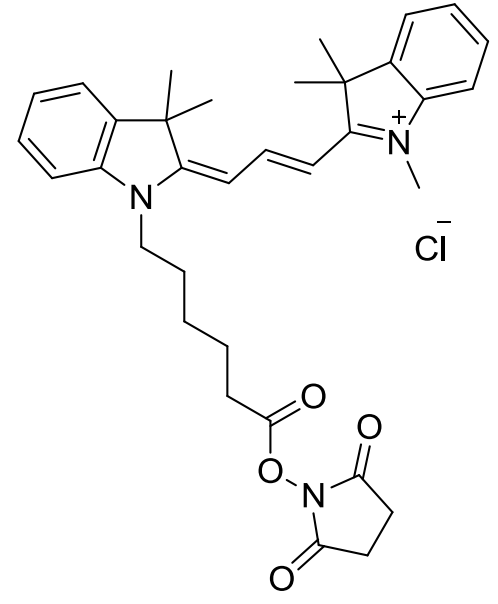

Cy3 NHS

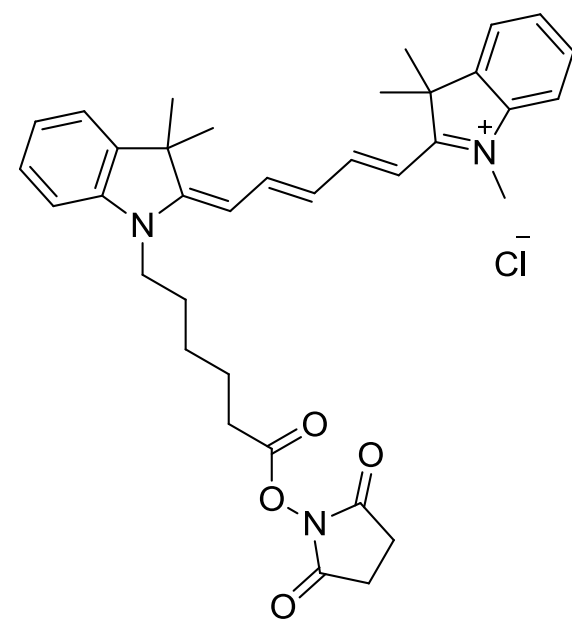

Cy5 NHS

Figure 4. Commercially available cyanine dyes

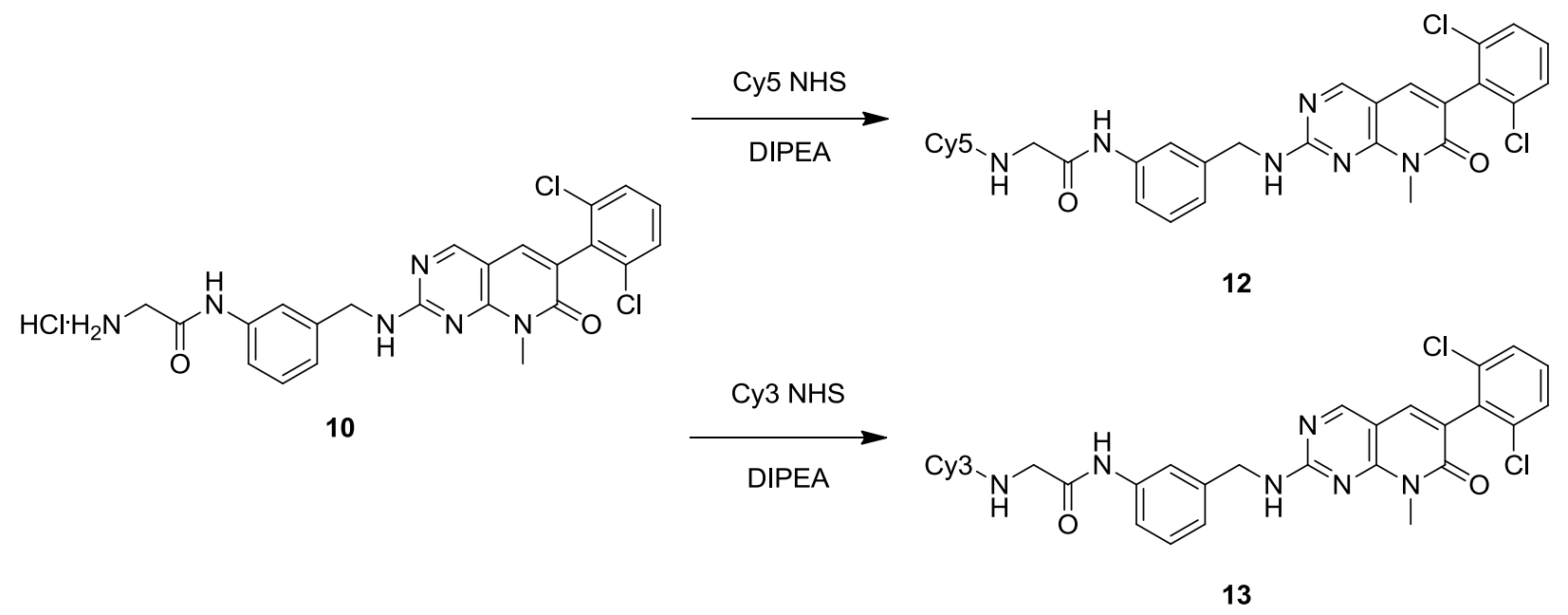

Scheme 5. Synthesis of FRET pair Cy5-PDC-Gly (12) and Cy3-PDC-Gly (13) 
The biological experiment was designed such that once gene expression occurred, the RNA aptamer chain would be synthesized in vivo by the engineered yeast cell. Each sequential aptamer within the chain was identical and selected based on its affinity toward the ligand, PDC. Once the aptamer chain was synthesized, the dye bound ligands would attach themselves to the aptamer loops. When Cy5-PDC-Gly (12) and Cy3-PDC-Gly (13), a FRET pair, bound within

close proximity an increase in the FRET signal was noticed. ${ }^{11}$ This increase in FRET confirmed successful synthesis of the aptamer chain and ligand binding. Therefore, gene expression was visualized in real time.

To test the biological utility of this result, we sought to try other ligands with suitable aptamers. Aminoglycosides tobramycin and paromomycin sulfate (Figure 5) were chosen and appropriate aptamers were selected based on affinity toward each. Unlike PDC-Gly (10), both tobramycin and paromomycin sulfate were commercially available. Further, they inherently each have one primary, unhindered alkyl amine. Therefore they could be reacted directly with the cyanine dye NHS esters.<smiles>NC[C@H]1O[C@H](O[C@@H]2[C@@H](N)C[C@@H](N)[C@H](O[C@@H]3O[C@H](CO)[C@@H](O)[C@H](N)[C@H]3O)[C@H]2O)[C@@H](N)C[C@H]1O</smiles>

Tobramycin

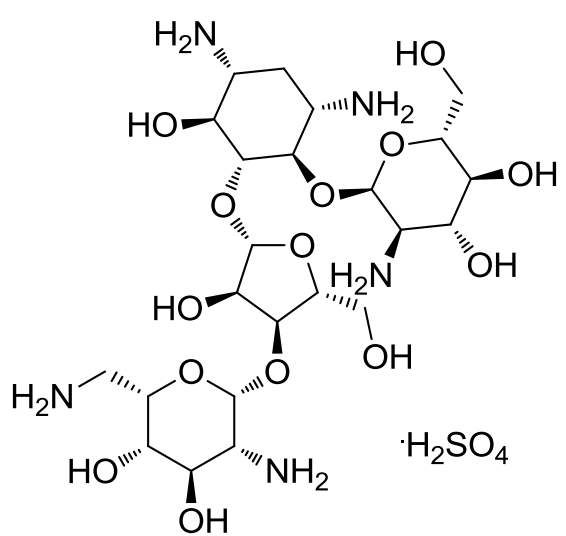

Paromomycin Sulfate

Figure 5. Tobramycin and paromomycin sulfate 
New dyes were selected as well due to the poor solubility of paromomycin sulfate in dimethylsulfoxide (DMSO). Fortunately water soluble sulfonated forms of Cy3 and Cy5 NHS esters were also commercially available (Figure 6), allowing for the coupling reaction with paromomycin to be run under aqueous conditions. ${ }^{20}$ Tobramycin was mildly soluble in dimethylsulfoxide, so it was coupled under similar conditions as PDC-Gly. Similar to the PDC series, the dye bound aminoglycoside FRET pair $\mathbf{1 4}$ and 15, as well as FRET pair 16 and 17, showed increases in FRET for their respective systems (Figure 7). ${ }^{11}$

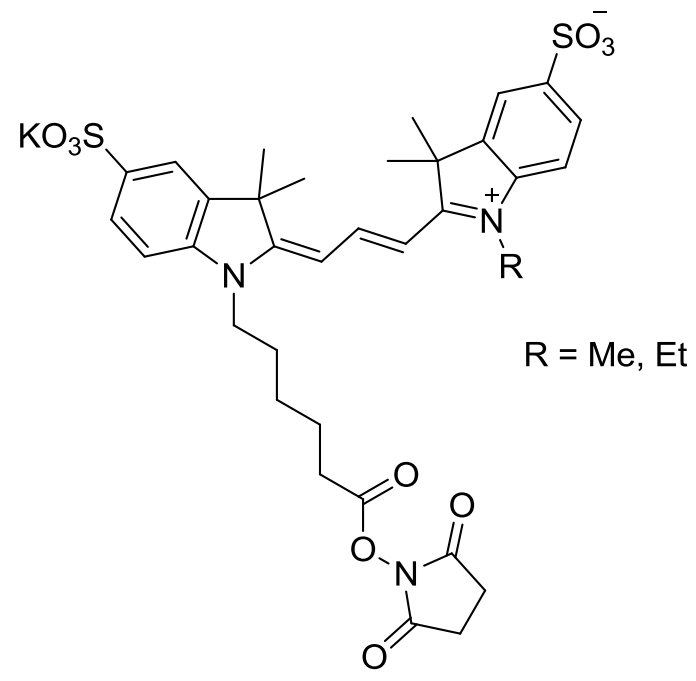

Cy3- $\mathrm{SO}_{3} \mathrm{NHS}$

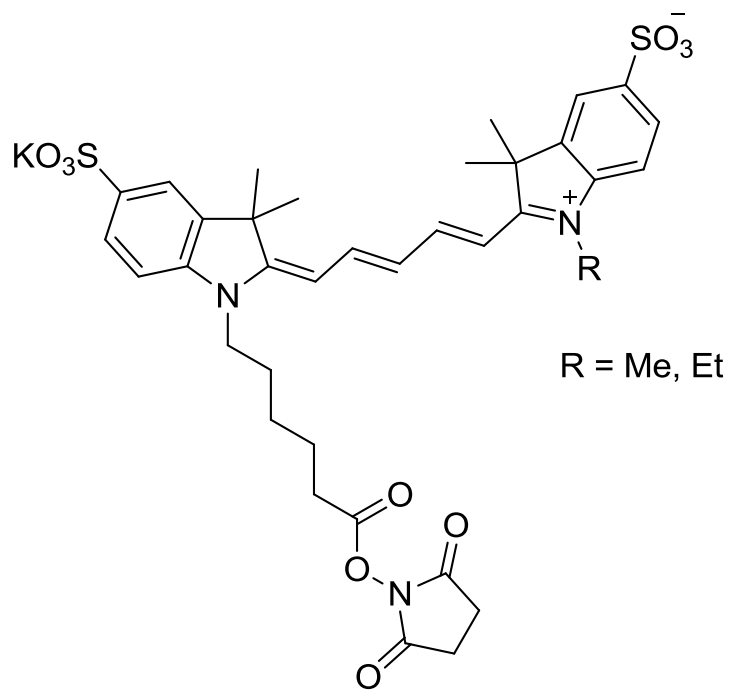

Cy5 $-\mathrm{SO}_{3} \mathrm{NHS}$

Figure 6. Commercially available sulfonated cyanine dyes 


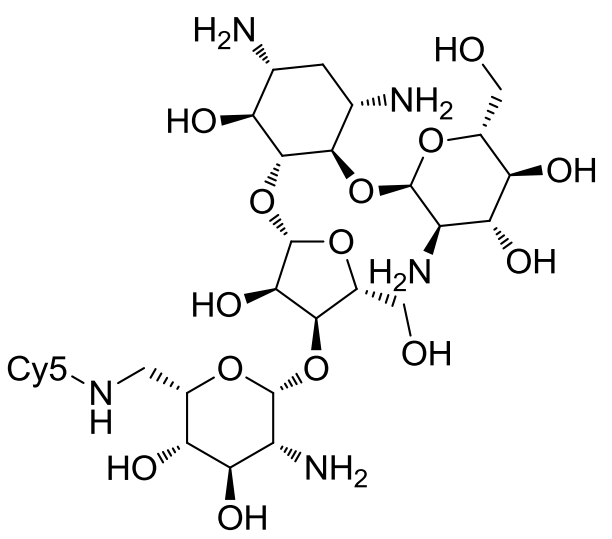

14<smiles>N[C@H]1C[C@@H](O)[C@@H](CNC2CCCCC2)O[C@H]1O[C@@H]1[C@@H](N)C[C@@H](N)[C@H](O[C@@H]2O[C@H](CO)[C@@H](O)[C@H](N)[C@H]2O)[C@H]1O</smiles>

16

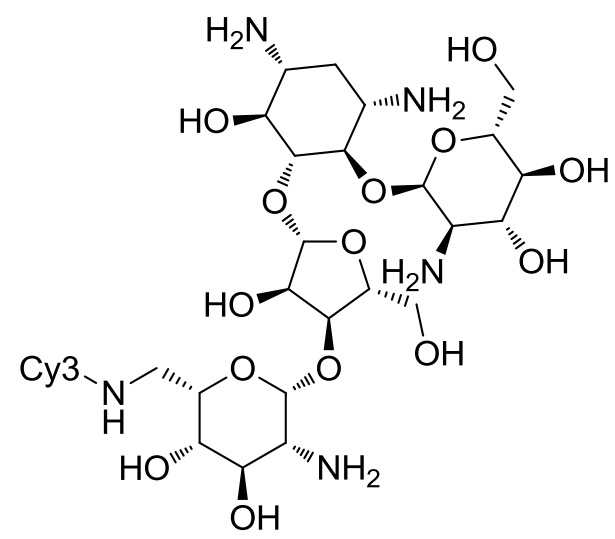

15

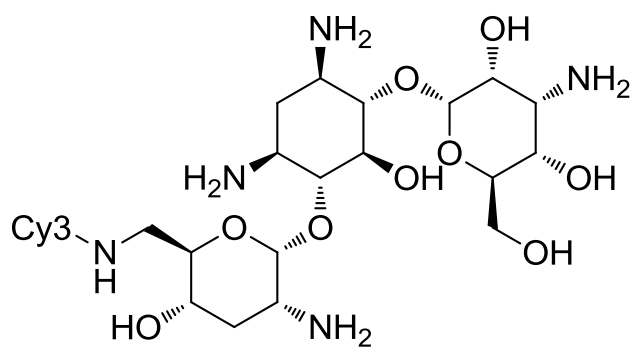

17

Figure 7. Aminoglycoside FRET pairs

With these results in hand, we sought to determine whether the Spinach RNA tag (DFHBI) would also show transcription of mRNA. DFHBI was synthesized according to Jaffrey and coworkers (Scheme 6). ${ }^{15}$ We found the order of addition between methylamine and potassium carbonate to be crucial for reaction success. If potassium carbonate was added before methylamine, then no reaction occurred. Therefore it was critical that methylamine be added before potassium carbonate to produce DFHBI. When testing DFHBI in our yeast system, no increase in signal was noticed beyond the background. 


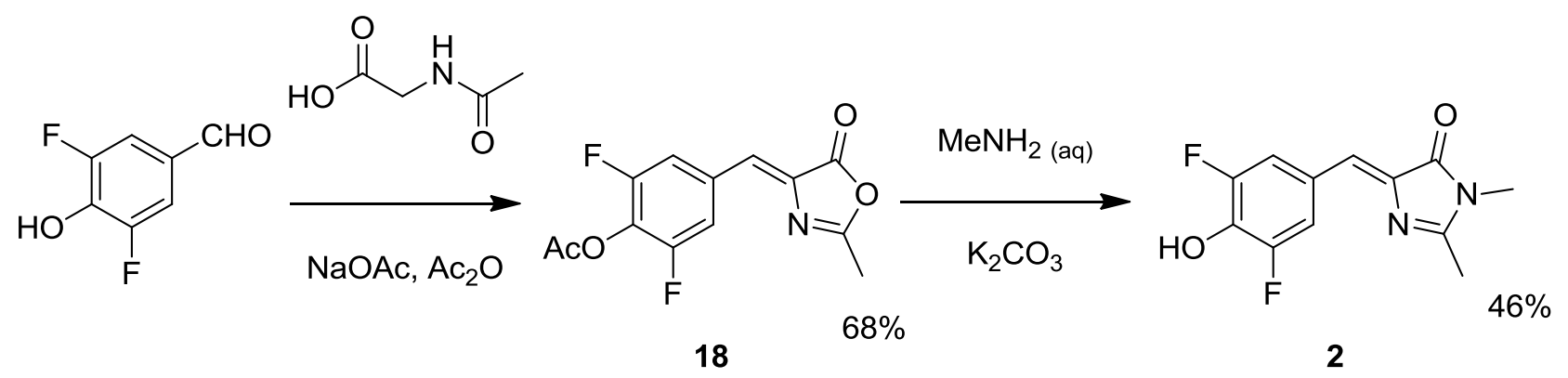

Scheme 6. Synthesis of DFHBI

\section{Conclusion}

In summary, visualization of gene expression via FRET is a viable method, and can now be accomplished in real time. We successfully modified the synthesis of PDC-Gly ligand to a more reliable, consistent approach that was comparable in yield to the previously established method. Further, we successfully coupled Cy3 and Cy5 dyes to PDC-Gly to generate a FRET pair for imaging. During biological testing, Cy3-PDC-Gly and Cy5-PDC-Gly successfully bound to the cell synthesized aptamer chain, allowing for the visualization of FRET. To further show the utility of our biological system, aminoglycoside FRET pairs 14 and 15, 16 and 17 were also synthesized. They too showed successful binding to their respective aptamers and thus a FRET signal was also recorded. Our results firmly establish the viability for imaging gene expression in real time.

In comparison to previously established cellular visualization techniques utilizing green fluorescent protein, our system proved to more sensitive. When tested in our system, DFHBI did not increase in fluorescence when the cells were induced to synthesize Spinach RNA strands. Therefore, DFHBI was not sensitive enough to detect the much less abundant mRNA strands made within the cell. We believe our method of in vivo cellular imaging offers a real time solution to cellular expression via FRET imaging and has been shown to be flexible and robust 
for multiple dye-pair ligands. Further, our results will allow for gene tracking when rapid changes occur within cells and offer greater sensitivity over previous cellular imaging systems.

\section{Experimental Section}

All NMR spectra were obtained on a Varian VXR spectrometer, operating at 300 or $400 \mathrm{MHz}$

for ${ }^{1} \mathrm{H}$ NMR and 75 or $100 \mathrm{MHz}$ for ${ }^{13} \mathrm{C}$ NMR instrument. Chemical shifts in $\mathrm{CDCl}_{3}$ were reported downfield from TMS $(=0 \mathrm{ppm})$ for ${ }^{1} \mathrm{H}$ NMR. Chemical shifts in $\mathrm{CD}_{3} \mathrm{OD}$ were reported relative to the solvent signal $\left[\mathrm{CD}_{3} \mathrm{OD}(=3.31 \mathrm{ppm})\right]$ for ${ }^{1} \mathrm{H} \mathrm{NMR}$. For ${ }^{13} \mathrm{C}$ NMR, chemical shifts were reported relative to the solvent signal $\left[\mathrm{CDCl}_{3}(77.15 \mathrm{ppm})\right]$. All reactions were carried out under argon unless otherwise noted. Thin-layer chromatography was performed using commercially available 250 micron silica gel plates (Analtech). Preparative thin-layer chromatography was performed using commercially available 1000 micron silica gel plates (Analtech). Visualization of TLC plates was effected with short wavelength ultra violet light (254 nm). High resolution mass spectra were recorded on an Agilent 6540 QTOF using EI, ESI, or ACPI. Water was the preferred solvent; methanol was avoided due to poor solubility of aminoglycosides in methanol. All reagents were used directly as obtained commercially unless otherwise noted. Sulfonated cyanine dyes were purchased from Fisher Scientific; non-sulfonated cyanine dyes, Lumiprobe.

\section{General procedures for the synthesis of dye labeled ligands:}

Method A: ${ }^{11}$ In a $20 \mathrm{~mL}$ vial equipped with a stirring bar, Cyanine dye NHS ester (1 equiv.), PDC-Gly'HCl (1 equiv.) and N,N-diisopropylethylamine (20 equiv.) were taken in DMSO (1.0 $\mathrm{mL}$ ) and stirred at room temperature under argon atmosphere in dark for 12 hours. The vial was 
wrapped in aluminum foil to ensure complete darkness while reacting. After the completion of reaction, the reaction mixture was purified by preparative TLC using $5 \% \mathrm{MeOH}: \mathrm{CH}_{2} \mathrm{Cl}_{2}$.

Method B: ${ }^{20}$ In a $20 \mathrm{~mL}$ vial equipped with a stirring bar, cyanine dye NHS ester (1 equiv.) and aminoglycoside (1.1 equiv.) were taken in a $0.1 \mathrm{M}$ solution of sodium bicarbonate in water (1.0 $\mathrm{mL}$ ) and stirred at room temperature in the dark for 12 hours. The vial was wrapped in aluminum foil to ensure complete darkness while reacting. After reaction completion, the mixture was purified by column chromatography using a 5:4:1 mixture of $\mathrm{CH}_{2} \mathrm{Cl}_{2}: \mathrm{MeOH}: \mathrm{NH}_{4} \mathrm{OH}$ through a Pasteur pipette filled with silica gel to give the product.

Method C: ${ }^{20}$ In a $20 \mathrm{~mL}$ vial equipped with a stirring bar, cyanine dye NHS ester (1 equiv.), aminoglycoside (1.1 equiv.), and $N, N$-diisopropylethylamine (20 equiv.) were taken in dry DMSO (1.0 mL) and stirred at room temperature under argon atmosphere in the dark for 12 hours. The vial was wrapped in aluminum foil to ensure complete darkness while reacting. After reaction completion, the mixture was purified by preparative TLC using a 5:4:1 mixture of $\mathrm{CH}_{2} \mathrm{Cl}_{2}: \mathrm{MeOH}: \mathrm{NH}_{4} \mathrm{OH}$ to give the product.

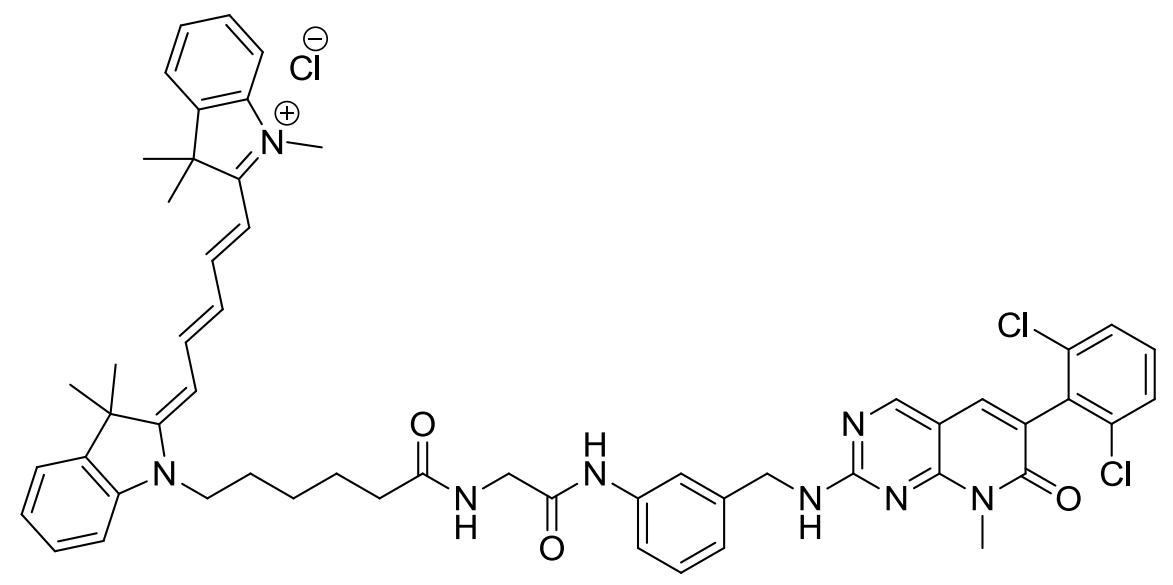

Cy5-PDC-Gly 12: Prepared using method A. HRMS ESI (m/z): calcd for $\mathrm{C}_{55} \mathrm{H}_{57} \mathrm{Cl}_{2} \mathrm{~N}_{8} \mathrm{O}_{3}^{+}\left[\mathrm{M}^{+}\right]$, 947.3925; found, 947.3924. 


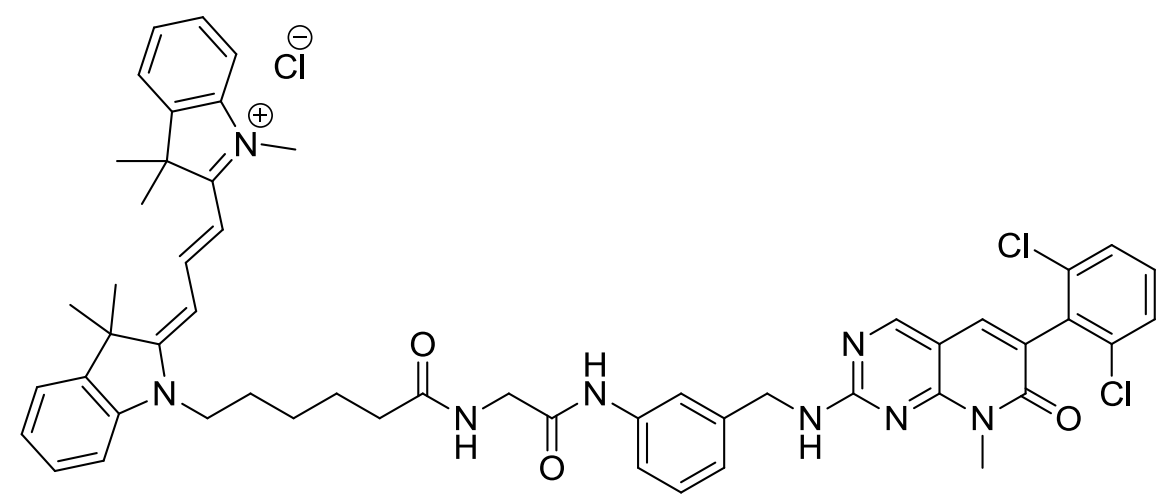

Cy3-PDC-Gly 13: Prepared using method A. HRMS ESI (m/z): calcd for $\mathrm{C}_{53} \mathrm{H}_{55} \mathrm{Cl}_{2} \mathrm{~N}_{8} \mathrm{O}_{3}^{+}\left[\mathrm{M}^{+}\right]$, 921.3769; found, 947.3769.

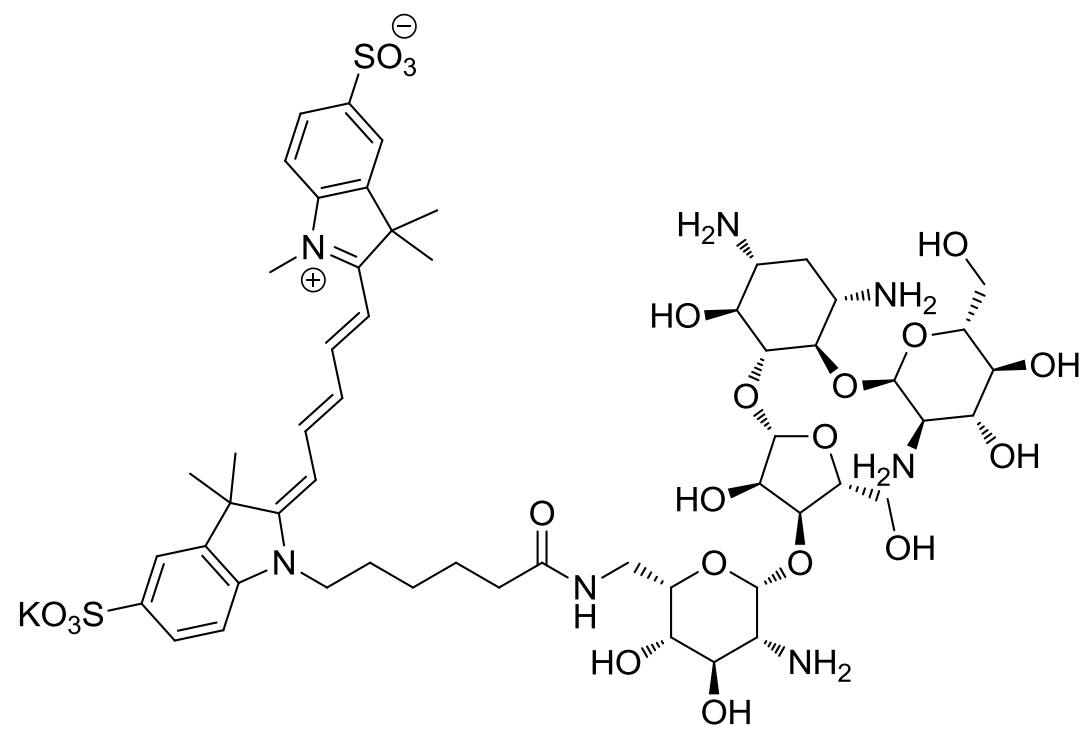

Cy5-Paromomycin 14: Prepared using method B. HRMS ESI (m/z): calcd for $\mathrm{C}_{55} \mathrm{H}_{80} \mathrm{~N}_{7} \mathrm{O}_{21} \mathrm{~S}_{2}{ }^{-}\left[\mathrm{M}^{-}\right], 1238.4854$; found, 1238.4912. 


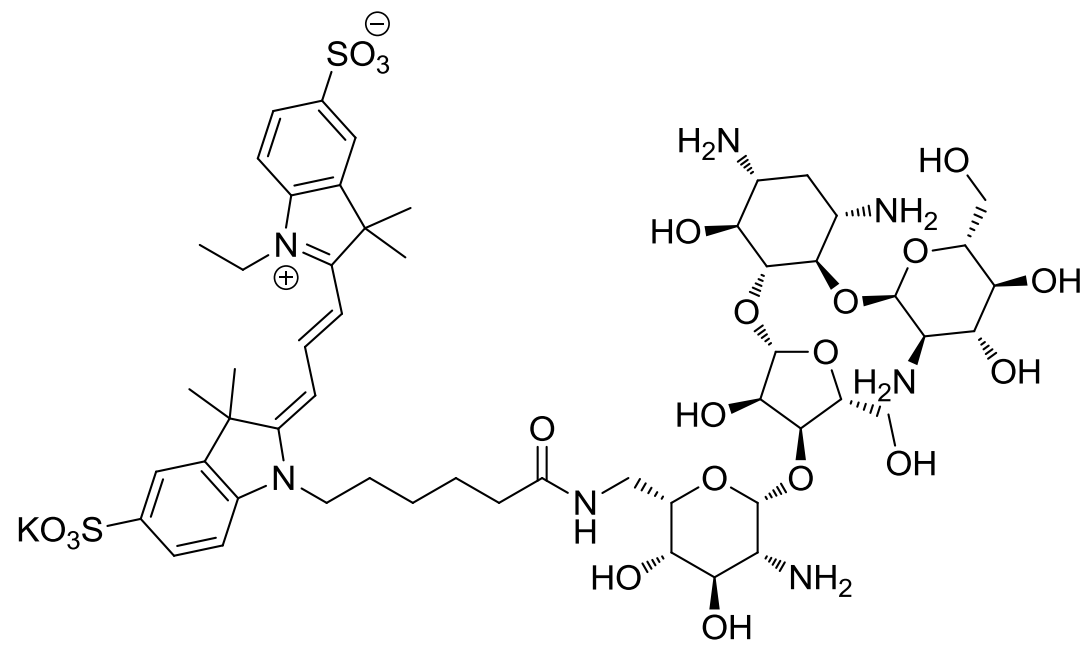

Cy3-Paromomycin 15: Prepared using method B. HRMS ESI (m/z): calcd for $\left.\mathrm{C}_{54} \mathrm{H}_{80} \mathrm{~N}_{7} \mathrm{O}_{21} \mathrm{~S}_{2}{ }^{-}{ }^{-} \mathrm{M}^{-}\right]$, 1226.4854; found, 1226.4839 .

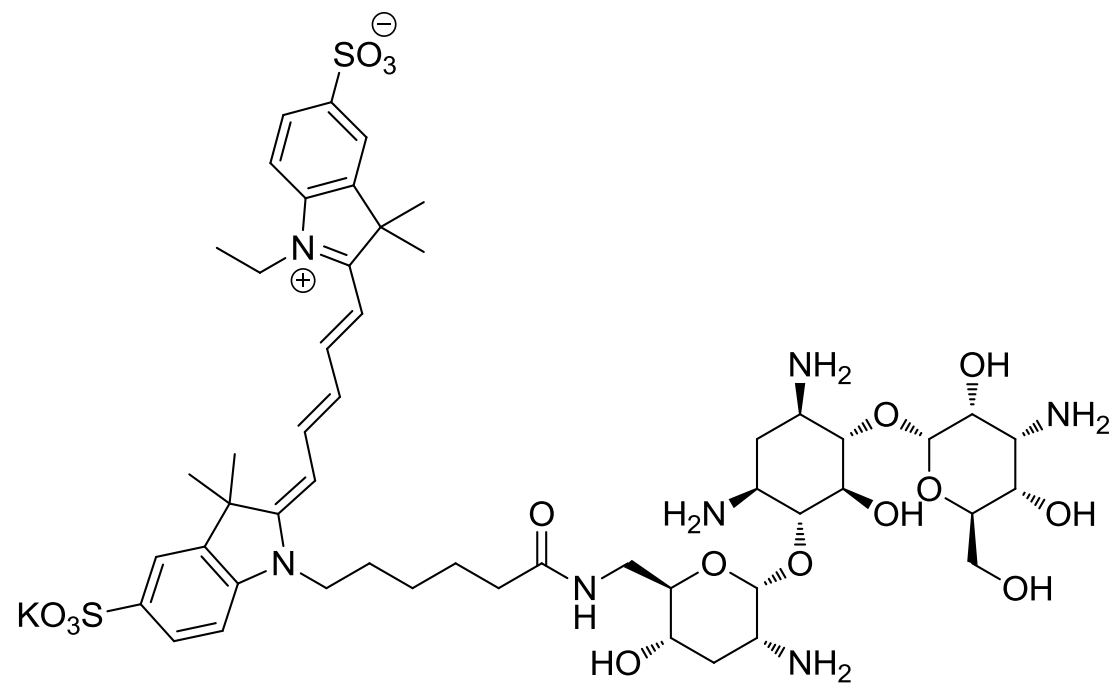

Cy5-Tobramycin 16: Prepared using method C. HRMS ESI (m/z): calcd for $\left.\mathrm{C}_{51} \mathrm{H}_{74} \mathrm{~N}_{7} \mathrm{O}_{16} \mathrm{~S}_{2}{ }^{-}{ }^{-} \mathrm{M}^{-}\right], 1104.4633$; found, 1104.4606. 


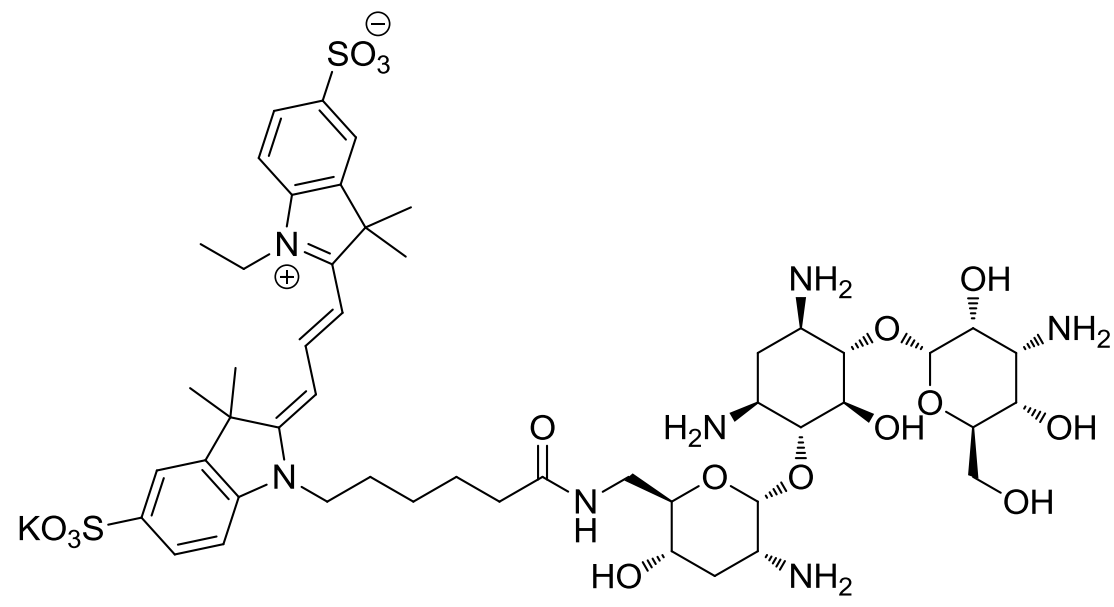

Cy3-Tobramycin 17: Prepared using method C. HRMS ESI (m/z): calcd for $\mathrm{C}_{49} \mathrm{H}_{72} \mathrm{~N}_{7} \mathrm{O}_{16} \mathrm{~S}_{2}{ }^{-}\left[\mathrm{M}^{-}\right], 1078.4477$; found, 1078.4447.<smiles>CCOC(=O)c1cnc(SC)nc1NC</smiles>

Ethyl 4-(methylamino)-2-(methylthio)pyrimidine-5-carboxylate $4:^{21}$ To a solution of $3(4.03 \mathrm{~g}, 17 \mathrm{mmol})$ in THF $(55 \mathrm{~mL})$ was added triethylamine $(7.2 \mathrm{~mL}, 52 \mathrm{mmol})$ followed by a $40 \%$ solution of methylamine in water $(6.6 \mathrm{~mL}, 76 \mathrm{mmol})$. The reaction was stirred overnight at room temperature. The solvent was removed under vacuum, and the solid was suspended in ethyl acetate. The organic layer was washed with saturated $\mathrm{NaHCO}_{3}$, brine, and dried over anhydrous $\mathrm{MgSO}_{4}$. The slurry was filtered and the solvent was removed under vacuum. The resulting residue was triturated with hexanes. Off-white solid $(2.06 \mathrm{~g}, 52 \%) .{ }^{1} \mathrm{H}$ NMR (300 $\left.\mathrm{MHz}, \mathrm{CDCl}_{3}\right): 8.61(\mathrm{~s}, 1 \mathrm{H}), 8.19$ (br s, 1H), $4.32(\mathrm{q}, \mathrm{J}=7.2 \mathrm{~Hz}, 2 \mathrm{H}), 3.08(\mathrm{~d}, \mathrm{~J}=5.1 \mathrm{~Hz}, 3 \mathrm{H})$, $2.55(\mathrm{~s}, 3 \mathrm{H}), 1.37(\mathrm{t}, \mathrm{J}=7.2 \mathrm{~Hz}, 3 \mathrm{H})$. 
<smiles>CNc1nc(SC)ncc1CO</smiles>

\section{(4-(Methylamino)-2-(methylthio)pyrimidin-5-yl)methanol 5: ${ }^{21}$ To a solution of}

lithium aluminum hydride $(325 \mathrm{mg}, 8.6 \mathrm{mmol})$ in dry $\mathrm{THF}(20 \mathrm{~mL})$ at $0{ }^{\circ} \mathrm{C}$ was added a solution of 4 (1.3 g, $5.7 \mathrm{mmol})$ in THF (30 mL) slowly. After gas evolution, the reaction was warmed to room temperature and stirred overnight. The grey reaction suspension was quenched with water $(0.3 \mathrm{~mL}), 10 \% \mathrm{NaOH}(0.3 \mathrm{~mL})$, and water $(1.0 \mathrm{~mL})$ sequentially. The slurry was quickly filtered through Celite ${ }^{\circledR}$ and the filter pad was washed with THF. The solvent was removed under vacuum, and the resulting residue was triturated with a $25 \%$ solution of ethyl acetate in hexanes. Yellow/white solid (726 mg, 69\%). ${ }^{1} \mathrm{H}$ NMR (300 MHz, $\mathrm{CDCl}_{3}$ ): 7.72 (s, 1H), 5.65 (br s, 1H), $4.52(\mathrm{~s}, 2 \mathrm{H}), 3.04(\mathrm{~d}, \mathrm{~J}=3.1 \mathrm{~Hz}, 3 \mathrm{H}), 2.53(\mathrm{~s}, 3 \mathrm{H})$.<smiles>CNc1nc(SC)ncc1C=O</smiles>

4-(Methylamino)-2-(methylthio)pyrimidine-5-carbaldehyde $6:^{21}$ A mixture of 5 (726 $\mathrm{mg}, 3.9 \mathrm{mmol})$ and $85 \%$ activated manganese(II) dioxide $(3.8 \mathrm{~g}, 37 \mathrm{mmol})$ in dry chloroform (50 $\mathrm{mL}$ ) was stirred overnight at room temperature. The reaction slurry was filtered through Celite ${ }^{\circledR}$ and the filter pad was washed with chloroform. The solvent was removed under vacuum, and the resulting residue was triturated with hexanes. Yellow/white solid (523 mg, 73\%). ${ }^{1} \mathrm{H}$ NMR (300 $\mathrm{MHz}, \mathrm{CDCl}_{3}$ ): 9.70 (s, 1H), 8.59 (br s, 1H), 8.30 (s, 1H) 3.12 (d, J = 6.2 Hz, 3H), 2.57 (s, 3H). 


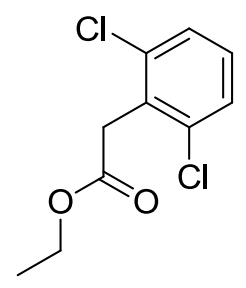

Ethyl 2-(2,6-dichlorophenyl)acetate 7: To a solution of 2-(2,6-dichlorophenyl)acetic acid $(2.04 \mathrm{~g}, 9.9 \mathrm{mmol})$ in ethanol $(20 \mathrm{~mL})$ was added concentrated sulfuric acid $(0.5 \mathrm{~mL}, 9.0$ mmol) dropwise with stirring. The reaction was heated to reflux overnight. After cooling to room temperature, the solvent was removed under vacuum. The oil was dissolved in ethyl acetate and washed twice with saturated $\mathrm{NaHCO}_{3}$, dried over anhydrous $\mathrm{MgSO}_{4}$ and filtered. The solvent was removed under vacuum. No further purification was needed. Yellow oil (2.31 g, 100\%). ${ }^{1} \mathrm{H}$ NMR (300 MHz, $\left.\mathrm{CDCl}_{3}\right): 7.32(\mathrm{~d}, \mathrm{~J}=8.2 \mathrm{~Hz}, 2 \mathrm{H}), 7.16(\mathrm{t}, \mathrm{J}=7.5 \mathrm{~Hz}, 1 \mathrm{H}), 4.19$ $(\mathrm{q}, \mathrm{J}=7.5 \mathrm{~Hz}, 2 \mathrm{H}), 4.01(\mathrm{~s}, 2 \mathrm{H}), 1.26(\mathrm{t}, \mathrm{J}=6.5 \mathrm{~Hz}, 3 \mathrm{H})$.<smiles>CSc1ncc2cc(-c3c(Cl)cccc3Cl)c(=O)n(C)c2n1</smiles>

\section{6-(2,6-Dichlorophenyl)-8-methyl-2-(methylthio)pyrido[2,3-d]pyrimidin-7(8H)-one}

8: ${ }^{10,22}$ To a solution of 6 (357 mg, $\left.1.95 \mathrm{mmol}\right)$ and 7 (572 $\left.\mathrm{mg}, 2.45 \mathrm{mmol}\right)$ in N,Ndimethylacetamide $(8.9 \mathrm{~mL})$ was added $40 \%$ potassium fluoride on aluminum oxide $(2.26 \mathrm{~g}, 16$ $\mathrm{mmol}$ ) in $0.5 \mathrm{~g}$ portions every 30 minutes. The resulting brown reaction suspension was stirred overnight at room temperature. The aluminum oxide was filtered through Celite ${ }^{\circledR}$ and the filter pad was washed with dichloromethane. The filtrate was concentrated under vacuum utilizing a toluene azeotrope. The brown residue was recrystallized from DMF. Orange solid (209 mg, 
31\%). ${ }^{1} \mathrm{H} \mathrm{NMR}\left(400 \mathrm{MHz}, \mathrm{CDCl}_{3}\right): 8.67$ (s, 1H), $7.61(\mathrm{~s}, 1 \mathrm{H}), 7.41-7.44(\mathrm{~m}, 2 \mathrm{H}), 7.26-7.32$ (m, 1H), $3.84(\mathrm{~s}, 3 \mathrm{H}), 2.67(\mathrm{~s}, 3 \mathrm{H})$.<smiles>COc1ncc2cc(-c3c(Cl)cccc3Cl)c(=O)n(C)c2n1</smiles>

\section{6-(2,6-Dichlorophenyl)-8-methyl-2-(methylsulfonyl)pyrido[2,3-d]pyrimidin-7(8H)-}

one 9: ${ }^{10}$ To a solution of $8(175 \mathrm{mg}, 0.5 \mathrm{mmol})$ in chloroform $(20 \mathrm{~mL})$ was added $75 \%$ metachloroperbenzoic acid $(250 \mathrm{mg}, 1.1 \mathrm{mmol})$ at room temperature with stirring. The resulting yellow solution was stirred overnight at room temperature. The reaction was then quenched with DMSO (2 mL) and stirred for 15 minutes. The reaction was diluted with dichloromethane, and washed with saturated $\mathrm{NaHCO}_{3}$, water, and brine respectively. The organic layer was dried over anhydrous $\mathrm{MgSO}_{4}$, filtered, and concentrated under vacuum. Yellow solid (228 mg, 100\%). ${ }^{1} \mathrm{H}$ NMR (400 MHz, $\left.\mathrm{CDCl}_{3}\right)$ : $9.03(\mathrm{~s}, 1 \mathrm{H}), 7.78(\mathrm{~s}, 1 \mathrm{H}), 7.44-7.47(\mathrm{~m}, 2 \mathrm{H}), 7.32-7.38$ (m, 1H), 3.92 $(\mathrm{s}, 3 \mathrm{H}), 3.45(\mathrm{~s}, 3 \mathrm{H})$.<smiles>Cn1c(=O)c(-c2c(Cl)cccc2Cl)cc2cnc(NCc3cccc(N)c3)nc21</smiles>

Amino-PDC 1: ${ }^{10}$ To a solution of 9 (39 mg, $\left.0.1 \mathrm{mmol}\right)$ in dry DMF (3 mL) was added 3-aminobenzylamine from Sigma-Aldrich ${ }^{\circledR}(26 \mathrm{mg}, 0.22 \mathrm{mmol})$ at room temperature with stirring. The reaction was heated overnight at $150{ }^{\circ} \mathrm{C}$. Heating to reflux results in two products: PDC- $\mathrm{NH}_{2}$ and formylated PDC-NH 2 . After cooling to room temperature, the reaction was quenched with water and extracted three times with ethyl acetate. The combined organic layers 
were washed three times with water, brine, and dried over anhydrous $\mathrm{MgSO}_{4}$. The slurry was filtered and concentrated under vacuum. The residue was purified by preparative TLC using $70 \%$ ethyl acetate:hexanes. Orange solid (35 mg, 82\%). ${ }^{1} \mathrm{H}$ NMR (300 $\left.\mathrm{MHz}, \mathrm{CDCl}_{3}\right): 8.35$ (br s, 2H), 7.39-7.45 (m, 3H), 7.22-7.28 (m, 2H), $7.14(\mathrm{t}, \mathrm{J}=7.8 \mathrm{~Hz}, 1 \mathrm{H}), 6.60-6.79(\mathrm{~m}, 3 \mathrm{H}), 6.29$ (br s, $1 \mathrm{H}), 4.65(\mathrm{~d}, \mathrm{~J}=6.0 \mathrm{~Hz}, 2 \mathrm{H}), 3.72(\mathrm{~s}, 3 \mathrm{H})$. HRMS ESI $(\mathrm{m} / \mathrm{z})$ : calcd for $\mathrm{C}_{21} \mathrm{H}_{18} \mathrm{Cl}_{2} \mathrm{~N}_{5} \mathrm{O}\left[\mathrm{M}^{+}\right.$ $+\mathrm{H}], 426.0883$; found, 426.0892 .<smiles>Cn1c(=O)c(-c2c(Cl)cccc2Cl)cc2cnc(NCc3cccc(NC(=O)CNC(=O)OC(C)(C)C)c3)nc21</smiles>

Boc-PDC-Gly 11: ${ }^{19}$ To a solution of 1 (8.2 mg, $\left.0.02 \mathrm{mmol}\right), \mathrm{N}-($ tert-butoxycarbonyl) glycine $(4.8 \mathrm{mg}, 0.03 \mathrm{mmol})$, and N-hydroxybenzotriazole $(2.9 \mathrm{mg}, 0.02 \mathrm{mmol})$ in dry THF (1 $\mathrm{mL}$ ) was added N,N'-dicyclohexylcarbodiimide (4.5 mg, $0.02 \mathrm{mmol})$. The yellow reaction solution was stirred overnight at room temperature. The formed white precipitate, N,N'dicyclohexylurea, was filtered off. The filter pad was washed with ethyl acetate, and the solvent was removed under vacuum. The solid residue was purified by preparative TLC using $70 \%$ ethyl acetate:hexanes. Orange solid (7.4 mg, 66\%). ${ }^{1} \mathrm{H} \mathrm{NMR}\left(300 \mathrm{MHz}, \mathrm{CDCl}_{3}\right):$ 7.16-7.38 (m, 9H), $4.72(\mathrm{~s}, 2 \mathrm{H}), 3.91(\mathrm{~s}, 2 \mathrm{H}), 3.70(\mathrm{~s}, 3 \mathrm{H}), 1.46(\mathrm{~s}, 9 \mathrm{H})$.<smiles>Cn1c(=O)c(-c2c(Cl)cccc2Cl)cc2cnc(NCc3cccc(NC(=O)CN)c3)nc21</smiles> 
PDC-Gly'HCl 10: ${ }^{19}$ To a solution of $11(7.4 \mathrm{mg}, 0.01 \mathrm{mmol})$ in ethyl acetate $(5 \mathrm{~mL})$ was added $3 \mathrm{~N}$ hydrochloric acid $(1 \mathrm{~mL})$. The suspension was stirred overnight at room temperature. The product was concentrated under vacuum. Thin-layer chromatography showed no starting material; the yellow solid was taken on as crude to the dye labeling steps.<smiles>CC(=O)Oc1c(F)cc(/C=C2\N=C(C)OC2=O)cc1F</smiles>

(Z)-2,6-difluoro-4-((2-methyl-5-oxooxazol-4(5H)-ylidene)methyl)phenyl acetate 18: ${ }^{15}$ 3,5-Difluoro-4-hydroxybenzaldehyde (260.6 mg, $1.64 \mathrm{mmol})$, N-acetylglycine (194.1 mg, 1.66 $\mathrm{mmol})$, anhydrous sodium acetate $(135.8 \mathrm{mg}, 1.66 \mathrm{mmol})$, and acetic anhydride $(0.61 \mathrm{~mL}, 6.45$ mmol) were combined and stirred at $110{ }^{\circ} \mathrm{C}$ for 2 hours. After cooling to room temperature, cold ethanol $(6 \mathrm{~mL})$ was added and the slurry was cooled to $4{ }^{\circ} \mathrm{C}$ and set overnight. The resulting solid was filtered and washed with a small amount of cold ethanol, hot water, and hexanes. Yellow solid (303.6 mg, 68\%). ${ }^{1} \mathrm{H}$ NMR (300 MHz, $\left.\mathrm{CDCl}_{3}\right): 7.77$ (d, J = 8.7 Hz, 2H), 6.96 (s, 1H), $2.43(\mathrm{~s}, 3 \mathrm{H}), 2.40(\mathrm{~s}, 3 \mathrm{H})$.<smiles>CC1=N/C(=C\c2cc(F)c(O)c(F)c2)C(=O)N1C</smiles>

\section{(Z)-4-(3,5-difluoro-4-hydroxybenzylidene)-1,2-dimethyl-1H-imidazol-5(4H)-one}

(DFHBI) 2: ${ }^{15}$ To a solution of $18(155 \mathrm{mg}, 0.55 \mathrm{mmol})$ in ethanol $(2.5 \mathrm{~mL})$ was added a $40 \%$ aqueous solution of methylamine in water $(0.16 \mathrm{~mL}, 1.8 \mathrm{mmol})$ followed by potassium carbonate (110.0 mg, $0.80 \mathrm{mmol}$ ). The order of addition was critical for a successful reaction. The slurry 
was heated to reflux for 3 hours then cooled to room temperature. The formed brown precipitate was filtered and washed with a small amount of cold ethanol. The precipitate was dissolved in a 1:1 mixture of ethyl acetate and $500 \mathrm{mM}$ sodium acetate buffer $(\mathrm{pH} 3.0)$. The organic layer was removed and dried over anhydrous $\mathrm{MgSO}_{4}$. After filtration, the solvent was removed in vacuo. The yellow solid was triturated with diethyl ether. Yellow solid, stored in the dark $(63.2 \mathrm{mg}$, 46\%). ${ }^{1} \mathrm{H} \mathrm{NMR}\left(300 \mathrm{MHz}, \mathrm{CD}_{3} \mathrm{OD}\right): 7.77$ (dd, $\left.J=8.1,1.8 \mathrm{~Hz}, 2 \mathrm{H}\right), 6.90(\mathrm{~s}, 1 \mathrm{H}), 3.18$ (s, 3H),

2.40 (s, 3H). HRMS ESI (m/z): calcd for $\mathrm{C}_{12} \mathrm{H}_{11} \mathrm{~F}_{2} \mathrm{~N}_{2} \mathrm{O}_{2}^{+}\left[\mathrm{M}^{+}+\mathrm{H}\right]$, 253.0789; found, 253.0783.

\section{References}

1. Klein, S.; Levitzki, A. Adv. Cancer Res. 2007, 97, 295-319.

2. (a) Levitzki, A. Curr. Opin. Cell Biol. 1996, 8, 239-244. (b) Bridges, A. J. Expert Opin. Ther. Pat. 1995, 5, 1245-1257. (c) Spada, A. P.; Myers, M. R. Expert Opin. Ther. Pat. 1995, 5, 805-817. (d) Traxler, P.; Lydon, N. Drugs of the Future 1995, 20, 1261-1274.

3. Schindler, T.; Bornmann, W.; Pellicena, P.; Miller, W. T.; Clarkson, B.; Kuriyan, J. Science 2000, 289, 1938-1942.

4. Ramirez, P.; DiPersio, J. F. Oncologist, 2008, 13, 424.

5. Moasser, M. M.; Srethapakdi, M.; Sachar, K. S.; Kraker, A. J.; Rosen, N. Cancer Res. 1999, 59, 6145-6152.

6. Hamby, J. M.; Connolly, C. J.; Schroeder, M. C.; Winters, R. T.; Showalter, H. D.; Panek, R. L.; Major, T. C.; Olsewski, B.; Ryan, M. J.; Dahring, T.; Lu, G. H.; Keiser, J.; Amar, A.; Shen, C.; Kraker, A. J.; Slintak, V.; Nelsen, J. M.; Fry, D. W.; Bradford, L.; Hallak, H.; Doherty, A. M. J. Med. Chem. 1997, 40, 2296-2303.

7. Nagar, B.; Bornmann, W. G.; Pellicena, P.; Schindler, T.; Veach, D. R.; Miller, W. T.; Clarkson, B.; Kuriyan, J. Cancer Res. 2002, 62, 4236-4243.

8. Wisniewski, D.; Lambek, C. L.; Liu, C.; Strife, A.; Veach, D. R.; Nagar, B.; Young, M. A.; Schindler, T.; Bornmann, W. G.; Bertino, J. R.; Kuriyan, J.; Clarkson, B. Cancer Res. 2002, 62, 4244-4255.

9. Wissing, J.; Godl, K.; Brehmer, D.; Blencke, S.; Weber, M.; Habenberger, P.; SteinGerlach, M.; Missio, A.; Cotton, M.; Müller, S.; Daub, H. Mol. Cell. Proteomics 2004, 3, 1181-1193. 
10. Kraus, G. A.; Gupta, V. Mokhtarian, M.; Mehanovic, S.; Nilsen-Hamilton, M. Bioorg. Med. Chem. 2010, 18, 6316-6321.

11. Shin, I.; Ray, J.; Gupta, V.; Ilgu, M.; Haarberg, H. E.; Beasley, J.; Bendickson, L.; Mehanovic, S.; Kraus, G. A.; Nilsen-Hamilton, M. Nat. Biotechnol. submitted.

12. Endoh, T.; Mie, M.; Kobatake, E.; J. Biotechnol. 2008, 133, 413-417.

13. (a) Bertrand, E.; Chartrand, P.; Schaefer, M.; Shenoy, S. M.; Singer, R. H.; Long, R. M. Mol Cell 1998, 2, 437-445. (b) Larson, D. R.; Zenklusen, D.; Wu, B.; Chao, J. A.; Singer, R. H. Science 2011, 332, 475-478.

14. Chudakov, D. M.; Matz, M. V.; Lukyanov, S.; Lukyanov, K .A. Physiol. Rev. 2010, 90, 1103-1163.

15. Paige, J. S.; Wu, K. Y.; Jaffrey, S. R. Science, 2011, 333, 642-646.

16. Helms, Volkhard Fluorescence Resonance Energy Transfer Principles of Computational Cell Biology Weinheim: Wiley-VCH, 2008, 202.

17. Clegg, R. M. The History of FRET Reviews in Fluorescence Springer, 2006, 1.

18. Jares-Erijman, E. A.; Jovin, T. M. Nat. Biotechnol. 2003, 21, 1387-1395.

19. Gao, C.; Liu, F.; Luan, X.; Tan, C.; Liu, H.; Xie, Y; Jin, Y.; Jiang, Y. Bioorg. Med. Chem. 2010, 18, 7507-7514.

20. Lumiprobe: Life Science Solutions. NHS Ester Labeling of Amino Biomolecules. http://www.lumiprobe.com/protocols/nhs-ester-labeling (accessed Mar 22, 2013).

21. Klutchko, S. R.; Hamby, J. M.; Boschelli, D. H.; Wu, Z.; Kraker, A. J.; Amar, A. M.; Hartl, B. G.; Shen, C.; Klohs, W. D.; Steinkampf, R. W.; Driscoll, D. L.; Nelson, J. M.; Elliott, W. L.; Roberts, B. J.; Stoner, C. L.; Vincent, P. W.; Dykes, D. J.; Panek, R. L.; Lu, G. H.; Major, T. C.; Dahring, T. K.; Hallak, H.; Bradford, L. A.; Showalter, H. D. H.; Doherty, A. M. J. Med. Chem. 1998, 41, 3276-3292.

22. Blass, B. E.; Coburn, K.; Fairweather, N.; Sabat, M.; West, L. Tetrahedron Lett. 2006, 47, 3177-3180. 


\section{GENERAL CONCLUSIONS}

The synthesis and study of some novel heteroaromatic compounds have been shown in this dissertation. Chapter 1 describes recent advances in Kraus indole synthesis technology. First, the effect of sulfide groups was analyzed in comparison to phosphonium, arylsulfone, and nitrile groups in the six-electron ring closure step. Similar to the products from phosphonium and arylsulfone starting materials, sulfides eliminated and resulted in 2-substituted indoles. With this result, we also made 2,3-disubstituted indoles from 2-(bis(methylthio)methyl)aniline. Extending the science even further, we proceeded to synthesize indolo[2,1-a]isoquinolines under the same Kraus indole conditions with 2-alkynyl benzaldehydes. We also discovered the scope and limitations of this chemistry by synthesizing novel 2-(2-alkynylphenyl)-1H-indoles. The conversion of readily available 2 -halobenzaldehydes into indolo[2,1-a]isoquinolines in two operations represents a very direct entry to this class of molecules. Specifically, these indolo[2,1-a]isoquinolines can be useful in the synthesis of (S)-(-)-cryptaustoline and (S)-(-)cryptowoline.

Chapter 2 describes recent advances in renewable chemistry. Specifically, commodity maleic acid has been successfully synthesized from furfural, a possible product from pentoses, via a two-stage oxidation. The first step involves green singlet oxygen oxidation, while the second step utilizes ruthenium(III) and a reoxidant. From hexoses we successfully made the first reported furan-based ionic liquids. The ionic liquids were synthesized from known platform chemical CMF and tertiary amines. This synthesis represents a direct entry in two transformations to potentially useful specialty chemicals.

Chapter 3 describes the synthesis and optimization of PDC-Gly, a ligand used in molecular imaging. FRET pairs were synthesized for PDC-Gly, tobramycin, and paromomycin 
with Cy3 and Cy5. These molecules showed FRET when irradiated, indicating successful gene promotion in vivo resulting in mRNA aptamer synthesis and ligand binding. To test the sensitivity of our system, DFHBI was also synthesized and intercalated into cells. Unlike the FRET pair ligands, no increase in fluorescence was noticed, indicating poor sensitivity of DFHBI toward mRNA. 


\section{ACKNOWLEDGEMENTS}

First of all, I would like to give my greatest appreciation to my major professor, Dr. George A. Kraus, who lead me throughout my graduate career. His mentoring in research, experimental expertise, and supreme understanding of the chemical literature benefited me greatly. Myriad thanks to Dr. Kraus for guidance on my research and exceptional support.

Secondly, I would like to thank my graduate committee members; Dr. William Jenks, Dr. Raj Raman, Dr. Klaus Schmidt-Rohr, and Dr. Yan Zhao, for their support, time, and patience as I worked toward my Ph.D. degree.

Thirdly, I would like to thank Dr. Kraus' group members, past and present, for providing a friendly and professional work environment. Their guidance, knowledge, and support were greatly beneficial.

I would like to leave my final acknowledgement for my family. I thank my parents for their ever present support and prayers. They have devoted a lifetime of commitment toward me and constantly encouraged me in my educational efforts. I also thank my beautiful wife Cheryl, for her deep and loving patience, encouragement, and prayers. Together we have tackled monumental goals and events together, and her presence has been felt in all aspects of my life. I would lastly thank my soon-to-be-born baby, for bringing a smile to my face and offering a promise of the future. 